FACTA UNIVERSITATIS (NIŠ)

Ser. Math. Inform. Vol. 35, No 2 (2020), 741-773

https://doi.org/10.22190/FUMI2003741T

\title{
TWO NOTABLE CLASSES OF PROJECTIVE VECTOR FIELDS
}

\author{
Tayebeh Tabatabaeifar, Mehdi Rafie-Rad and Behzad Najafi
}

\begin{abstract}
(C) by University of Niš, Serbia | Creative Commons Licence: CC BY-NC-ND Abstract. Here, we find some necessary conditions for a projective vector field on a Randers metric to preserve the non-Riemannian quantities $\Xi$ and $H$. They are known in the contexts as the $C$-projective and $H$-projective vector fields. We find all projective vector fields of the Funk type metrics on the Euclidean unit ball $\mathbb{B}^{n}(1)$.

Keywords: projective vector field; Randers metric; Funk type metrics; Euclidean unit ball.
\end{abstract}

\section{Introduction}

Beltrami [3] introduced the first examples of projective transformations. The projective Finsler geometry is much complicated than the projective Riemannian geometry. This complexity may impresses several projective properties which used to be proved in projective Riemannian geometry, for example Beltrami's theorem in Riemannian geometry states that the projective (i.e., locally projectively flat). Riemannian metrics are exactly those with constant sectional curvature, while this fails generally within Finsler geometry. This may even affect on the projective algebra (i.e. the lie algebra of the projective vector fields) $\operatorname{proj}(M, F)$ and its subalgebras. To trace this, we may refer to the subalgebras of $\operatorname{proj}(M, F)[7,8]$. The special projective algebra $\operatorname{sproj}(M, F)$ consists of the projective vector fields preserving the Berwald curvature. In [11], it is proved that given any special projective vector field $X$ on a Randers space $(M, F=\alpha+\beta)$ with the navigation data $(h, W)$, either $F$ is isotropic S-curvature or $X$ is a conformal vector field for the Riemannian metric $h$. This result supports a Lichnerowicz-Obata type theorem for the special projective vector fields, see $[4,11]$. It is guessed that some other Lichnerowicz-Obata type theorems may be established for our two notable projective subalgebras, namely, the $\mathrm{C}$-projective and the $\mathrm{H}$-projective algebras. We would like to examine another subalgebras of $\operatorname{proj}(M, F)$ namely, the C-projective algebra and the $H$-invariant projective algebra $\operatorname{cproj}(M, F)$ and $\operatorname{hproj}(M, F)$, respectively. The former is shown here to be characterized by preserving the $\Xi$-curvature and the latter is defined by

Received September 11, 2019; accepted December 30, 2019

2010 Mathematics Subject Classification. Primary 53B40; Secondary 53C60 
preserving the $\mathbf{H}$-curvature. In [9], $C$-projective vector fields are studied. We prove the following infinitesimally stated results:

Theorem 1.1. Let us suppose that $(M, F=\alpha+\beta)$ is a Randers space of dimension $n \geq 2$ and $X$ is a $C$-projective vector field of $(M, F)$. Then, at least one of the statements of (1.1) and (1.2) is held:

$$
\left\{\begin{array}{l}
\text { 1. } \quad F \text { is of isotropic } S \text {-curvature. } \\
\text { or } b^{2} \alpha^{2}\left(\mathcal{L}_{\hat{X}}\left(\alpha^{2}-\beta^{2}\right)-\left(\beta^{2} \mathcal{L}_{\hat{X}} \alpha^{2}-\alpha^{2} \mathcal{L}_{\hat{X}} \beta^{2}\right)=\eta\left(\alpha^{2}-\beta^{2}\right),\right.
\end{array}\right.
$$

where $\eta$ is a polynomial of degree two on TM.

$$
\left\{\begin{array}{l}
\text { 3. } \quad \mathcal{L}_{\hat{X}} \alpha^{2}=\lambda \alpha^{2} \\
\text { or } \\
\text { 4. }\left(8 e_{00} \beta s_{0}-4 e_{00}^{2}+e_{00 ; 0} \beta\right)=\eta \alpha^{2},
\end{array}\right.
$$

where $\lambda \in C^{\infty}(M)$ and $\eta(y)$ is a polynomials of degree two.

In the following, we investigate a bigger class of $C$-projective vector fields, namely, the vector fields which preserve $H$-curvature.

Theorem 1.2. Let us suppose that $(M, F=\alpha+\beta)$ be a compact Randers space of dimension $n \geq 2$ and $X$ is a $H$-projective vector field of $(M, F)$. Then, at least one of the statements of (1.3) and (1.4) is held:

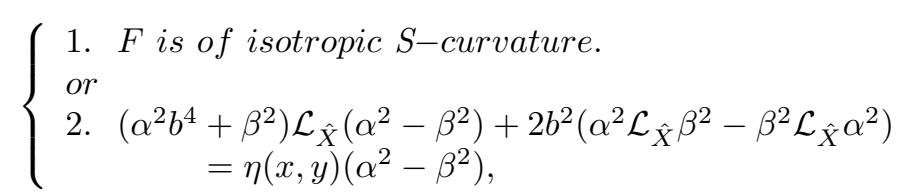

where $\eta$ is a polynomial of degree two on $T M$ and

$$
\begin{cases}\text { 3. } & \mathcal{L}_{\hat{X}} \alpha^{2}=\lambda \alpha^{2} . \\ \text { or } & \\ \text { 4. } & -192 e_{00} \beta s_{0}+96 e_{00}^{2}-19 e_{00 ; 0} \beta=\lambda \alpha^{2}\end{cases}
$$

where $\lambda \in C^{\infty}(M)$ and $\eta(y)$ is a polynomials of degree two.

Here, we study a bigger class of projective transformation, namely, $C$-projective transformation of Randers space. $C$-projective algebra, i. e, the algebra of $C$-projective vector fields on an $n$-dimensional Finsler space is a sub-algebra of projective algebra, and its dimension is $n(n+2)$ at most. Let $F=\alpha+\beta$ be a Randers space. We find the conditions for a vector field to be a $C$-projective vector field. 


\section{Preliminaries}

Let $M$ be a smooth and connected manifold of dimension $n \geq 2$. $T_{x} M$ denotes the tangent space of $M$ at $x$. The tangent bundle of $M$ is the union of tangent spaces $T M:=\bigcup_{x \in M} T_{x} M$. We will denote the elements of $T M$ by $(x, y)$ where $y \in T_{x} M$. Let $T M_{0}=T M \backslash\{0\}$. The natural projection $\pi: T M_{0} \rightarrow M$ is given by $\pi(x, y):=x$. A Finsler metric on $M$ is a function $F: T M \rightarrow[0, \infty)$ with the following properties: (i) $F$ is $C^{\infty}$ on $T M_{0}$, (ii) $F$ is positively 1-homogeneous on the fibers of tangent bundle $T M$, and (iii) the Hessian of $F^{2}$ with elements $g_{i j}(x, y):=\frac{1}{2}\left[F^{2}(x, y)\right]_{y^{i} y^{j}}$ is positive-definite matrix on $T M_{0}$. The pair $(M, F)$ is then called a Finsler space. Throughout this paper, we denote a Riemannian metric by $\alpha=\sqrt{a_{i j}(x) y^{i} y^{j}}$ and a 1 -form by $\beta=b_{i}(x) y^{i}$. A globally defined spray $\mathbf{G}$ is induced by $F$ on $T M_{0}$, which in a standard coordinate $\left(x^{i}, y^{i}\right)$ for $T M_{0}$ is given by $\mathbf{G}=y^{i} \frac{\partial}{\partial x^{i}}-2 G^{i}(x, y) \frac{\partial}{\partial y^{i}}$, where $G^{i}(x, y)$ are local functions on $T M_{0}$ satisfying $G^{i}(x, \lambda y)=\lambda^{2} G^{i}(x, y), \lambda>0$ and given by

$$
G^{i}=\frac{1}{4} g^{i k}\left\{y^{h} F_{x^{h} y^{k}}^{2}-F_{x^{k}}^{2}\right\}
$$

Assume the following conventions:

$$
G_{j}^{i}=\frac{\partial G^{i}}{\partial y^{j}}, \quad G_{j k}^{i}=\frac{\partial G_{j}^{i}}{\partial y^{k}}
$$

The local functions $G_{j}^{i}$ are coefficients of a connection in the pullback bundle $\pi^{*} T M \rightarrow M$ which is called the Berwald connection denoted by $D$. Recall that for instance, the derivatives of a vector field $X$ and a 2 -covariant tensor $T=T_{i j} d x^{i} \otimes d x^{j}$ are given by:

$$
\begin{aligned}
X_{i \mid j} & =\frac{\delta X_{i}}{\delta x^{j}}-X_{r} G_{i j}^{r} \\
T_{i j \mid k} & =\frac{\delta T_{i j}}{\delta x^{k}}-T_{r j} G_{i k}^{r}-T_{i r} G_{j k}^{r}
\end{aligned}
$$

where $\frac{\delta}{\delta x^{k}}=\frac{\partial}{\partial x^{k}}-G_{k}^{i} \frac{\partial}{\partial y^{i}}$. Given a Finsler metric $F$ on an $n$-dimensional manifold $M$, the Busemann-Hausdorff volume form $d V_{F}=\sigma_{F}(x) d x^{1} \cdots d x^{n}$ is defined by

$$
\sigma_{F}(x):=\frac{\operatorname{Vol}\left(\mathbb{B}^{n}(1)\right)}{\operatorname{Vol}\left\{\left(y^{i}\right) \in \mathbb{R}^{n} \mid F\left(\left.y^{i} \frac{\partial}{\partial x^{i}}\right|_{x}\right)<1\right\}} .
$$

Define $\underline{g}=\operatorname{det}\left(g_{i j}(x, y)\right)$ and $\tau(x, y):=\ln \frac{\sqrt{\underline{g}}}{\sigma_{F}(x)}$. Given a vector $y \in T_{x} M$, let $\gamma(t),-\epsilon<t<\epsilon$, denote the geodesic with $\gamma(0)=x$ and $\dot{\gamma}(0)=y$. The function $S(x, y):=\frac{d}{d t}[\tau(\gamma(t), \dot{\gamma}(t))]_{\mid t=0}$ is called the $S$-curvature with respect to BusemannHausdorff volume form. A Finsler space is said to be of isotropic S-curvature if there is a function $c=c(x)$ defined on $M$ such that $S=(n+1) c(x) F$. It is called a Finsler space of constant $S$-curvature when $c$ is constant. Let $(M, \alpha)$ be a Riemannian space and $\beta=b_{i}(x) y^{i}$ be a 1 -form defined on $M$ such that $\|\beta\|_{x}:=$ 
$\sup _{y \in T_{x} M} \beta(y) / \alpha(y)<1$. The Finsler metric $F=\alpha+\beta$ is called a Randers metric on the manifold $M$. Denote the geodesic spray coefficients of $\alpha$ and $F$ by $G_{\alpha}^{i}$ and $G^{i}$, respectively. The Levi-Civita covariant derivative of $\alpha$ is denoted by $\nabla$. Define $\nabla_{j} b_{i}$ by $\left(\nabla_{j} b_{i}\right) \theta^{j}:=d b_{i}-b_{j} \theta_{i}{ }^{j}$, where $\theta^{i}:=d x^{i}$ and $\theta_{i}{ }^{j}:=\tilde{\Gamma}_{i k}^{j} d x^{k}$ denote the Levi-Civita connection forms and $\nabla$ denotes its associated covariant derivation of $\alpha$. Let us put

$$
\begin{aligned}
& r_{i j}:=\frac{1}{2}\left(\nabla_{j} b_{i}+\nabla_{i} b_{j}\right), s_{i j}:=\frac{1}{2}\left(\nabla_{j} b_{i}-\nabla_{i} b_{j}\right), \\
& s^{i}{ }_{j}:=a^{i h} s_{h j}, s_{j}:=b_{i} s^{i}{ }_{j}, e_{i j}:=r_{i j}+b_{i} s_{j}+b_{j} s_{i} .
\end{aligned}
$$

Then $G^{i}$ are given by

$$
G^{i}=G_{\alpha}^{i}+\left(\frac{e_{00}}{2 F}-s_{0}\right) y^{i}+\alpha s_{0}^{i},
$$

where $e_{00}:=e_{i j} y^{i} y^{j}, s_{0}:=s_{i} y^{i}, s_{0}^{i}:=s^{i}{ }_{j} y^{j}$ and $G_{\alpha}^{i}$ denote the geodesic coefficients of $\alpha$.

Given any Randers space $(M, F=\alpha+\beta)$, the $S$-curvature takes the following form:

$$
\mathbf{S}=(n+1)\left\{\frac{e_{00}}{2 F}-s_{0}-\rho_{0}\right\}
$$

where $\rho=\ln \left(\sqrt{1-\|\beta\|_{\alpha}^{2}}\right)$ and $\rho_{0}=\rho_{x^{i}} y^{i}$. Akbar-Zadeh in [2] studied another nonRiemannian quantity $H$-curvature, which is invariant by special projective and $C$ projective algebras [15]. At every point $x \in M, \Xi_{y}=\Xi_{i}(y) d x^{i}$ and $H=H_{i j} d x^{i} \otimes d x^{j}$ are defined as follows:

$$
\begin{aligned}
\Xi_{i} & =y^{m} S_{. i \mid m}-S_{\mid i} \\
H_{i j} & =\frac{1}{2} S_{. i . j \mid m} y^{m}=\frac{1}{4}\left(\Xi_{i . j}+\Xi_{j . i}\right)
\end{aligned}
$$

where "." and "|" denote the vertical and horizontal covariant derivatives, respectively, with respect to the Berwald connection. The quantity $\Xi$ has been introduced by Zhongmin Shen using the $S$-curvature, cf. $[14,16]$. The above quantities do not depend on the choice of connection for performing horizontal derivatives and can be derived for the Finsler metric itself.

The notion of Riemann curvature for Riemann metrics is extended to Finsler metrics. For a vector $y \in T_{x} M_{0}$, the Riemann curvature $R_{y}: T_{x} M \rightarrow T_{x} M$ is defined by

$$
R_{y}(u):=R_{k}^{i}(y) u^{k} \frac{\partial}{\partial x^{i}},
$$

where

$$
R_{k}^{i}(y)=2 \frac{\partial G^{i}}{\partial x^{k}}-\frac{\partial^{2} G^{i}}{\partial x^{j} \partial y^{k}} y^{j}+2 G^{j} \frac{\partial^{2} G^{i}}{\partial y^{j} \partial y^{k}}-\frac{\partial G^{i}}{\partial y^{j}} \frac{\partial G^{j}}{\partial y^{k}} .
$$

The family $R:=\left\{R_{y}\right\}_{y \in T M_{0}}$ is called the Riemann curvature. We define the Ricci curvature as the trace of $R_{y}$, i.e., $\operatorname{Ric}(x, y):=\operatorname{trace}\left(R_{y}\right)=(n-1) R_{m}^{m}(y)$. 
Every vector field $X$ on a manifold $M$ induces naturally an infinitesimal coordinate transformations on $T M$ given by $\left(x^{i}, y^{i}\right) \rightarrow\left(\bar{x}^{i}, \bar{y}^{i}\right)$, given by

$$
\bar{x}^{i}=x^{i}+V^{i} d t, \quad \bar{y}^{i}=y^{i}+y^{k} \frac{\partial V^{i}}{\partial x^{k}} d t .
$$

Using this coordinates transformation, we may consider the notion of the complete lift $\hat{X}$ of $V$ to a vector field on $T M_{0}$ given by

$$
\hat{X}=X^{i} \frac{\partial}{\partial x^{i}}+y^{k} \frac{\partial X^{i}}{\partial x^{k}} \frac{\partial}{\partial y^{i}}
$$

It is a notable remark on the Lie derivative computations that, $\mathcal{L}_{\hat{X}} y^{i}=0$ and the differential operators $\mathcal{L}_{\hat{X}}, \quad \frac{\partial}{\partial x^{i}}$, exterior differential operator $d$ and $\frac{\partial}{\partial y^{i}}$ commute. The vector field $\hat{X}$ is called a projective vector field, if there is a function $\mathrm{P}$ (called the projective factor) on $T M_{0}$ such that $\mathcal{L}_{\hat{X}} G^{i}=P y^{i}$, cf. [1]. In this case, given any appropriate $t$, the local flow $\left\{\phi_{t}\right\}$ associated with $X$ is a projective transformation. A projective vector field is said to be affine if $P=0$. It is well-known that every Killing vector field is affine. Recall that, given any projective vector field $X$ on a Riemannian space, the projective factor $P=P(x, y)$ is linear with respect to $y$ and also it is the natural lift of a closed 1-form on $M$ to a function on $T M_{0}$, while in the Finslerian setting these issues are non-Riemannian features. Consider the following conventional definitions of a projective vector field $X ; X$ is said to be (cf. [10])

1. special if $\mathcal{L}_{\hat{X}} E=0$, or equivalently, $P(x, y)=P_{i}(x) y^{i}$.

2. $C$-projective if $P_{i \mid j}=P_{j \mid i}$.

3. $H$-invariant if $\mathcal{L}_{\hat{X}} H=0$, equivalently, $P_{j k \mid l}=P_{j l \mid k}$.

The projective factor $P$ for a projective vector field $X$ on a Riemannian manifold is simultaneously a special and a C-projective vector field. The following theorem provides the equivalence conditions of $C$-projective vector fields [13]:

Theorem 2.1. [13] Let $(M, F=\alpha+\beta)$ be a Randers space and $V$ is a projective vector field $V \in \chi(M)$. The following statements are equivalent:

1. $V$ is $C$-projective,

2. $\mathcal{L}_{V} \Xi=0$,

3. $\mathcal{L}_{V} \Sigma=0$.

where $\Sigma=\Sigma_{i j} d x^{i} \otimes d x^{j}$ is defined as follows:

$$
\Sigma_{i j}=\frac{1}{n+1}\left(S_{. i \mid j}-S_{. j \mid i}\right)
$$

Let $\hat{V}$ be a projective vector field. We have the following identities for arbitrary tensors $T_{i \mid j}$ and $T_{i j \mid k}[1]$ :

$$
\begin{aligned}
\mathcal{L}_{\hat{X}} T_{i \mid j} & =\left(\mathcal{L}_{\hat{X}} T_{i}\right)_{\mid j}-\left(\mathcal{L}_{\hat{X}} G_{i}^{r}\right) T_{r . j}-\left(\mathcal{L}_{\hat{X}} G_{i j}^{r}\right) T_{r} \\
\mathcal{L}_{\hat{X}} T_{i j \mid k} & =\left(\mathcal{L}_{\hat{X}} T_{i j}\right)_{\mid k}-\left(\mathcal{L}_{\hat{X}} G_{k}^{r}\right) T_{i j . r}-\left(\mathcal{L}_{\hat{X}} G_{i}^{r}\right) T_{r j}-\left(\mathcal{L}_{\hat{X}} G_{j}^{r}\right) T_{i r}
\end{aligned}
$$




\section{Projective vector field v.s. $\Xi$-curvature}

Let $F=\alpha+\beta$ be a Randers metric. Due to (2.4), $\Xi$-curvature is directly obtained as follows:

$$
\Xi_{i}=\frac{e_{i 0 \mid 0}}{F}-\frac{e_{00 \mid 0} u_{i}}{2 F^{2}}-\frac{e_{00 \mid i}}{2 F}-\Theta_{i}
$$

where $\Theta_{i}$ is $\Theta_{i}:=s_{i \mid 0}-s_{0 \mid i}$.

Using spray coefficients of Randers metric (2.3) and the general formula of horizontal derivatives (2.2) one can obtain the following:

$$
\begin{aligned}
e_{00 \mid 0}= & e_{00 ; 0}-\frac{4 e_{00}^{2}}{F}+8 e_{00} s_{0}-4 \alpha\left(q_{00}+\beta t_{0}+s_{0}^{2}\right) \\
e_{00 \mid i}= & e_{00 ; i}-\frac{5 e_{00} e_{i 0}}{F}+2 e_{i 0} s_{0}+\frac{2 e_{00}^{2} u_{i}}{F^{2}}+4 e_{00} s_{i} \\
& -2 \alpha\left(q_{i 0}+\beta t_{i}+s_{0} s_{i}\right)-2 \alpha_{i}\left(q_{00}+\beta t_{0}+s_{0}^{2}\right) \\
e_{i 0 \mid 0}= & e_{i 0 ; 0}-\frac{7 e_{i 0} e_{00}}{2 F}+3 e_{i 0} s_{0}+\frac{e_{00}^{2} u_{i}}{F^{2}}+2 e_{00} s_{i} \\
& -\alpha_{i}\left(q_{00}+\beta t_{0}+s_{0}^{2}\right)-\alpha\left(q_{i 0}+\beta t_{i}+s_{0} s_{i}\right) \\
e_{i j \mid 0}= & e_{i j ; 0}-2 e_{i j}\left(\frac{e_{00}}{2 F}-s_{0}\right)-e_{i 0}\left(\frac{e_{j 0}}{F}-\frac{e_{00} u_{j}}{2 F^{2}}-s_{j}\right) \\
& -e_{j 0}\left(\frac{e_{i 0}}{F}-\frac{e_{00} u_{i}}{2 F^{2}}-s_{i}\right) \\
& -\alpha_{i}\left(q_{j 0}+b_{j} t_{0}+s_{0} s_{j}\right)-\alpha_{j}\left(q_{i 0}+b_{i} t_{0}+s_{0} s_{i}\right) \\
& -\alpha\left(q_{i j}+q_{j i}+b_{i} t_{j}+b_{j} t_{i}+2 s_{i} s_{j}\right)
\end{aligned}
$$

where ";" is the covariant derivatives with respect to $\alpha$.

By substituting (3.2) in (3.1), we obtain:

$$
\begin{aligned}
\Xi_{i} & =\frac{1}{2 F^{3}}\left[\left(-e_{00 ; i}+2 e_{i 0 ; 0}-4 e_{i 0} s_{0}\right) F^{2}-u_{i}\left(-4\left(\beta t_{0}+s_{0}^{2}+q_{00}\right) \alpha+8 s_{0} e_{00}+e_{00 ; 0}\right) F\right. \\
& \left.+4 e_{00}^{2} u_{i}+2 e_{i 0} e_{00} F+4 F^{2} \alpha\left(b_{i} t_{0}+s_{i} s_{0}+q_{0 i}\right)-2 A_{i 0} F^{3}\right]
\end{aligned}
$$

where $u_{i}=\frac{y_{i}}{F}$.

Proof of Theorem 1.1 Let us suppose $X$ be a $C$-projective vector field. Then by Theorem 2.1, we have $\mathcal{L}_{\hat{X}} \Xi_{i}=0$. Suppose $\mathcal{L}_{\hat{X}} \alpha^{2}=t_{00}$. It is easy to see that every $C$-projective vector field is a projective vector field and every projective vector field of $F=\alpha+\beta$, is a projective vector field of $\alpha$ (see [12]), then there is a projective factor $\eta=\eta(x, y)$ which is linear with respect to $y$. By using the Lie identity of (2.7) we obtain:

$$
\begin{aligned}
\mathcal{L}_{\hat{X}} e_{i 0 ; 0} & =\left(\mathcal{L}_{\hat{X}} e_{i 0}\right)_{; 0}-2 e_{00} \eta_{i}-3 e_{i 0} \eta \\
\mathcal{L}_{\hat{X}} e_{00 ; i} & =\left(\mathcal{L}_{\hat{X}} e_{00}\right)_{; 0}-4 e_{00} \eta_{i}-2 e_{i 0} \eta \\
\mathcal{L}_{\hat{X}} e_{00 ; 0} & =\left(\mathcal{L}_{\hat{X}} e_{00}\right)_{; 0}-8 e_{00} \eta
\end{aligned}
$$


Then by substituting (3.3) and using the Maple program, we obtain the following:

$$
\begin{aligned}
\mathcal{L}_{\hat{X}} \Xi_{i} & =\frac{3 \Xi_{i} \mathcal{L}_{\hat{X}} F}{F}- \\
& -\frac{1}{2 F^{3}}\left(-8 u_{i} e_{00} \mathcal{L}_{\hat{X}} e_{00}-4 F^{2} s_{0} \mathcal{L}_{\hat{X}} e_{i 0}-4 F^{2} e_{i 0} \mathcal{L}_{\hat{X}} s_{0}+2 F e_{i 0} \mathcal{L}_{\hat{X}} e_{00}\right. \\
& +2 F e_{00} \mathcal{L}_{\hat{X}} e_{i 0}+6 F^{2} \mathcal{L}_{\hat{X}} F A_{i 0}+ \\
& +\left(-4 F \alpha \mathcal{L}_{\hat{X}} u_{i}-4 u_{i} \alpha \mathcal{L}_{\hat{X}} F-4 F u_{i} \frac{t_{00}}{2 \alpha}\right)\left(\beta t_{0}+s_{0}^{2}+q_{00}\right) \\
& +4 F^{2} \alpha\left(\mathcal{L}_{\hat{X}} b_{i} t_{0}+\mathcal{L}_{\hat{X}} b_{i} t_{0}+\mathcal{L}_{\hat{X}} s_{0} s_{i}+s_{0} \mathcal{L}_{\hat{X}} s_{i}+\mathcal{L}_{\hat{X}} q_{i 0}\right)+2 e_{i 0} e_{00} \mathcal{L}_{\hat{X}} F \\
& -8 F \mathcal{L}_{\hat{X}} F s_{0} e_{i 0}+s_{0} \mathcal{L}_{\hat{X}} F u_{i} e_{00}+8 F s_{0} e_{00} \mathcal{L}_{\hat{X}} u_{i}+ \\
& +8 F u_{i} e_{00} \mathcal{L}_{\hat{X}} s_{0}+8 F u_{i} s_{0} \mathcal{L}_{\hat{X}} e_{00} \\
& -4 F u_{i} \alpha\left(\mathcal{L}_{\hat{X}} \beta t_{0}+2 s_{0} \mathcal{L}_{\hat{X}} s_{0}+\mathcal{L}_{\hat{X}} t_{0} \beta+\mathcal{L}_{\hat{X}} q_{00}\right)+ \\
& +2 F^{3} \mathcal{L}_{\hat{X}} A_{i 0}+2 F \mathcal{L}_{\hat{X}} F e_{00 ; i} \\
& -4 F \mathcal{L}_{\hat{X}} F e_{i 0 ; 0}+F u_{i}\left(\left(\mathcal{L}_{\hat{X}} e_{00}\right)_{; 0}\right)-8 \eta e_{00}+e_{00 ; 0} F \mathcal{L}_{\hat{X}} u_{i}+e_{00 ; 0} u_{i} \mathcal{L}_{\hat{X}} F \\
& +\left(4\left(2 F \mathcal{L}_{\hat{X}} F \alpha+F^{2} \frac{t_{00}}{2 \alpha}\right)\right)\left(b_{i} t_{0}+s_{0} s_{i}+q_{i 0}\right)+ \\
& \left.+F^{2}\left(\mathcal{L}_{\hat{X}} e_{00}\right)_{; i}-2 \eta_{i} e_{00}-3 \eta e_{i 0}\right) \\
& \left.\left.-2 F^{2}\left(\mathcal{L}_{\hat{X}} e_{i 0}\right)_{; 0}-4 \eta_{i} e_{00}-2 \eta e_{i 0}\right)-4 e_{00}^{2} \mathcal{L}_{\hat{X}} u_{i}\right) \\
& =-2 F^{4}\left(R a t_{i}+\alpha \operatorname{Irat} t_{i}\right)
\end{aligned}
$$

where Rat $_{i}=A_{0}+A_{2} \alpha^{2}+A_{4} \alpha^{4}+A_{6} \alpha^{6}$ and Irrat $_{i}=A_{7} \alpha^{6}+A_{5} \alpha^{4}+A_{3} \alpha^{2}+A_{1}$ and the terms $A_{0}, \cdots, A_{6}$ are respectively given in Appendix 1.

$$
\begin{aligned}
& A_{0}=8 e_{00} t_{00} \beta^{2} s_{0} y_{i}-4 e_{00}^{2} t_{00} y_{i} \beta+e_{00 ; 0} t_{00} \beta^{2} y_{i} \\
& A_{1}=-2 t_{00}\left(8 e_{00} \beta s_{0} y_{i}-4 e_{00}^{2} y_{i}+e_{00 ; 0} \beta y_{i}\right)
\end{aligned}
$$

The equation (3.4) is equivalent to Rat $_{i}=0$ and Irrat $_{i}=0,(i=1, \ldots, n)$. The system of equations Rat $_{i}=0$ and Irrat $_{i}=0$ is itself equivalent to the system of equations Rat $_{i}-\beta$ Irrat $_{i}=0$ and Irrat $_{i}=0$. By using Maple, we obtain the followings:

$$
\begin{aligned}
& \text { Rat }_{i}-\beta \text { Irrat }_{i}= \\
& =\left(\alpha^{2}-\beta^{2}\right)\left\{\left[-32 \mathcal{L}_{\hat{X}} \beta b_{i} t_{0}-40 \mathcal{L}_{\hat{X}} \beta s_{0} s_{i}-24 \mathcal{L}_{\hat{X}} b_{i} \beta t_{0}+8 \mathcal{L}_{\hat{X}} b_{i} s_{0}^{2}\right.\right. \\
& -16 \mathcal{L}_{\hat{X}} s_{0} \beta s_{i}+16 \mathcal{L}_{\hat{X}} s_{0} b_{i} s_{0}-16 \mathcal{L}_{\hat{X}} s_{i} \beta s_{0}+8 \mathcal{L}_{\hat{X}} t_{0} \beta b_{i}-24 a_{i 0} \mathcal{L}_{\hat{X}} \beta \\
& +8 e_{i 0} \mathcal{L}_{\hat{X}} s_{0}-8 e_{i 0} \eta-12 \mathcal{L}_{\hat{X}} a_{i 0} \beta+8 \mathcal{L}_{\hat{X}} e_{i 0} s_{0}-24 \mathcal{L}_{\hat{X}} \beta q_{0 i}-16 \mathcal{L}_{\hat{X}} \beta q_{i 0} \\
& \left.+8 \mathcal{L}_{\hat{X}} b_{i} q_{00}+8 \mathcal{L}_{\hat{X}} q_{00} b_{i}-16 \mathcal{L}_{\hat{X}} q_{i 0} \beta-2 \mathcal{L}_{\hat{X}} e_{00 ; i}+4 \mathcal{L}_{\hat{X}} e_{i 0 ; 0}\right] \alpha^{4} \\
& +\left[-24 a_{i 0}\left(\mathcal{L}_{\hat{X}} \beta\right) \beta^{2}-64 e_{00} \mathcal{L}_{\hat{X}} \beta b_{i} s_{0}-16 e_{00} \mathcal{L}_{\hat{X}} b_{i} \beta s_{0}-16 e_{00} \mathcal{L}_{\hat{X}} s_{0} \beta b_{i}\right. \\
& +16 e_{00} \beta b_{i} \eta+40 e_{i 0}\left(\mathcal{L}_{\hat{X}} \beta\right) \beta s_{0}+8 e_{i 0} \mathcal{L}_{\hat{X}} s_{0} \beta^{2}-8 e_{i 0} \beta^{2} \eta-4 \mathcal{L}_{\hat{X}} a_{i 0} \beta^{3}
\end{aligned}
$$




$$
\begin{aligned}
& -16 \mathcal{L}_{\hat{X}} e_{00} \beta b_{i} s_{0}+8 \mathcal{L}_{\hat{X}} e_{i 0} \beta^{2} s_{0}+40\left(\mathcal{L}_{\hat{X}} \beta\right) \beta t_{0} y_{i}+32 \mathcal{L}_{\hat{X}} \beta s_{0}^{2} y_{i} \\
& +16 \mathcal{L}_{\hat{X}} s_{0} \beta s_{0} y_{i}+8 \mathcal{L}_{\hat{X}} t_{0} \beta^{2} y_{i}+8 \mathcal{L}_{\hat{X}} y_{i} \beta^{2} t_{0}+8 \mathcal{L}_{\hat{X}} y_{i} \beta s_{0}^{2}-8 t_{00} \beta b_{i} t_{0} \\
- & 28 t_{00} \beta s_{0} s_{i}+20 t_{00} b_{i} s_{0}^{2}-24 a_{i 0} t_{00} \beta+8 e_{00}^{2} \mathcal{L}_{\hat{X}} b_{i}-16 e_{00} e_{i 0} \mathcal{L}_{\hat{X}} \beta \\
& +16 e_{00} \mathcal{L}_{\hat{X}} e_{00} b_{i}-4 e_{00} \mathcal{L}_{\hat{X}} e_{i 0} \beta-16 e_{00} \mathcal{L}_{\hat{X}} s_{0} y_{i}-16 e_{00} \mathcal{L}_{\hat{X}} y_{i} s_{0} \\
& +16 e_{00} \eta y_{i}-8 e_{00 ; 0} \mathcal{L}_{\hat{X}} \beta b_{i}-2 e_{00 ; 0} \mathcal{L}_{\hat{X}} b_{i} \beta-10 e_{00 ; i}\left(\mathcal{L}_{\hat{X}} \beta\right) \beta-4 e_{i 0} \mathcal{L}_{\hat{X}} e_{00} \beta \\
& +20 e_{i 0} t_{00} s_{0}+20 e_{i 0 ; 0}\left(\mathcal{L}_{\hat{X}} \beta\right) \beta-16 \mathcal{L}_{\hat{X}} e_{00} s_{0} y_{i}-2 \mathcal{L}_{\hat{X}} e_{00 ; 0} \beta b_{i}-2 \mathcal{L}_{\hat{X}} e_{00 ; i} \beta^{2} \\
& +4 \mathcal{L}_{\hat{X}} e_{i 0 ; 0} \beta^{2}+32 \mathcal{L}_{\hat{X}} \beta q_{00} y_{i}+8 \mathcal{L}_{\hat{X}} q_{00} \beta y_{i}+8 \mathcal{L}_{\hat{X}} y_{i} \beta q_{00}-12 t_{00} \beta q_{0 i} \\
& \left.-16 t_{00} \beta q_{i 0}+20 t_{00} b_{i} q_{00}-2 e_{00 ; 0} \mathcal{L}_{\hat{X}} y_{i}-5 e_{00 ; i} t_{00}+10 e_{i 0 ; 0} t_{00}-2 \mathcal{L}_{\hat{X}} e_{00 ; 0} y_{i}\right] \alpha^{2} \\
- & \left.24 e_{00}^{2} \mathcal{L}_{\hat{X}}(\beta) \beta b_{i}+24 e_{00}^{2} \mathcal{L}_{\hat{X}} \beta y_{i}+12 e_{00}^{2} t_{00} b_{i}-24 e_{00} t_{00} s_{0} y_{i}-3 e_{00 ; 0} t_{00} y_{i}\right\} \\
+ & 12 e_{00}^{2}\left\{\left[\left(-2 \beta b_{i}+2 y_{i}\right) \mathcal{L}_{\hat{X}} \beta+b_{i} t_{00}\right] \alpha^{2}-y_{i} t_{00} \beta\right\}
\end{aligned}
$$

By the above equation, for any point $x \in M$, the irreducible polynomial $\alpha^{2}-\beta^{2}$ divides $e_{00}$ or $\left[\left(-2 \beta b_{i}+2 y_{i}\right) \mathcal{L}_{\hat{X}} \beta+b_{i} t_{00}\right] \alpha^{2}-y_{i} t_{00} \beta$. In the first case, for a function $c \in C^{\infty}(M), e_{00}=2 c(x)\left(\alpha^{2}-\beta^{2}\right)$ which means that $F$ is of isotropic $S$-curvature. In the second case, we have

$$
\left[\left(-2 \beta b_{i}+2 y_{i}\right) \mathcal{L}_{\hat{X}} \beta+b_{i} t_{00}\right] \alpha^{2}-y_{i} t_{00} \beta=\eta_{i}(x, y)\left(\alpha^{2}-\beta^{2}\right)
$$

where $\eta_{i}$ are polynomials of degree two. By contracting above equation with $b^{i}$, we obtain

$$
b^{2}\left(\mathcal{L}_{\hat{X}}\left(\alpha^{2}-\beta^{2}\right)-\left(\beta^{2} \mathcal{L}_{\hat{X}} \alpha^{2}-\alpha^{2} \mathcal{L}_{\hat{X}} \beta^{2}\right)=\eta(x, y)\left(\alpha^{2}-\beta^{2}\right)\right.
$$

where $\eta=\eta_{i}(x, y) b^{i}$ is a polynomial of degree two on $T M$.

By Irrat $_{i}=0$, we have $\mathcal{L}_{\hat{X}} \alpha^{2}=\sigma(x) \alpha^{2}$ which means that $\hat{X}$ is a conformal vector field of $\alpha$, or $\alpha^{2}$ divides $\left(8 e_{00} \beta s_{0} y_{i}-4 e_{00}^{2} y_{i}+e_{00 ; 0} \beta y_{i}\right)$. By contracting it with $b^{i}$, we obtain

$$
\left(8 e_{00} \beta s_{0}-4 e_{00}^{2}+e_{00 ; 0} \beta\right)=\lambda(x, y) \alpha^{2}
$$

where $\lambda(x, y)$ is a polynomial of degree two.

Example 3.1. Let $M=\mathbb{R}^{2}$ and $F(x, y, u, v)=\sqrt{u^{2}+v^{2}}+a u+b v$, where $a, b \in \mathbb{R}$ and $a^{2}+b^{2}<1$. Since $F$ is Berwaldian, $S=0$ and $\Xi=0$, i.e., every projective vector field is a $C$-projective vector field. Let $V=x \frac{\partial}{\partial x}+y \frac{\partial}{\partial y}$. Then by a dircet calculation, we have $\mathcal{L}_{\hat{X}} \alpha^{2}=2 \alpha^{2}$ and $\mathcal{L}_{\hat{X}} \beta=\beta$. If we substitute them in (3.4) we obtain:

$$
\left[\left(-2 b_{i} \beta+2 y_{i}\right) \beta+2 b_{i} \alpha^{2}\right] \alpha^{2}-2 y_{i} \alpha^{2} \beta=\eta_{i}(x, y)\left(\alpha^{2}-\beta^{2}\right)
$$

By contracting (4.4) with $y^{i}$ we have $\eta(x, y)=2 \beta \alpha^{2}$. In this case all of the conditions Theorem 1.1 is held.

Here we make an example which satiesfies condition 1 and 3 of Theorem 1.1.

Example 3.2. Let $M=\mathbb{B}^{2}(1)$ open ball on $\mathbb{R}^{2}$ and let $F$ be Funk metric as:

$$
\theta=\frac{\sqrt{|y|^{2}-|x|^{2}|y|^{2}+<x, y>^{2}}}{1-|x|^{2}}+\frac{<x, y>}{1-|x|^{2}}
$$


By [6] one can see that Funk metric is of constant $S$-curvature $S=\frac{1}{2} \theta$. It is easy to see that the vector field $\left.V=\left(x^{i}<a, x\right\rangle-a^{i}\right) \frac{\partial}{\partial x^{i}}$ is a Killing vector field of $\alpha:=\frac{\sqrt{\left.|y|^{2}-|x|^{2}|y|^{2}+<x, y\right\rangle^{2}}}{1-|x|^{2}}$. By direct calculation we have

$$
\mathcal{L}_{\hat{V}}(\beta)=-<a, y>
$$

where $\beta:=\frac{<x, y\rangle}{1-|x|^{2}}$. So conditions 1 and 3 of Theorem 1.1 hold. Here, we examine condition 2 and 4 . Since $s_{j}=0$ and $F$ is of constant $S$-curvature, we have $r_{00}=$ $\alpha^{2}-\beta^{2}$. By taking the covariant derivative with respect to $\alpha$, we have

$$
r_{00 ; 0}=-2 \beta\left(\alpha^{2}-\beta^{2}\right) .
$$

If we substitute (3.8) in condition 4, we have

$$
\frac{|y|^{2}}{1-|x|^{2}}\left\{2|y|^{2}\left(1-|x|^{2}\right)+<x, y>^{2}\right\}=\left(|y|^{2}\left(1-|x|^{2}\right)+<x, y>^{2}\right) \lambda(x, y)
$$

but $\lambda(x, y)$ should be a polynomial of degree 2, which is a contradiction. Now we consider condition 2. Since $\mathcal{L}_{\hat{V}} \alpha^{2}=0$, condition 2 reduces to

$$
\alpha^{2}\left(1-b^{2}\right) \mathcal{L}_{\hat{V}} \beta^{2}=\eta(x, y)\left(\alpha^{2}-\beta^{2}\right)
$$

Then by substituting $b^{2}=|x|^{2}$ and $\mathcal{L}_{\hat{V}} \beta^{2}$ we have

$$
-2<a, y><x, y>\left\{|y|^{2}+\frac{<x, y>^{2}}{1-|y|^{2}}\right\}=|y|^{2} \eta_{V}(x, y)
$$

where $\eta_{V}$ is a polynomial of degree two. If we write the extended form of (3.9) we have

$$
\begin{aligned}
\left(a_{1} y_{1}+a_{2} y_{2}\right) \quad & \left(x_{1} y_{1}+x_{2} y_{2}\right)\left\{y_{1}^{2}+y_{2}^{2}+\frac{x_{1}^{2} y_{1}^{2}+x_{2}^{2} y_{2}^{2}+2 x_{1} x_{2} y_{1} y_{2}}{1-|x|^{2}}\right\} \\
& =\left(y_{1}^{2}+y_{2}^{2}\right)\left(a_{11}(x) y_{1}^{2}+2 a_{12}(x) y_{1} y_{2}+a_{22} y_{2}^{2}\right)
\end{aligned}
$$

By comparing left and right side of above equation we have $a_{1} x_{1} x_{2}^{2}+a_{2} x_{1}^{2} x_{2}=0$, i.e. $a=0$ which is contradition. Therefore, there is not any $\eta(x, y)$ to applies condition 2.

Here we make an example which satisfies condition 2 and 3 of Theorem 1.1.

Example 3.3. Let $\alpha$ be the Bergman metric on $D=\left\{x \in \mathbb{R}^{2 N}:|x|<1\right\}$ and $f=\frac{1}{2} \ln \left(1-|x|^{2}\right)$ be the potential of $\alpha$, and $J$ be the complex structure. B. Chen and L. Zhao [5] proved Randers metric

$$
F_{\epsilon}(x, y)=\sqrt{\alpha(y, y)}+d f(\epsilon y-J y), \quad \epsilon \neq 0
$$


is of scalar flag curvature and neither projectively flat nor of isotropic S-curvature. That is the condition 1 of Theorem 1.1 is not held. Let $\hat{V}$ be a Killing vector field of $F$, i. e. condition 2 and 3 are established automatically. By [5] we have

$$
\begin{gathered}
b_{k}=-J_{k}^{i} f_{i}, \quad s_{k}=-f_{k}, \quad a_{k j}=-\frac{1}{2}\left(f_{s i} J_{k}^{s} J_{j}^{i}+f_{j k}\right), \quad r_{00}+2 s_{0} \beta=0 \\
e_{00}(\epsilon)=2 \epsilon \beta\left(\beta+\epsilon f_{0}\right), \quad e_{00 ; 0}(\epsilon)=4 \epsilon \beta f_{0}\left(\beta+\epsilon f_{0}+f_{0}\right)
\end{gathered}
$$

By substituting (3.10) in condition 4 Theorem 1.1, we find out that the term

$$
4 \epsilon \beta^{2}\left(-3 f_{0}\left(\beta+\epsilon f_{0}\right)+f_{0}^{2}-4 \epsilon\left(\beta+\epsilon f_{0}\right)^{2}\right)
$$

should be a multiple of $\alpha^{2}$, which is impossible. Thus condition 4 is not true.

\section{Projective vector fields vs. $H$-projective}

Let $F=\alpha+\beta$ be a Randers metric. Due to (2.6), $H$-curvature can be directly obtained as follows:

$$
4 H_{i j}=\frac{2 e_{i j \mid 0}}{F}-\frac{2 e_{i 0 \mid 0} u_{j}}{F^{2}}-\frac{2 e_{j 0 \mid 0} u_{i}}{F^{2}}-\frac{e_{00 \mid 0} F_{i j}}{F^{2}}+\frac{2 e_{00 \mid 0} u_{i} u_{j}}{F^{3}}
$$

By substituting (3.2) in (4.1) we obtain:

Proof of Theorem 1.2 Let us suppose $X$ be a $H$-invariant vector field, i. e. $\mathcal{L}_{\hat{X}} H_{i j}=0$ Suppose $\mathcal{L}_{\hat{X}} \alpha^{2}=t_{00}$, then by using Maple program and using equations (4.1), (3.2) and (3.3) we obtain the following:

$$
H_{i j}=-2 F^{4}\left(\text { Rat }_{i j}+\alpha \operatorname{Irrat}_{i j}\right)
$$

where Rat $_{i j}=A_{0}+A_{2} \alpha^{2}+\cdots+A_{10} \alpha^{10}$ and $\operatorname{Irrat}_{i j}=A_{9} \alpha^{8}+\cdots+A_{1}$ and the terms $A_{0}, \cdots, A_{10}$ are respectively given in Appendix 2 .

$$
A_{1}=3 t_{00} \beta y_{i} y_{j}\left(-40 e_{00} \beta s_{0}+16 e_{00}^{2}-5 e_{00 ; 0} \beta\right)
$$

The equation (4.2) is equivalent to $\operatorname{Rat}_{i j}=0$ and $\operatorname{Irrat}_{i j}=0$. The system of equations $R_{a t} t_{i j}=0$ and Irrat $_{i j}=0$ is itself equivalent to the system of equations Rat $_{i j}-\beta$ Irrat $_{i j}=0$ and Irrat $_{i j}=0$. By using Maple we obtain the followings:

$$
\begin{aligned}
& \text { Rat }_{i j}-\beta \text { Irrat }_{i j}= \\
& =\left(\alpha^{2}-\beta^{2}\right)\left\{\left[-2\left(\mathcal{L}_{\hat{X}} b_{i} t_{j}+\mathcal{L}_{\hat{X}} b_{j} t_{i}+2 \mathcal{L}_{\hat{X}} s_{i} s_{j}+2 \mathcal{L}_{\hat{X}} s_{j} s_{i}+\mathcal{L}_{\hat{X}} t_{i} b_{j}\right.\right.\right. \\
& \left.\left.+\mathcal{L}_{\hat{X}} t_{j} b_{i}+\mathcal{L}_{\hat{X}} q_{i j}+\mathcal{L}_{\hat{X}} q_{j i}\right)\right] \alpha^{8}+\left[12 \mathcal{L}_{\hat{X}} \beta \beta b_{i} t_{j}+12 \mathcal{L}_{\hat{X}} \beta \beta b_{j} t_{i}+40 \mathcal{L}_{\hat{X}} \beta \beta s_{i} s_{j}\right. \\
& -72 \mathcal{L}_{\hat{X}} \beta b_{i} b_{j} t_{0}-44 \mathcal{L}_{\hat{X}} \beta b_{i} s_{0} s_{j}-44 \mathcal{L}_{\hat{X}} \beta b_{j} s_{0} s_{i}-10 e_{i 0} \mathcal{L}_{\hat{X}} \beta s_{j}-20 e_{i j} \mathcal{L}_{\hat{X}} \beta s_{0} \\
& -10 e_{j 0} \mathcal{L}_{\hat{X}} \beta s_{i}+20 \mathcal{L}_{\hat{X}} \beta \beta q_{i j}+20 \mathcal{L}_{\hat{X}} \beta \beta q_{j i}-24 \mathcal{L}_{\hat{X}} \beta b_{i} q_{0 j}-12 \mathcal{L}_{\hat{X}} \beta b_{i} q_{j 0} \\
& -28 \mathcal{L}_{\hat{X}} \beta b_{j} q_{0 i}-12 \mathcal{L}_{\hat{X}} \beta b_{j} q_{i 0}+5 t_{00} b_{i} t_{j}+5 t_{00} b_{j} t_{i}+10 t_{00} s_{i} s_{j}-12 \mathcal{L}_{\hat{X}} s_{i} \beta^{2} s_{j} \\
& -12 \mathcal{L}_{\hat{X}} s_{j} \beta^{2} s_{i}-6 \mathcal{L}_{\hat{X}} t_{i} \beta^{2} b_{j}-2 \mathcal{L}_{\hat{X}} t_{j} \beta^{2} b_{i}+4 \mathcal{L}_{\hat{X}} t_{j} \beta^{2} b_{j}
\end{aligned}
$$




$$
\begin{aligned}
& +4 a_{i j} \mathcal{L}_{\hat{X}} \beta t_{0}+8 a_{i j} \mathcal{L}_{\hat{X}} s_{0} s_{0} \\
& +4 a_{i j} \mathcal{L}_{\hat{X}} t_{0} \beta-4 e_{00} \mathcal{L}_{\hat{X}} b_{i} s_{j}-4 e_{00} \mathcal{L}_{\hat{X}} b_{j} s_{i} \\
& -4 e_{00} \mathcal{L}_{\hat{X}} s_{i} b_{j}-4 e_{00} \mathcal{L}_{\hat{X}} s_{j} b_{i}+4 e_{00} b_{i} \eta_{j} \\
& +4 e_{00} b_{j} \eta_{i}-6 e_{i 0} \mathcal{L}_{\hat{X}} b_{j} s_{0}-6 e_{i 0} \mathcal{L}_{\hat{X}} s_{0} b_{j}+6 e_{i 0} \mathcal{L}_{\hat{X}} s_{j} \beta+2 \mathcal{L}_{\hat{X}} t_{j} \beta y_{i}+2 \mathcal{L}_{\hat{X}} t_{j} \beta y_{j} \\
& +2 \mathcal{L}_{\hat{X}} y_{i} \beta t_{j}-2 \mathcal{L}_{\hat{X}} y_{i} b_{j} t_{0}+4 \mathcal{L}_{\hat{X}} y_{i} s_{0} s_{j}+4 \mathcal{L}_{\hat{X}} y_{i} s_{j} t_{0} \\
& -6 e_{i 0} \beta \eta_{j}+6 e_{i 0} b_{j} \eta+12 e_{i j} \mathcal{L}_{\hat{X}} s_{0} \beta \\
& -12 e_{i j} \beta \eta-6 e_{j 0} \mathcal{L}_{\hat{X}} b_{i} s_{0}-6 e_{j 0} \mathcal{L}_{\hat{X}} s_{0} b_{i}+6 e_{j 0} \mathcal{L}_{\hat{X}} s_{i} \beta \\
& -6 e_{j 0} \beta \eta_{i}+6 e_{j 0} b_{i} \eta+4 \mathcal{L}_{\hat{X}} a_{i j} \beta t_{0} \\
& -4 \mathcal{L}_{\hat{X}} e_{00} b_{i} s_{j}-4 \mathcal{L}_{\hat{X}} e_{00} b_{j} s_{i}+6 \mathcal{L}_{\hat{X}} e_{i 0} \beta s_{j}+4 \mathcal{L}_{\hat{X}} a_{i j} \beta t_{0}+12 e_{i j} \mathcal{L}_{\hat{X}} s_{0} \beta \\
& -6 \mathcal{L}_{\hat{X}} e_{i 0} b_{j} s_{0}+12 \mathcal{L}_{\hat{X}} e_{i j} \beta s_{0}+6 \mathcal{L}_{\hat{X}} e_{j 0} \beta s_{i} \\
& -6 \mathcal{L}_{\hat{X}} e_{j 0} b_{i} s_{0}+2 \mathcal{L}_{\hat{X}} \beta t_{i} y_{j}-2 \mathcal{L}_{\hat{X}} b_{i} \beta^{2} t_{j} \\
& -8 \mathcal{L}_{\hat{X}} b_{i} b_{j} s_{0}^{2}-2 \mathcal{L}_{\hat{X}} b_{j} \beta^{2} t_{i}-8 \mathcal{L}_{\hat{X}} b_{j} b_{i} s_{0}^{2}+2 \mathcal{L}_{\hat{X}} \beta t_{j} y_{i}+8 \mathcal{L}_{\hat{X}} b_{i} \beta q_{0 j}+4 \mathcal{L}_{\hat{X}} b_{i} \beta q_{j 0} \\
& -8 \mathcal{L}_{\hat{X}} b_{i} b_{j} q_{00}-2 \mathcal{L}_{\hat{X}} b_{i} t_{0} y_{j}+8 \mathcal{L}_{\hat{X}} b_{j} \beta q_{0 i} \\
& +4 \mathcal{L}_{\hat{X}} b_{j} \beta q_{i 0}-8 \mathcal{L}_{\hat{X}} b_{j} b_{i} q_{00}-2 \mathcal{L}_{\hat{X}} b_{j} t_{0} y_{i} \\
& -8 \mathcal{L}_{\hat{X}} q_{00} b_{i} b_{j}+8 \mathcal{L}_{\hat{X}} q_{0 i} \beta b_{j}+8 \mathcal{L}_{\hat{X}} q_{0 j} \beta b_{i}+4 \mathcal{L}_{\hat{X}} q_{i 0} \beta b_{j}+4 \mathcal{L}_{\hat{X}} q_{j 0} \beta b_{i}+4 \mathcal{L}_{\hat{X}} s_{i} s_{0} y_{j} \\
& +4 \mathcal{L}_{\hat{X}} s_{i} t_{0} y_{j}+4 \mathcal{L}_{\hat{X}} s_{j} s_{0} y_{i}+4 \mathcal{L}_{\hat{X}} s_{j} t_{0} y_{i}-2 \mathcal{L}_{\hat{X}} t_{0} b_{i} y_{j}-2 \mathcal{L}_{\hat{X}} t_{0} b_{j} y_{i}+4 \mathcal{L}_{\hat{X}} t_{0} s_{i} y_{j} \\
& +4 \mathcal{L}_{\hat{X}} t_{0} s_{j} y_{i}+2 \mathcal{L}_{\hat{X}} y_{j} \beta t_{i}-2 \mathcal{L}_{\hat{X}} y_{j} b_{i} t_{0}+4 \mathcal{L}_{\hat{X}} y_{j} s_{0} s_{i}+4 \mathcal{L}_{\hat{X}} y_{j} s_{i} t_{0}-2\left(\mathcal{L}_{\hat{X}} e_{j 0}\right)_{; 0} b_{i} \\
& +4 \mathcal{L}_{\hat{X}} q_{0 i} y_{j}+4 \mathcal{L}_{\hat{X}} q_{0 j} y_{i}+4 \mathcal{L}_{\hat{X}} y_{i} q_{0 j}+4 \mathcal{L}_{\hat{X}} y_{j} q_{0 i} \\
& +4 \mathcal{L}_{\hat{X}} a_{i j} s_{0}^{2}-6 \mathcal{L}_{\hat{X}} q_{i j} \beta^{2}-10 e_{i j ; 0} \mathcal{L}_{\hat{X}} \beta \\
& +5 t_{00} q_{i j}+5 t_{00} q_{j i}-6 \mathcal{L}_{\hat{X}} q_{j i} \beta^{2}+4 a_{i j} \mathcal{L}_{\hat{X}} q_{00} \\
& -2 e_{00} \mathcal{L}_{\hat{X}} e_{i j}-2 e_{i 0 ; 0} \mathcal{L}_{\hat{X}} b_{j}-2 e_{i j} \mathcal{L}_{\hat{X}} e_{00} \\
& -2 e_{j 0 ; 0} \mathcal{L}_{\hat{X}} b_{i}+4 \mathcal{L}_{\hat{X}} a_{i j} q_{00}-2 \mathcal{L}_{\hat{X}} e_{i 0 ; 0} b_{j} \\
& +6 \mathcal{L}_{\hat{X}} e_{i j ; 0} \beta+4 \mathcal{L}_{\hat{X}} s_{0} \beta b_{j} s_{i}-16 \mathcal{L}_{\hat{X}} s_{0} b_{i} b_{j} s_{0} \\
& +12 \mathcal{L}_{\hat{X}} s_{i} \beta b_{j} s_{0}+8 \mathcal{L}_{\hat{X}} s_{i} \beta b_{j} t_{0}+12 \mathcal{L}_{\hat{X}} s_{j} \beta b_{i} s_{0} \\
& +8 \mathcal{L}_{\hat{X}} \beta b_{i} s_{j} t_{0}+8 \mathcal{L}_{\hat{X}} \beta b_{j} s_{i} t_{0}-8 \mathcal{L}_{\hat{X}} b_{i} \beta b_{j} t_{0} \\
& +12 \mathcal{L}_{\hat{X}} b_{i} \beta s_{0} s_{j}+8 \mathcal{L}_{\hat{X}} b_{i} \beta s_{j} t_{0}-8 \mathcal{L}_{\hat{X}} b_{j} \beta b_{i} t_{0} \\
& +12 \mathcal{L}_{\hat{X}} b_{j} \beta s_{0} s_{i}+8 \mathcal{L}_{\hat{X}} b_{j} \beta s_{i} t_{0}+4 \mathcal{L}_{\hat{X}} s_{0} \beta b_{i} s_{j} \\
& +8 \mathcal{L}_{\hat{X}} s_{j} \beta b_{i} t_{0}-8 \mathcal{L}_{\hat{X}} t_{0} \beta b_{i} b_{j}+8 \mathcal{L}_{\hat{X}} t_{0} \beta b_{i} s_{j} \\
& \left.+8 \mathcal{L}_{\hat{X}} t_{0} \beta b_{j} s_{i}-4 \mathcal{L}_{\hat{X}} e_{i 0} e_{j 0}-4 \mathcal{L}_{\hat{X}} e_{j 0} e_{i 0}\right] \alpha^{6} \\
& +\left[12 e_{i 0 ; 0} \mathcal{L}_{\hat{X}} \beta \beta b_{j}-26 e_{i j} t_{00} \beta s_{0}+36 e_{j 0} \mathcal{L}_{\hat{X}} \beta s_{0} y_{i}\right. \\
& -13 e_{j 0} t_{00} \beta s_{i}+21 e_{j 0} t_{00} b_{i} s_{0}-20 a_{i j} \mathcal{L}_{\hat{X}} \beta \beta^{2} t_{0} \\
& -24 a_{i j} \mathcal{L}_{\hat{X}} \beta \beta s_{0}^{2}-10 e_{i 0} \mathcal{L}_{\hat{X}} \beta \beta^{2} s_{j}-20 e_{i j} \mathcal{L}_{\hat{X}} \beta \beta^{2} s_{0} \\
& -10 e_{j 0} \mathcal{L}_{\hat{X}} \beta \beta^{2} s_{i}-10 \mathcal{L}_{\hat{X}} \beta \beta^{2} t_{i} y_{j} \\
& -10 \mathcal{L}_{\hat{X}} \beta \beta^{2} t_{j} y_{i}+44 \mathcal{L}_{\hat{X}} \beta b_{i} s_{0}^{2} y_{j}+44 \mathcal{L}_{\hat{X}} \beta b_{j} s_{0}^{2} y_{i} \\
& +12 e_{j 0 ; 0} \mathcal{L}_{\hat{X}} \beta \beta b_{i}-28 \mathcal{L}_{\hat{X}} \beta \beta q_{0 i} y_{j}-24 \mathcal{L}_{\hat{X}} \beta \beta q_{0 j} y_{i} \\
& +8 \mathcal{L}_{\hat{X}} \beta \beta q_{i 0} y_{j}+8 \mathcal{L}_{\hat{X}} \beta \beta q_{j 0} y_{i}+44 \mathcal{L}_{\hat{X}} \beta b_{i} q_{00} y_{j}
\end{aligned}
$$




$$
\begin{aligned}
& +44 \mathcal{L}_{\hat{X}} \beta b_{j} q_{00} y_{i}-12 t_{00} \beta b_{i} q_{0 j}-6 t_{00} \beta b_{i} q_{j 0} \\
& -18 t_{00} \beta b_{j} q_{0 i}-6 t_{00} \beta b_{j} q_{i 0}-7 t_{00} \beta t_{i} y_{j}-7 t_{00} \beta t_{j} y_{i} \\
& +28 t_{00} b_{i} b_{j} q_{00}-13 t_{00} b_{i} t_{0} y_{j}-13 t_{00} b_{j} t_{0} y_{i} \\
& -22 t_{00} s_{0} s_{i} y_{j}-22 t_{00} s_{0} s_{j} y_{i}-t_{00} \beta^{2} b_{i} t_{j}-t_{00} \beta^{2} b_{j} t_{i} \\
& +10 t_{00} \beta^{2} s_{i} s_{j}+28 t_{00} b_{i} b_{j} s_{0}^{2}+48 a_{i j} e_{00} \mathcal{L}_{\hat{X}} \beta s_{0} \\
& -24 a_{i j} \mathcal{L}_{\hat{X}} \beta \beta q_{00}-14 a_{i j} t_{00} \beta t_{0}-56 e_{00} e_{i 0} \mathcal{L}_{\hat{X}} \beta b_{j} \\
& +12 e_{00} e_{i j} \mathcal{L}_{\hat{X}} \beta \beta-56 e_{00} e_{j 0} \mathcal{L}_{\hat{X}} \beta b_{i}+24 e_{00} \mathcal{L}_{\hat{X}} \beta s_{i} y_{j} \\
& +24 e_{00} \mathcal{L}_{\hat{X}} \beta s_{j} y_{i}+14 e_{00} t_{00} b_{i} s_{j}+14 e_{00} t_{00} b_{j} s_{i} \\
& -14 e_{00 ; 0} \mathcal{L}_{\hat{X}} \beta b_{i} b_{j}+24 e_{i 0} e_{j 0} \mathcal{L}_{\hat{X}} \beta \beta+36 e_{i 0} \mathcal{L}_{\hat{X}} \beta s_{0} y_{j} \\
& -13 e_{i 0} t_{00} \beta s_{j}+21 e_{i 0} t_{00} b_{j} s_{0}+28 \mathcal{L}_{\hat{X}} \beta \beta b_{i} t_{0} y_{j} \\
& +28 \mathcal{L}_{\hat{X}} \beta \beta b_{j} t_{0} y_{i}+12 e_{j 0 ; 0} \mathcal{L}_{\hat{X}} \beta y_{i}+7 e_{j 0 ; 0} t_{00} b_{i} \\
& -18 t_{00} q_{0 i} y_{j}-14 t_{00} q_{0 j} y_{i}-14 a_{i j} t_{00} s_{0}^{2}+4 a_{i j} \mathcal{L}_{\hat{X}} t_{0} \beta^{3} \\
& -10 e_{i j ; 0} \mathcal{L}_{\hat{X}} \beta \beta^{2}+5 t_{00} \beta^{2} q_{i j}+5 t_{00} \beta^{2} q_{j i} \\
& +4 a_{i j} e_{00 ; 0} \mathcal{L}_{\hat{X}} \beta-14 a_{i j} t_{00} q_{00}+7 e_{00} e_{i j} t_{00} \\
& +14 e_{i 0} e_{j 0} t_{00}+12 e_{i 0 ; 0} \mathcal{L}_{\hat{X}} \beta y_{j}+7 e_{i 0 ; 0} t_{00} b_{j}-13 e_{i j ; 0} t_{00} \beta \\
& +2 \mathcal{L}_{\hat{X}} t_{j} \beta^{3} y_{i}+2 \mathcal{L}_{\hat{X}} t_{j} \beta^{3} y_{j}+4 \mathcal{L}_{\hat{X}} a_{i j} \beta^{3} t_{0}+4 \mathcal{L}_{\hat{X}} a_{i j} \beta^{2} s_{0}^{2} \\
& +4 a_{i j} \mathcal{L}_{\hat{X}} q_{00} \beta^{2}-12 e_{00}^{2} \mathcal{L}_{\hat{X}} b_{i} b_{j}-12 e_{00}^{2} \mathcal{L}_{\hat{X}} b_{j} b_{i} \\
& -2 e_{00} \mathcal{L}_{\hat{X}} e_{i j} \beta^{2}-4 e_{i 0} \mathcal{L}_{\hat{X}} e_{j 0} \beta^{2}-2 e_{i 0 ; 0} \mathcal{L}_{\hat{X}} b_{j} \beta^{2} \\
& +8 e_{00} e_{j 0} \mathcal{L}_{\hat{X}} y_{i}+8 e_{00} \mathcal{L}_{\hat{X}} e_{i 0} y_{j}+8 e_{00} \mathcal{L}_{\hat{X}} e_{j 0} y_{i} \\
& -2 e_{00 ; 0} \mathcal{L}_{\hat{X}} a_{i j} \beta+2 e_{00 ; 0} \mathcal{L}_{\hat{X}} b_{i} y_{j}+2 e_{00 ; 0} \mathcal{L}_{\hat{X}} b_{j} y_{i} \\
& +2 e_{00 ; 0} \mathcal{L}_{\hat{X}} y_{i} b_{j}+2 e_{00 ; 0} \mathcal{L}_{\hat{X}} y_{j} b_{i}+8 e_{i 0} \mathcal{L}_{\hat{X}} e_{00} y_{j} \\
& -2 e_{i j} \mathcal{L}_{\hat{X}} e_{00} \beta^{2}-4 e_{j 0} \mathcal{L}_{\hat{X}} e_{i 0} \beta^{2}-2 e_{j 0 ; 0} \mathcal{L}_{\hat{X}} b_{i} \beta^{2} \\
& +4 \mathcal{L}_{\hat{X}} a_{i j} \beta^{2} q_{00}-2 \mathcal{L}_{\hat{X}} e_{i 0 ; 0} \beta^{2} b_{j}-2 \mathcal{L}_{\hat{X}} e_{j 0 ; 0} \beta^{2} b_{i} \\
& +4 \mathcal{L}_{\hat{X}} q_{0 i} \beta^{2} y_{j}+4 \mathcal{L}_{\hat{X}} q_{0 j} \beta^{2} y_{i}-4 \mathcal{L}_{\hat{X}} q_{i 0} \beta^{2} y_{j}-4 \mathcal{L}_{\hat{X}} q_{j 0} \beta^{2} y_{i} \\
& +2 \mathcal{L}_{\hat{X}} y_{i} \beta^{3} t_{j}+2 \mathcal{L}_{\hat{X}} y_{j} \beta^{3} t_{i}-4 e_{i 0 ; 0} \mathcal{L}_{\hat{X}} y_{j} \beta \\
& -4 e_{j 0 ; 0} \mathcal{L}_{\hat{X}} y_{i} \beta+8 e_{j 0} \mathcal{L}_{\hat{X}} e_{00} y_{i}+2 \mathcal{L}_{\hat{X}} e_{00 ; 0} b_{i} y_{j} \\
& +2\left(\mathcal{L}_{\hat{X}} e_{00}\right)_{; 0} b_{j} y_{i}-4 \mathcal{L}_{\hat{X}} e_{i 0 ; 0} \beta y_{j}-4 \mathcal{L}_{\hat{X}} e_{j 0 ; 0} \beta y_{i} \\
& -8 \mathcal{L}_{\hat{X}} q_{00} y_{i} y_{j}-8 \mathcal{L}_{\hat{X}} y_{i} q_{00} y_{j}-8 \mathcal{L}_{\hat{X}} y_{j} q_{00} y_{i}+4 \mathcal{L}_{\hat{X}} y_{i} \beta^{2} q_{0 j} \\
& -4 \mathcal{L}_{\hat{X}} y_{i} \beta^{2} q_{j 0}-8 \mathcal{L}_{\hat{X}} y_{i} s_{0}^{2} y_{j}+4 \mathcal{L}_{\hat{X}} y_{j} \beta^{2} q_{0 i} \\
& -4 \mathcal{L}_{\hat{X}} y_{j} \beta^{2} q_{i 0}-8 \mathcal{L}_{\hat{X}} y_{j} s_{0}^{2} y_{i}+8 a_{i j} e_{00} \mathcal{L}_{\hat{X}} e_{00} \\
& -2 a_{i j} \mathcal{L}_{\hat{X}} e_{00 ; 0} \beta+8 e_{00} e_{i 0} \mathcal{L}_{\hat{X}} y_{j}+16 e_{00} \mathcal{L}_{\hat{X}} b_{i} \beta b_{j} s_{0} \\
& +16 e_{00} \mathcal{L}_{\hat{X}} b_{j} \beta b_{i} s_{0}+16 e_{00} \mathcal{L}_{\hat{X}} s_{0} \beta b_{i} b_{j}-16 e_{00} \beta b_{i} b_{j} \eta \\
& +12 t_{00} \beta b_{i} s_{j} t_{0}+12 t_{00} \beta b_{j} s_{i} t_{0}+16 \mathcal{L}_{\hat{X}} e_{00} \beta b_{i} b_{j} s_{0} \\
& +8 \mathcal{L}_{\hat{X}} \beta \beta s_{i} t_{0} y_{j}+8 \mathcal{L}_{\hat{X}} \beta \beta s_{j} t_{0} y_{i}-8 \mathcal{L}_{\hat{X}} s_{0} \beta b_{i} s_{0} y_{j} \\
& -8 \mathcal{L}_{\hat{X}} s_{0} \beta b_{j} s_{0} y_{i}+36 e_{i 0} \mathcal{L}_{\hat{X}} \beta \beta b_{j} s_{0}+36 e_{j 0} \mathcal{L}_{\hat{X}} \beta \beta b_{i} s_{0} \\
& -24 \mathcal{L}_{\hat{X}} \beta \beta s_{0} s_{i} y_{j}-24 \mathcal{L}_{\hat{X}} \beta \beta s_{0} s_{j} y_{i}-20 t_{00} \beta b_{i} b_{j} t_{0}-30 t_{00} \beta b_{i} s_{0} s_{j}
\end{aligned}
$$




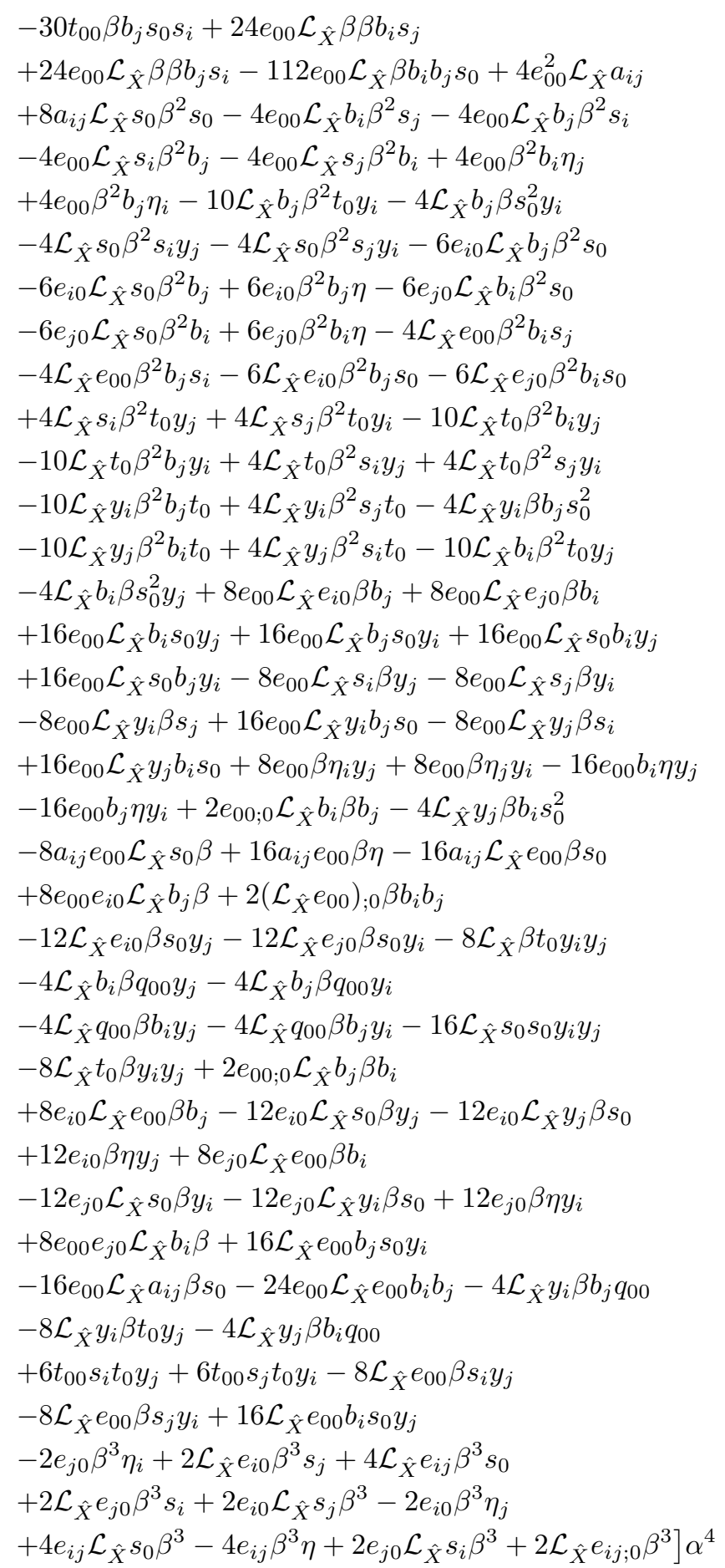




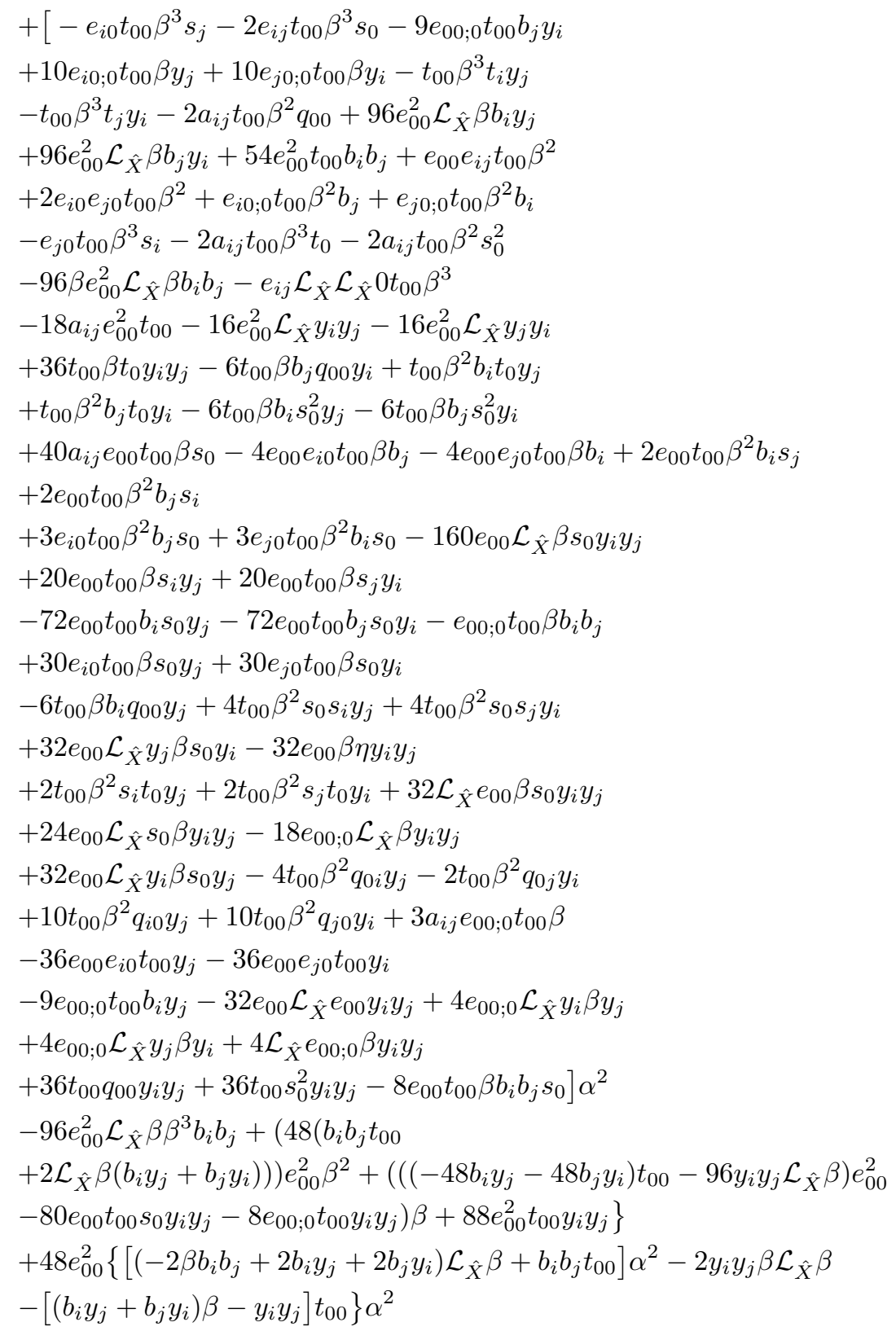

By above equation, for any point $x \in M$, the irreducible polynomial $\left(\alpha^{2}-\beta^{2}\right)$ divides $e_{00}$, or irreducible polynomial $\left(\alpha^{2}-\beta^{2}\right)$ divides last equation. In the first case, for a function $c \in C^{\infty}(M), e_{00}=2 c(x)\left(\alpha^{2}-\beta^{2}\right)$ which means that $F$ is of isotropic $S$-curvature. In the second case, the irreducible polynomial $\left(\alpha^{2}-\beta^{2}\right)$ 
divides:

$$
\begin{gathered}
{\left[\left(-2 \beta b_{i} b_{j}+2 b_{i} y_{j}+2 b_{j} y_{i}\right) \mathcal{L}_{\hat{X}} \beta+b_{i} b_{j} t_{00}\right] \alpha^{2}} \\
-2 y_{i} y_{j} \beta \mathcal{L}_{\hat{X}} \beta-\left[\left(b_{i} y_{j}+b_{j} y_{i}\right) \beta-y_{i} y_{j}\right] t_{00}
\end{gathered}
$$

By contracting previous equation with $b^{i} b^{j}$ we obtain

$$
\left(\alpha^{2} b^{4}+\beta^{2}\right) \mathcal{L}_{\hat{X}}\left(\alpha^{2}-\beta^{2}\right)+2 b^{2}\left(\alpha^{2} \mathcal{L}_{\hat{X}} \beta^{2}-\beta^{2} \mathcal{L}_{\hat{X}} \alpha^{2}\right)=\eta(x, y)\left(\alpha^{2}-\beta^{2}\right)
$$

where $\eta(x, y)$ is a quadratic form.

By $\operatorname{Irrat}=0$, then $\alpha^{2}$ divides $A_{1}$, where

$$
A_{1}=\frac{1}{2} \beta t_{00} y_{i} y_{j}\left(-192 e_{00} \beta s_{0}+96 e_{00}^{2}-19 e_{00 ; 0} \beta\right)
$$

In this case, $\mathcal{L}_{\hat{X}} \alpha^{2}=\sigma(x) \alpha^{2}$ which means that $\hat{X}$ is a Killing vector field of $\alpha$, or $\alpha^{2}$ divides $-192 e_{00} \beta s_{0}+96 e_{00}^{2}-19 e_{00 ; 0} \beta$. We have

$$
-192 e_{00} \beta s_{0}+96 e_{00}^{2}-19 e_{00 ; 0} \beta=\lambda(x, y) \alpha^{2}
$$

where $\lambda(x, y)$ is a quadratic form.

Example 4.1. Let $M=\mathbb{R}^{2}$ and $F(x, y, u, v)=\sqrt{u^{2}+v^{2}}+a u+b v$, as it defined in Example 4.1. Then by the same argument, one can see the condition 1, 2 and 3 of Theorem 1.2 are held. If we substitute $\mathcal{L}_{\hat{X}} \alpha^{2}=2 \alpha^{2}$ and $\mathcal{L}_{\hat{X}} \beta=\beta$ in 4 we have:

$$
\left[\left(\alpha^{2} b^{4}+\beta^{2}\right)\left(2 \alpha^{2}-\beta^{2}\right)=\eta^{2}(x, y)\left(\alpha^{2}-\beta^{2}\right)\right.
$$

which means that $\eta(x, y)=2\left(\alpha^{2} b^{4}+\beta^{2}\right)$. In this case, all of the conditions Theorem 2.1 hold.

Here we give an example which satisfies condition 1 and 3 of Theorem 2.1.

Example 4.2. Let $(\theta, M)$ be the Funk metric as Example 3.2, i. e. condition 1 and 3 of Theorem 1.2 are held. If we substitute (3.8) in condition 4, we have

$\frac{|y|^{2}}{1-|x|^{2}}\left\{48|y|^{2}\left(1-|x|^{2}\right)+19<x, y>^{2}\right\}=\left(|y|^{2}\left(1-|x|^{2}\right)+<x, y>^{2}\right) \lambda(x, y)$

but $\lambda(x, y)$ should be a polynomial of degree 2, which is a contradiction. Now we consider condition 2. Since $\mathcal{L}_{\hat{V}} \alpha^{2}=0$, condition 2 is reduced to

$$
\left(2 b^{2} \alpha^{2}-b^{4} \alpha^{2}-b e t a^{2}\right) \mathcal{L}_{\hat{V}} \beta^{2}=\eta(x, y)\left(\alpha^{2}-\beta^{2}\right)
$$

Then by substituting $b^{2}=|x|^{2}$ and $\mathcal{L}_{\hat{V}} \beta^{2}$ we have

$$
\begin{aligned}
\left\{\left(\frac{|x|^{2}|y|^{2}}{1-|x|^{2}}+\right.\right. & \left.\left.\frac{|x|^{2}<x, y>^{2}}{\left(1-|x|^{2}\right)^{2}}\right)\left(2-|x|^{2}\right)-\frac{<x, y>^{2}}{\left(1-|x|^{2}\right)^{2}}\right\} \\
& \times(-2<x, y><a, y>)=|y|^{2} \eta(x, y)
\end{aligned}
$$


where $\eta$ is a polynomial of degree two. If we compute the extended form of (4.5) we have

$$
a_{1} x_{1}\left(x_{1}^{2}-3 x_{2}^{2}+a_{2} x_{2}\left(x_{2}^{2}-3 x_{1}^{2}\right)=0\right.
$$

which means that $a=0$, which is a contradiction. Therefore, there is not any $\eta(x, y)$ to applies condition 2.

Here we make an example which satisfies condition 2 and 3 of Theorem 1.2.

Example 4.3. We consider Theorem 1.2 for Bergman metric. As we see in Example 3.3, conditions 2 and 3 are established automatically for a Killing vector field of $F$ and the condition 1 of Theorem 1.1 do not hold. Then by substituting (3.10) in condition 4, we find out that the term

$$
\epsilon \beta^{2}\left(77 f_{0}\left(\beta+\epsilon f_{0}\right)-19 f_{0}+96 \epsilon\left(\beta+\epsilon f_{0}\right)^{2}\right)
$$

a should be a multiple of $\alpha^{2}$, which is impossible. Thus condition 4 , does not exist.

\section{C-projective vector fields of Funk type Finsler metrics}

Let $M$ be a bounded convex in $\mathbb{R}^{n}$ and $\theta$ be the Funk type Finsler metric on $M$, i.e.,

$$
\theta_{x^{k}}=\theta \theta_{y^{k}}
$$

The spray of a Funk type Finsler metric is given by $G=y^{i} \frac{\partial}{\partial x^{i}}-2 G^{i} \frac{\partial}{\partial y^{i}}$ where $G^{i}=\frac{1}{2} \theta y^{i}$. Thus, every Funk type Finsler metric is locally projectively flat. It is easy to see that every Funk type Finsler metric is also of constant flag curvature $K=-\frac{1}{4}$. We are going to characterize all projective vector fields of Funk type Finsler metrics.

Theorem 5.1. Let $V=V^{i} \frac{\partial}{\partial x^{i}}$ be a vector field on $M$. Then the complete lift of $V$, i.e., $\hat{V}$ is a projective vector field of $(M, \theta)$ if and only if there is a 1-form $\eta:=\eta_{t}(x) y^{t}$ on $M$ which satiesties

$$
2 \frac{\partial^{2} V^{i}}{\partial x^{t} \partial x^{s}}=\eta_{t}(x) \delta_{s}^{i}+\eta_{s}(x) \delta^{i}{ }_{t}
$$

Proof. Suppose that $\hat{V}=V^{i} \frac{\partial}{\partial x^{i}}+y^{k} \frac{\partial V^{i}}{\partial x^{k}} \frac{\partial}{\partial y^{i}}$ is a projective vector field of $(M, \theta)$. Then by [1], $\hat{V}$ is a projective vector field if and only if there exsits a function $P(x, y)$ satisfying

$$
[\hat{V}, G]=P y^{i} .
$$

By a direct calculation, we have

$$
[\hat{V}, G]=\left(Q y^{i}-A^{i}\right) \frac{\partial}{\partial y^{i}},
$$


where $Q=\left(V^{j} \theta+y^{k} \frac{\partial V^{j}}{\partial x^{k}}\right) \theta_{j}$ and $A^{i}=y^{j} y^{k} \frac{\partial^{2} V^{i}}{\partial x^{k} \partial x^{j}}$. Comparing (5.3) with (5.4), we get

$$
Q y^{i}-A^{i}=P y^{i}
$$

By differentiating (5.5) with respect to $y^{s}$ we have

$$
2 y^{k} \frac{\partial^{2} V^{i}}{\partial x^{k} \partial x^{s}}=\left(P_{s}-Q_{s}\right) y^{i}+(P-Q) \delta_{s}^{i}
$$

Again by differentiating (5.6) with respect to $y^{t}$ we have

$$
2 \frac{\partial^{2} V^{i}}{\partial x^{t} \partial x^{s}}=\left(P_{s t}-Q_{s t}\right) y^{i}+\left(P_{s}-Q_{s}\right) \delta^{i}{ }_{t}+\left(P_{t}-Q_{t}\right) \delta^{i}{ }_{s}
$$

By differentiating (5.7) with respect to $y^{r}$ we have

$$
\left(P_{r s t}-Q_{r s t}\right) y^{i}+\left(P_{s t}-Q_{s t}\right) \delta_{r}^{i}+\left(P_{s r}-Q_{s r}\right) \delta^{i}+\left(P_{t r}-Q_{t r}\right) \delta^{i}{ }_{s}=0
$$

Let $i=r$ in (5.8), then we have $(n+1)\left(P_{s t}-Q_{s t}\right)=0$, which means that

$$
P=Q+\eta_{j}(x) y^{j},
$$

for some 1-form $\eta$ on $M$. If we put (5.9) into (5.5), we have

$$
y^{j} y^{k} \frac{\partial^{2} V^{i}}{\partial x^{k} \partial x^{j}}=\eta_{j}(x) y^{j} y^{i} .
$$

By differentiating (5.10) with respect to $y^{s}$ we have

$$
2 y^{k} \frac{\partial^{2} V^{i}}{\partial x^{k} \partial x^{s}}=\eta_{s}(x) y^{i}+\eta_{j}(x) y^{j} \delta^{i}{ }_{s}
$$

Again by differentiating (5.11) with respect to $y^{t}$ we have

$$
2 \frac{\partial^{2} V^{i}}{\partial x^{t} \partial x^{s}}=\eta_{s}(x) \delta^{i}{ }_{t}+\eta_{t}(x) \delta^{i}{ }_{s}
$$

Thus if $\hat{V}$ is a projective vector field of the Funk type metric $\theta$, then there is a 1-form $\eta_{t}(x)$ which satisfies (5.12).

The converse is trivial.

Theorem 5.2. Let $\theta$ be a Funk type metric on a bounded convex domain $M$ in $\mathbb{R}^{n}$. Then $V=V^{i} \frac{\partial}{\partial x^{i}}$ is a projective vector field of $\theta$ if and only if $V^{i}$ 's are given by

$$
V^{i}=x^{i}<a, x>+Q_{j}^{i} x^{j}+c^{i},
$$

where $a, c=\left(c^{1}, \cdots, c^{n}\right) \in \mathbb{R}^{n}$ are two fixed vectors, $\left(Q_{j}^{i}\right)$ is a fixed $n \times n$ real matrix and $\langle$. $\rangle$ is the Euclidean inner product on $\mathbb{R}^{n} .1$

$$
[\hat{V}, G]=P Y
$$

where

$$
\begin{aligned}
P= & -2<a, y>-\theta_{x^{k}}\left(x^{k}<a, x>+Q_{j}^{k} x^{j}+c^{k}\right) \\
& -\theta_{y^{k}}\left(y^{k}<a, x>+x^{k}<a, y>+Q_{j}^{k} y^{j}\right) .
\end{aligned}
$$


Proof. By [12] the maximum degree of projective vector fields is $n(n+2)$, thus the projective vector field of Funk type metrics are exactly as (5.13)

\section{Appendix 1}

$$
\begin{aligned}
& A_{0}=8 e_{00} t_{00} \beta^{2} s_{0} y_{i}-4 e_{00}^{2} t_{00} y_{i} \beta+e_{00 ; 0} t_{00} \beta^{2} y_{i} \\
& A_{1}=-2 t_{00}\left(8 e_{00} \beta s_{0} y_{i}-4 e_{00}^{2} y_{i}+e_{00 ; 0} \beta y_{i}\right) \\
& A_{2}=-4 t_{00} \beta^{3} s_{0} s_{i}+4 t_{00} \beta^{2} b_{i} s_{0}^{2}-12 A_{i 0} t_{00} \beta^{3} \\
& -64 e_{00}\left(\mathcal{L}_{\hat{X}} \beta\right) \beta s_{0} y_{i}-16 e_{00} \mathcal{L}_{\hat{X}} s_{0} \beta^{2} y_{i} \\
& -16 e_{00} \mathcal{L}_{\hat{X}} y_{i} \beta^{2} s_{0}-32 e_{00} t_{00} \beta b_{i} s_{0}+16 e_{00} \beta^{2} \eta y_{i} \\
& +20 e_{i 0} t_{00} \beta^{2} s_{0}-16 \mathcal{L}_{\hat{X}} e_{00} \beta^{2} s_{0} y_{i} \\
& -4 t_{00} \beta^{3} q_{i 0}+4 t_{00} \beta^{2} b_{i} q_{00}+16 t_{00} \beta^{2} t_{0} y_{i} \\
& +16 t_{00} \beta s 0^{2} y_{i}+24 e_{00}^{2} \mathcal{L}_{\hat{X}} \beta y_{i}+8 e_{00}^{2} \mathcal{L}_{\hat{X}} y_{i} \beta \\
& +12 e_{00}^{2} t_{00} b_{i}-8 e_{00} e_{i 0} t_{00} \beta+16 e_{00} \mathcal{L}_{\hat{X}} e_{00} \beta y_{i} \\
& -24 e_{00} t_{00} s_{0} y_{i}-8 e_{00 ; 0}\left(\mathcal{L}_{\hat{X}} \beta\right) \beta y_{i}-2 e_{00 ; 0} \mathcal{L}_{\hat{X}} y_{i} \beta^{2} \\
& -4 e_{00 ; 0} t_{00} \beta b_{i}-5 e_{00 ; i} t_{00} \beta^{2}+10 e_{i 0 ; 0} t_{00} \beta^{2} \\
& -2 \mathcal{L}_{\hat{X}} e_{00 ; 0} \beta^{2} y_{i}+16 t_{00} \beta q_{00} y_{i}-3 e_{00 ; 0} t_{00} y_{i} \\
& A_{3}=8 \mathcal{L}_{\hat{X}} y_{i} \beta^{3} t_{0}+8 \mathcal{L}_{\hat{X}} y_{i} \beta^{2} s_{0}^{2}+8 \mathcal{L}_{\hat{X}} y_{i} \beta^{2} q_{00} \\
& +16 t_{00} s_{0}^{2} y_{i}+16 t_{00} q_{00} y_{i}-2 e_{00 ; 0} \mathcal{L}_{\hat{X}} b_{i} \beta^{2} \\
& -8 e_{00} e_{i 0} t_{00}+20 e_{i 0 ; 0} \mathcal{L}_{\hat{X}} \beta \beta^{2}-24 A_{i 0} \mathcal{L}_{\hat{X}} \beta \beta^{3} \\
& -36 A_{i 0} t_{00} \beta^{2}-10 e_{00 ; i} \mathcal{L}_{\hat{X}} \beta \beta^{2}-8 e_{00 ; 0} \mathcal{L}_{\hat{X}} \beta y_{i} \\
& +20 e_{i 0 ; 0} t_{00} \beta-10 e_{00 ; i} t_{00} \beta-4 \mathcal{L}_{\hat{X}} e_{00 ; 0} y_{i} \beta \\
& -2 e_{i 0} \beta^{3} \eta+24 e_{00}^{2} \mathcal{L}_{\hat{X}} \beta b_{i}-12 t_{00} \beta^{2} q_{0 i} \\
& -4 e_{00 ; 0} t_{00} b_{i}+16 e_{00} \mathcal{L}_{\hat{X}} e_{00} y_{i}-2 \mathcal{L}_{\hat{X}} e_{00 ; 0} \beta^{2} b_{i} \\
& -20 t_{00} \beta^{2} q_{i 0}+8 e_{i 0} \mathcal{L}_{\hat{X}} s_{0} \beta^{3}+8 \mathcal{L}_{\hat{X}} e_{i 0} \beta^{3} s_{0} \\
& -4 e_{00} \mathcal{L}_{\hat{X}} e_{i 0} \beta^{2}-4 e_{i 0} \mathcal{L}_{\hat{X}} e_{00} \beta^{2}+8 \mathcal{L}_{\hat{X}} t_{0} \beta^{3} y_{i} \\
& +8 \mathcal{L}_{\hat{X}} q_{00} \beta^{2} y_{i}-4 e_{00 ; 0} \mathcal{L}_{\hat{X}} y_{i} \beta+8 e_{00}^{2} \mathcal{L}_{\hat{X}} b_{i} \beta \\
& -12 e_{00} \beta^{3} \eta_{i}-4 \mathcal{L}_{\hat{X}} A_{i 0} \beta^{4}-2 \mathcal{L}_{\hat{X}} e_{00 ; i} \beta^{3} \\
& +4 \mathcal{L}_{\hat{X}} e_{i 0 ; 0} \beta^{3}+8 e_{00}^{2} \mathcal{L}_{\hat{X}} y_{i}-64 e_{00} \mathcal{L}_{\hat{X}} \beta b_{i} s_{0} \beta \\
& +16 \mathcal{L}_{\hat{X}} s_{0} \beta^{2} s_{0} y_{i}-64 e_{00} \mathcal{L}_{\hat{X}} \beta s_{0} y_{i}-32 e_{00} t_{00} b_{i} s_{0}+40 e_{i 0} \mathcal{L}_{\hat{X}} \beta \beta^{2} s_{0} \\
& +40 e_{i 0} t_{00} \beta s_{0}-8 t_{00} \beta^{2} b_{i} t_{0} \\
& +24 t_{00} \beta b_{i} s_{0}^{2}+24 t_{00} \beta b_{i} q_{00}+16 t_{00} \beta t_{0} y_{i}+32 \mathcal{L}_{\hat{X}} \beta s_{0}^{2} y_{i} \beta \\
& +32 \mathcal{L}_{\hat{X}} \beta q_{00} y_{i} \beta+16 e_{00} \beta^{2} b_{i} \eta \\
& +32 e_{00} \beta \eta y_{i}-16 e_{00} e_{i 0} \mathcal{L}_{\hat{X}} \beta \beta-8 e_{00 ; 0} \mathcal{L}_{\hat{X}} \beta b_{i} \beta \\
& +16 e_{00} \mathcal{L}_{\hat{X}} e_{00} b_{i} \beta-32 t_{00} \beta^{2} s_{0} s i \\
& -16 e_{00} \mathcal{L}_{\hat{X}} b_{i} \beta^{2} s_{0}-32 e_{00} \mathcal{L}_{\hat{X}} y_{i} \beta s_{0}-16 e_{00} \mathcal{L}_{\hat{X}} s_{0} \beta^{2} b_{i} \\
& -32 e_{00} \mathcal{L}_{\hat{X}} s_{0} \beta y_{i} s_{0}-16 e_{00} \mathcal{L}_{\hat{X}} y
\end{aligned}
$$




$$
\begin{aligned}
& A_{4}=-8 e_{00} \mathcal{L}_{\hat{X}} e_{i 0} \beta-8 e_{i 0} \mathcal{L}_{\hat{X}} e_{00} \beta+32 \mathcal{L}_{\hat{X}} \beta s_{0}^{2} y_{i} \\
& +8 \mathcal{L}_{\hat{X}} b_{i} \beta^{2} q_{00}+16 \mathcal{L}_{\hat{X}} y_{i} \beta^{2} t_{0}+20 t_{00} b_{i} s_{0}^{2} \\
& +32 \mathcal{L}_{\hat{X}} \beta q_{00} y_{i}+20 t_{00} b_{i} q_{00}-16 e_{00} e_{i 0} \mathcal{L}_{\hat{X}} \beta \\
& -72 A_{i 0} \mathcal{L}_{\hat{X}} \beta \beta^{2}-8 e_{00 ; 0} \mathcal{L}_{\hat{X}} \beta b_{i} \\
& +\left(40 e_{i 0 ; 0} \mathcal{L}_{\hat{X}} \beta\right) \beta-36 A_{i 0} t_{00} \beta-20 e_{00 ; i}\left(\mathcal{L}_{\hat{X}} \beta\right) \beta \\
& -4 \mathcal{L}_{\hat{X}} e_{00 ; 0} b_{i} \beta-28 t_{00} q_{i 0} \beta-8 \mathcal{L}_{\hat{X}} s_{0} \beta^{3} s_{i} \\
& -36 e_{00} \beta^{2} \eta_{i}-8 \mathcal{L}_{\hat{X}} b_{i} \beta^{3} t_{0}-6 e_{i 0} \beta^{2} \eta \\
& +8 \mathcal{L}_{\hat{X}} b_{i} \beta^{2} s_{0}^{2}-24\left(\mathcal{L}_{\hat{X}} \beta\right) \beta^{2} q_{0 i}-24 t_{00} \beta q_{0 i} \\
& +16 e_{00} \mathcal{L}_{\hat{X}} e_{00} b_{i}+16 e_{00} \eta y_{i}-\left(16 \mathcal{L}_{\hat{X}} \beta\right) \beta^{2} q_{i 0} \\
& -16 e_{00} \mathcal{L}_{\hat{X}} y_{i} s_{0}+24 e_{i 0} \mathcal{L}_{\hat{X}} s_{0} \beta^{2} \\
& +24 \mathcal{L}_{\hat{X}} e_{i 0} \beta^{2} s_{0}-16 e_{00} \mathcal{L}_{\hat{X}} s_{0} y_{i}-16 \mathcal{L}_{\hat{X}} e_{00} s_{0} y_{i} \\
& +8 \mathcal{L}_{\hat{X}} t_{0} \beta^{3} b_{i}+8 \mathcal{L}_{\hat{X}} q_{00} \beta^{2} b_{i}+16 \mathcal{L}_{\hat{X}} t_{0} \beta^{2} y_{i} \\
& +20 e_{i 0} t_{00} s_{0}+16 \mathcal{L}_{\hat{X}} y_{i} s_{0}^{2} \beta+16 \mathcal{L}_{\hat{X}} y_{i} q_{00} \beta \\
& -4 e_{00 ; 0} \mathcal{L}_{\hat{X}} b_{i} \beta+16 \mathcal{L}_{\hat{X}} q_{00} y_{i} \beta-8 \mathcal{L}_{\hat{X}} s_{i} \beta^{3} s_{0} \\
& -8 \mathcal{L}_{\hat{X}} q_{i 0} \beta^{3}-16 \mathcal{L}_{\hat{X}} A_{i 0} \beta^{3}-2 e_{00 ; 0} \mathcal{L}_{\hat{X}} y_{i}+8 e_{00}^{2} \mathcal{L}_{\hat{X}} b_{i} \\
& -5 e_{00 ; i} t_{00}-2 \mathcal{L}_{\hat{X}} e_{00 ; 0} y_{i}-6 \mathcal{L}_{\hat{X}} e_{00 ; i} \beta^{2} \\
& +12 \mathcal{L}_{\hat{X}} e_{i 0 ; 0} \beta^{2}+10 e_{i 0 ; 0} t_{00}+32 \mathcal{L}_{\hat{X}} s_{0} \beta s_{0} y_{i} \\
& -64 e_{00} \mathcal{L}_{\hat{X}} \beta b_{i} s_{0}+80 e_{i 0}\left(\mathcal{L}_{\hat{X}} \beta\right) \beta s_{0}-32 t_{00} \beta b_{i} t_{0} \\
& +32 e_{00} \beta b_{i} \eta+32 \mathcal{L}_{\hat{X}} \beta b_{i} s_{0}^{2} \beta+32 \mathcal{L}_{\hat{X}} \beta b_{i} q_{00} \beta \\
& -40\left(\mathcal{L}_{\hat{X}} \beta\right) \beta^{2} s_{0} s_{i}-52 t_{00} \beta s_{0} s_{i}-32 e_{00} \mathcal{L}_{\hat{X}} b_{i} \beta s_{0} \\
& -32 e_{00} \mathcal{L}_{\hat{X}} s_{0} \beta b_{i}-32 \mathcal{L}_{\hat{X}} e_{00} \beta b_{i} s_{0} \\
& +16 \mathcal{L}_{\hat{X}} s_{0} \beta^{2} b_{i} s_{0}+48\left(\mathcal{L}_{\hat{X}} \beta\right) \beta t_{0} y_{i} \\
& A_{5}=-36 e_{00} \eta_{i} \beta-6 e_{i 0} \eta \beta+8 \mathcal{L}_{\hat{X}} y_{i} \beta t_{0}- \\
& 72 A_{i 0}\left(\mathcal{L}_{\hat{X}} \beta\right) \beta-32 \mathcal{L}_{\hat{X}} \beta q_{i 0} \beta+24 e_{i 0} \mathcal{L}_{\hat{X}} s_{0} \beta \\
& +24 \mathcal{L}_{\hat{X}} e_{i 0} s_{0} \beta+16 \mathcal{L}_{\hat{X}} b_{i} s_{0}^{2} \beta-24 \mathcal{L}_{\hat{X}} s_{i} \beta^{2} s_{0} \\
& -24 \mathcal{L}_{\hat{X}} s_{0} \beta^{2} s_{i}+32 \mathcal{L}_{\hat{X}} \beta b_{i} s_{0}^{2}-16 e_{00} \mathcal{L}_{\hat{X}} s_{0} b_{i} \\
& +32 \mathcal{L}_{\hat{X}} \beta b_{i} q_{00}-48\left(\mathcal{L}_{\hat{X}} \beta\right) \beta q_{0 i}+16 e_{00} b_{i} \eta \\
& -24 t_{00} b_{i} t_{0}-24 t_{00} s_{0} s_{i}-16 e_{00} \mathcal{L}_{\hat{X}} b_{i} s_{0} \\
& -16 \mathcal{L}_{\hat{X}} e_{00} b_{i} s_{0}+16 \mathcal{L}_{\hat{X}} t_{0} \beta^{2} b_{i}+8 \mathcal{L}_{\hat{X}} \beta t_{0} y_{i} \\
& +16\left(\mathcal{L}_{\hat{X}} s_{0}\right) s_{0} y_{i}+8 \mathcal{L}_{\hat{X}} t_{0} \beta y_{i}+40 e_{i 0} \mathcal{L}_{\hat{X}} \beta s_{0} \\
& -32 \mathcal{L}_{\hat{X}} b_{i} \beta^{2} t_{0}+16 \mathcal{L}_{\hat{X}} b_{i} q_{00} \beta+16 \mathcal{L}_{\hat{X}} q_{00} b_{i} \beta \\
& -12 t_{00} q_{0 i}-4 e_{00} \mathcal{L}_{\hat{X}} e_{i 0}-4 e_{i 0} \mathcal{L}_{\hat{X}} e_{00} \\
& -24 \mathcal{L}_{\hat{X}} q_{i 0} \beta^{2}+8 \mathcal{L}_{\hat{X}} y_{i} s_{0}^{2}+8 \mathcal{L}_{\hat{X}} y_{i} q_{00}-2 e_{00 ; 0} \mathcal{L}_{\hat{X}} b_{i} \\
& +8 \mathcal{L}_{\hat{X}} q_{00} y_{i}-10 e_{00 ; i} \mathcal{L}_{\hat{X}} \beta \\
& -2 \mathcal{L}_{\hat{X}} e_{00 ; 0} b_{i}-12 t_{00} q_{i 0}-6 \mathcal{L}_{\hat{X}} e_{00 ; i} \beta+12 \mathcal{L}_{\hat{X}} e_{i 0 ; 0} \beta \\
& -24 \mathcal{L}_{\hat{X}} A_{i 0} \beta^{2}+20 e_{i 0 ; 0} \mathcal{L}_{\hat{X}} \beta-12 A_{i 0} t_{00}
\end{aligned}
$$




$$
\begin{aligned}
& \left.-80 \mathcal{L}_{\hat{X}} \beta\right) \beta s_{0} s_{i}-32 \mathcal{L}_{\hat{X}} \beta b_{i} t_{0} \beta+32 \mathcal{L}_{\hat{X}} s_{0} \beta b_{i} s_{0} \\
A_{6}= & -32 \mathcal{L}_{\hat{X}} \beta b_{i} t_{0}-40 \mathcal{L}_{\hat{X}} \beta s_{0} s_{i}-40 \mathcal{L}_{\hat{X}} b_{i} \beta t_{0} \\
& +8 \mathcal{L}_{\hat{X}} b_{i} s_{0}^{2}-24 \mathcal{L}_{\hat{X}} s_{0} \beta s_{i}+16 \mathcal{L}_{\hat{X}} s_{0} b_{i} s_{0} \\
& -24 \mathcal{L}_{\hat{X}} s_{i} \beta s_{0}+8 \mathcal{L}_{\hat{X}} t_{0} \beta b_{i}-24 A_{i 0} \mathcal{L}_{\hat{X}} \beta \\
& -12 e_{00} \eta_{i}+8 e_{i 0} \mathcal{L}_{\hat{X}} s_{0}-2 e_{i 0} \eta-16 \mathcal{L}_{\hat{X}} A_{i 0} \beta \\
& +8 \mathcal{L}_{\hat{X}} e_{i 0} s_{0}-24 \mathcal{L}_{\hat{X}} \beta q_{0 i}-16 \mathcal{L}_{\hat{X}} \beta q_{i 0}+8 \mathcal{L}_{\hat{X}} b_{i} q_{00}+8 \mathcal{L}_{\hat{X}} q_{00} b_{i} \\
& -24 \mathcal{L}_{\hat{X}} q_{i 0} \beta-2 \mathcal{L}_{\hat{X}} e_{00 ; i}+4 \mathcal{L}_{\hat{X}} e_{i 0 ; 0} \\
A_{7}= & -16 \mathcal{L}_{\hat{X}} b_{i} t_{0}-8 \mathcal{L}_{\hat{X}} s_{0} s_{i}-8 \mathcal{L}_{\hat{X}} s_{i} s_{0}-4 \mathcal{L}_{\hat{X}} A_{i 0}-8 \mathcal{L}_{\hat{X}} q_{i 0}
\end{aligned}
$$

\section{Appendix 2}

$$
\begin{aligned}
& A_{0}=\frac{1}{2} t_{00} \beta^{2} y_{i} y_{j}\left(-32 e_{00} \beta s_{0}+16 e_{00}^{2}-3 e_{00 ; 0} \beta\right) \\
& A_{1}=\frac{1}{2} \beta t_{00} y_{i} y_{j}\left(-192 e_{00} \beta s_{0}+96 e_{00}^{2}-19 e_{00 ; 0} \beta\right) \\
& A_{2}=8 a_{i j} e_{00} t_{00} \beta^{3} s_{0}+28 e_{00}^{2} \mathcal{L}_{\hat{X}} \beta \beta y_{i} y_{j}-4 e_{00 ; 0} \mathcal{L}_{\hat{X}} \beta \beta^{2} y_{i} y_{j} \\
& +2 t_{00} \beta^{4} s_{0} s_{i} y_{j}+2 t_{00} \beta^{4} s_{0} s_{j} y_{i} \\
& -2 t_{00} \beta^{3} b_{i} s_{0}^{2} y_{j}-2 t_{00} \beta^{3} b_{j} s_{0}^{2} y_{i}+6 e_{j 0} t_{00} \beta^{3} s_{0} y_{i} \\
& +4 e_{00} t_{00} \beta^{3} s_{i} y_{j}+4 e_{00} t_{00} \beta^{3} s_{j} y_{i}+6 e_{i 0} t_{00} \beta^{3} s_{0} y_{j} \\
& +8 e_{00} \mathcal{L}_{\hat{X}} y_{j} \beta^{3} s_{0} y_{i}+12 e_{00}^{2} t_{00} b_{i} y_{j} \beta+12 e_{00}^{2} t_{00} b_{j} y_{i} \beta \\
& -8 e_{00} \beta^{3} \eta y_{i} y_{j}-2 t_{00} \beta^{3} b_{i} q_{00} y_{j} \\
& -2 t_{00} \beta^{3} b_{j} q_{00} y_{i}-8 e_{00} e_{i 0} t_{00} \beta^{2} y_{j}-8 e_{00} e_{j 0} t_{00} \beta^{2} y_{i} \\
& -2 e_{00 ; 0} t_{00} \beta^{2} b_{i} y_{j}-2 e_{00 ; 0} t_{00} \beta^{2} b_{j} y_{i} \\
& +8 \mathcal{L}_{\hat{X}} e_{00} \beta^{3} s_{0} y_{i} y_{j}-8 e_{00} \mathcal{L}_{\hat{X}} e_{00} \beta^{2} y_{i} y_{j} \\
& +4 e_{00} \mathcal{L}_{\hat{X}} s_{0} \beta^{3} y_{i} y_{j}+8 e_{00} \mathcal{L}_{\hat{X}} y_{i} \beta^{3} s_{0} y_{j}-4 a_{i j} e_{00}^{2} t_{00} \beta^{2} \\
& +(1 / 2) a_{i j} e_{00 ; 0} t_{00} \beta^{3}+2 e_{i 0 ; 0} t_{00} \beta^{3} y_{j}+2 e_{j 0 ; 0} t_{00} \beta^{3} y_{i} \\
& +2 t_{00} \beta^{4} q_{i 0} y_{j}+2 t_{00} \beta^{4} q_{j 0} y_{i}-4 e_{00}^{2} \mathcal{L}_{\hat{X}} y_{i} \beta^{2} y_{j} \\
& -4 e_{00}^{2} \mathcal{L}_{\hat{X}} y_{j} \beta^{2} y_{i}+e_{00 ; 0} \mathcal{L}_{\hat{X}} y_{i} \beta^{3} y_{j}+e_{00 ; 0} \mathcal{L}_{\hat{X}} y_{j} \beta^{3} y_{i} \\
& +\left(\mathcal{L}_{\hat{X}} e_{00}\right)_{; 0} \beta^{3} y_{i} y_{j}+8 t_{00} \beta^{3} t 0 y_{i} y_{j} \\
& +8 t_{00} \beta^{2} q_{00} y_{i} y_{j}+8 t_{00} \beta^{2} s_{0}^{2} y_{i} y_{j}+88 e_{00}^{2} t_{00} y_{i} y_{j} \\
& -16 e_{00} t_{00} \beta^{2} b_{i} s_{0} y_{j}-16 e_{00} t_{00} \beta^{2} b_{j} s_{0} y_{i} \\
& -200 e_{00} t_{00} \beta s_{0} y_{i} y_{j}-48 e_{00} \mathcal{L}_{\hat{X}} \beta \beta^{2} s_{0} y_{i} y_{j}-\left(43 / 2 e_{00 ; 0}\right) t_{00} \beta y_{i} y_{j} \\
& A_{3}=-e_{i j ; 0} t_{00} \beta^{4}-8 t_{00} \beta^{2} b_{i} q_{00} y_{j}-8 t_{00} \beta^{2} b_{j} q_{00} y_{i}+6 t_{00} \beta^{3} s_{0} s_{i} y_{j} \\
& +6 t_{00} \beta^{3} s_{0} s_{j} y_{i}-8 t_{00} \beta^{2} b_{i} s_{0}^{2} y_{j} \\
& -8 t_{00} \beta^{2} b_{j} s_{0}^{2} y_{i}+48 a_{i j} e_{00} t_{00} \beta^{2} s_{0}-4 e_{00} e_{i 0} t_{00} \beta^{2} b_{j} \\
& -4 e_{00} e_{j 0} t_{00} \beta^{2} b_{i}+44 t_{00} \beta^{2} t_{0} y_{i} y_{j} \\
& +44 t_{00} \beta s_{0}^{2} y_{i} y_{j}-44 e_{00} e_{i 0} t_{00} \beta y_{j}-44 e_{00} e_{j 0} t_{00} \beta y_{i} \\
& -22 e_{00 ; 0} \mathcal{L}_{\hat{X}} \beta \beta y_{i} y_{j}-11 e_{00 ; 0} t_{00} \beta b_{i} y_{j}
\end{aligned}
$$




$$
\begin{aligned}
& -11 e_{00 ; 0} t_{00} \beta b_{j} y_{i}+44 t_{00} \beta q_{00} y_{i} y_{j}+24 e_{00} t_{00} \beta^{2} s_{i} y_{j} \\
& +24 e_{00} t_{00} \beta^{2} s_{j} y_{i}+36 e_{i 0} t_{00} \beta^{2} s_{0} y_{j} \\
& +36 e_{j 0} t_{00} \beta^{2} s_{0} y_{i}+t_{00} \beta^{3} b_{i} t_{0} y_{j}+t_{00} \beta^{3} b_{j} t_{0} y_{i} \\
& -e_{00 ; 0} t_{00} \beta^{2} b_{i} b_{j}+2 e_{00} t_{00} \beta^{3} b_{i} s_{j}+2 e_{00} t_{00} \beta^{3} b_{j} s_{i} \\
& +3 e_{i 0} t_{00} \beta^{3} b_{j} s_{0}+3 e_{j 0} t_{00} \beta^{3} b_{i} s_{0}+40 e_{00} \mathcal{L}_{\hat{X}} y_{j} \beta^{2} s_{0} y_{i} \\
& -40 e_{00} \beta^{2} \text { eta }_{i} y_{j}+2 t_{00} \beta^{3} s_{i} t_{0} y_{j} \\
& +2 t_{00} \beta^{3} s_{j} t_{0} y_{i}+6 e_{00}^{2} t_{00} b_{i} b_{j} \beta+40 \mathcal{L}_{\hat{X}} e_{00} \beta^{2} s_{0} y_{i} y_{j} \\
& -40 e_{00} \mathcal{L}_{\hat{X}} e_{00} \beta y_{i} y_{j}+28 e_{00} \mathcal{L}_{\hat{X}} s_{0} \beta^{2} y_{i} y_{j} \\
& +40 e_{00} \mathcal{L}_{\hat{X}} y_{i} \beta^{2} s_{0} y_{j}-2 t_{00} \beta^{3} q_{0 j} y_{i}-e_{j 0} t_{00} \beta^{4} s_{i} \\
& +2 e_{i 0} e_{j 0} t_{00} \beta^{3}+e_{00} e_{i j} t_{00} \beta^{3}-e_{i 0} t_{00} \beta^{4} s_{0} \\
& -2 e_{i j} t_{00} \beta^{4} s_{0}-t_{00} \beta^{4} t_{i} y_{j}-t_{00} \beta^{4} t_{j} y_{i}+e_{i 0 ; 0} t_{00} \beta^{3} b_{j} \\
& +e_{j 0 ; 0} t_{00} \beta^{3} b_{i}-4 t_{00} \beta^{3} q_{0 i} y_{j}-2 a_{i j} t_{00} \beta^{3} q_{00} \\
& -2 a_{i j} t_{00} \beta^{4} t_{0}-2 a_{i j} t_{00} \beta^{3} s_{0}^{2}+12 t_{00} \beta^{3} q_{i 0} y_{j} \\
& +12 t_{00} \beta^{3} q_{j 0} y_{i}-22 a_{i j} e_{00}^{2} t_{00} \beta+124 e_{00}^{2} \mathcal{L}_{\hat{X}} \beta y_{i} y_{j} \\
& +60 e_{00}^{2} t_{00} b_{i} y_{j}+60 e_{00}^{2} t_{00} b_{j} y_{i}-20 e_{00}^{2} \mathcal{L}_{\hat{X}} y_{i} \beta y_{j} \\
& -20 e_{00}^{2} \mathcal{L}_{\hat{X}} y_{j} \beta y_{i}+5 e_{00 ; 0} \mathcal{L}_{\hat{X}} y_{i} \beta^{2} y_{j} \\
& +5 e_{00 ; 0} \mathcal{L}_{\hat{X}} y_{j} \beta^{2} y_{i}+5\left(\mathcal{L}_{\hat{X}} e_{00}\right)_{; 0} \beta^{2} y_{i} y_{j} \\
& +\left(7 / 2 a_{i j}\right) e_{00 ; 0} t_{00} \beta^{2}-120 e_{00} t_{00} s_{0} y_{i} y_{j} \\
& -8 e_{00} t_{00} \beta^{2} b_{i} b_{j} s_{0}-208 e_{00} \mathcal{L}_{\hat{X}} \beta \beta s_{0} y_{i} y_{j}-88 e_{00} t_{00} \beta b_{i} s_{0} y_{j} \\
& -88 e_{00} t_{00} \beta b_{j} s_{0} y_{i}+12 e_{i 0 ; 0} t_{00} \beta^{2} y_{j} \\
& +12 e_{j 0 ; 0} t_{00} \beta^{2} y_{i}-\left(27 / 2 e_{00 ; 0}\right) t_{00} y_{i} y_{j} \\
& A_{4}=-9 e_{i j ; 0} t_{00} \beta^{3}-18 a_{i j} e_{00}^{2} t_{00}-2 \mathcal{L}_{\hat{X}} q_{i 0} \beta^{4} y_{j} \\
& -2 \mathcal{L}_{\hat{X}} q_{j 0} \beta^{4} y_{i}-2 e_{i 0 ; 0} \mathcal{L}_{\hat{X}} y_{j} \beta^{3}-2 e_{j 0 ; 0} \mathcal{L}_{\hat{X}} y_{i} \beta^{3} \\
& -2\left(\mathcal{L}_{\hat{X}} e_{i 0}\right)_{; 0} \beta^{3} y_{j}-2 \mathcal{L}_{\hat{X}} e_{j 0 ; 0} \beta^{3} y_{i}-16 e_{00}^{2} \mathcal{L}_{\hat{X}} y_{i} y_{j} \\
& -16 e_{00}^{2} \mathcal{L}_{\hat{X}} y_{j} y_{i}-2 \mathcal{L}_{\hat{X}} y_{i} \beta^{4} q_{j 0}-2 \mathcal{L}_{\hat{X}} y_{j} \beta^{4} q_{i 0} \\
& -a_{i j}\left(\mathcal{L}_{\hat{X}} e_{00}\right)_{; 0} \beta^{3}+4 e_{00}^{2} \mathcal{L}_{\hat{X}} a_{i j} \beta^{2}-e_{00 ; 0} \mathcal{L}_{\hat{X}} a_{i j} \beta^{3} \\
& +17 t_{00} \beta^{2} b_{i} t_{0} y_{j}+17 t_{00} \beta^{2} b_{j} t_{0} y_{i}+18 t_{00} \beta b_{i} s_{0}^{2} y_{j} \\
& +18 t_{00} \beta b_{j} s_{0}^{2} y_{i}+72 a_{i j} e_{00} t_{00} \beta s_{0}-56 e_{00} e_{i 0} \mathcal{L}_{\hat{X}} \beta \beta y_{j} \\
& -36 e_{00} e_{i 0} t_{00} \beta b_{j}-56 e_{00} e_{j 0} \mathcal{L}_{\hat{X}} \beta \beta y_{i} \\
& -36 e_{00} e_{j 0} t_{00} \beta b_{i}+18 e_{00} t_{00} \beta^{2} b_{i} s_{j}+18 e_{00} t_{00} \beta^{2} b_{j} s_{i} \\
& +36 e_{i 0} \mathcal{L}_{\hat{X}} \beta \beta^{2} s_{0} y_{j}+27 e_{i 0} t_{00} \beta^{2} b_{j} s_{0} \\
& +36 e_{j 0} \mathcal{L}_{\hat{X}} \beta \beta^{2} s_{0} y_{i}+27 e_{j 0} t_{00} \beta^{2} b_{i} s_{0}-12 \mathcal{L}_{\hat{X}} \beta \beta^{2} b_{i} q_{00} y_{j} \\
& -12 \mathcal{L}_{\hat{X}} \beta \beta^{2} b_{j} q_{00} y_{i}+48 \mathcal{L}_{\hat{X}} \beta \beta^{2} t_{0} y_{i} y_{j} \\
& +56 \mathcal{L}_{\hat{X}} \beta \beta s_{0}^{2} y_{i} y_{j}-160 e_{00} \mathcal{L}_{\hat{X}} \beta s_{0} y_{i} y_{j}+36 e_{00} t_{00} \beta s_{i} y_{j} \\
& +36 e_{00} t_{00} \beta s_{j} y_{i}-72 e_{00} t_{00} b_{i} s_{0} y_{j} \\
& -72 e_{00} t_{00} b_{j} s_{0} y_{i}-14 e_{00 ; 0} \mathcal{L}_{\hat{X}} \beta \beta b_{i} y_{j}-14 e_{00 ; 0} \mathcal{L}_{\hat{X}} \beta \beta b_{j} y_{i}
\end{aligned}
$$




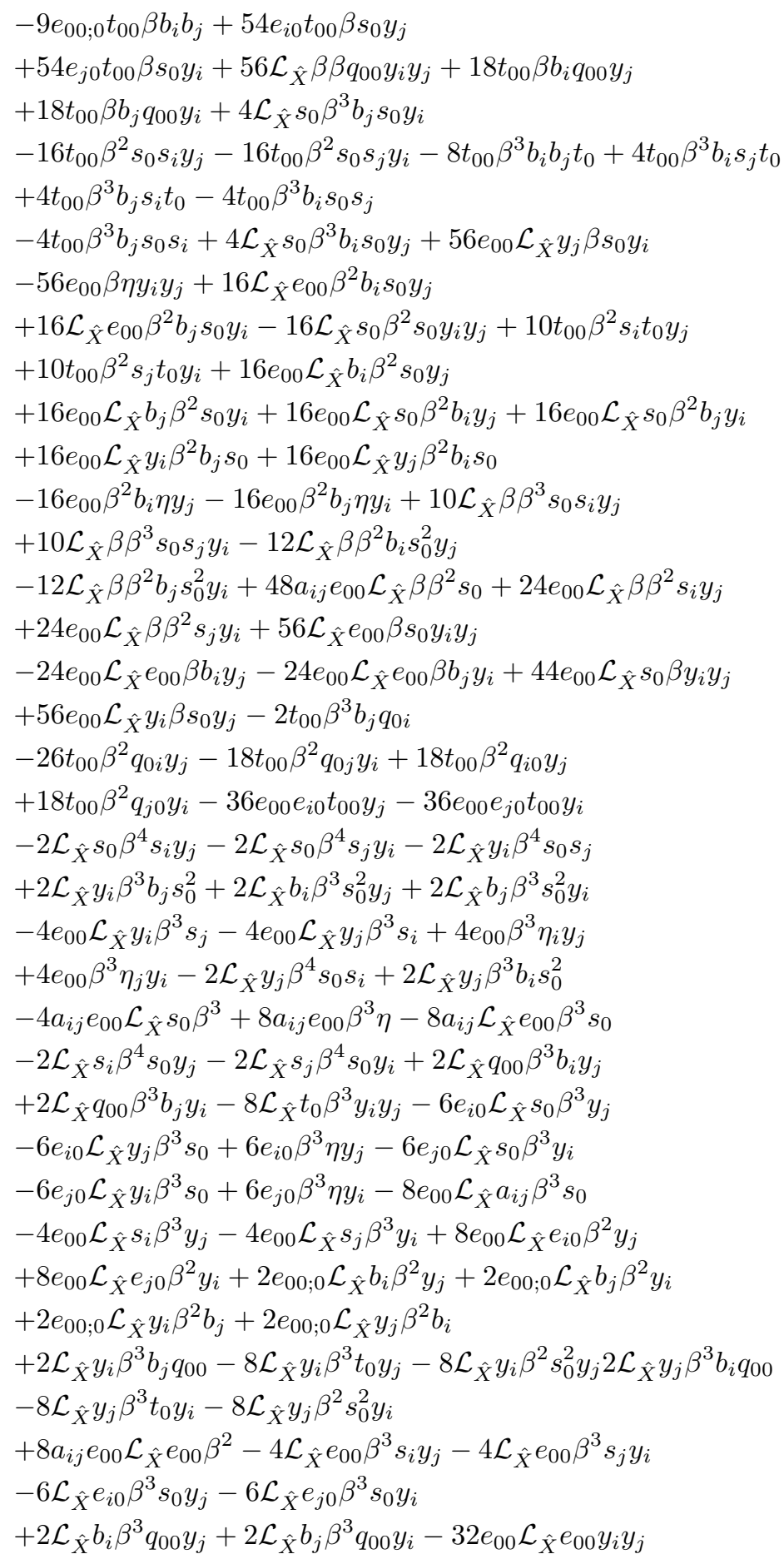




$$
\begin{aligned}
& +7 e_{00 ; 0} \mathcal{L}_{\hat{X}} y_{i} \beta y_{j}+7 e_{00 ; 0} \mathcal{L}_{\hat{X}} y_{j} \beta y_{i} \\
& +7 \mathcal{L}_{\hat{X}} e_{00 ; 0} \beta y_{i} y_{j}+8 e_{i 0} \mathcal{L}_{\hat{X}} e_{00} \beta^{2} y_{j}+8 e_{j 0} \mathcal{L}_{\hat{X}} e_{00} \beta^{2} y_{i} \\
& +2 \mathcal{L}_{\hat{X}} e_{00 ; 0} \beta^{2} b_{i} y_{j}+2 \mathcal{L}_{\hat{X}} e_{00 ; 0} \beta^{2} b_{j} y_{i} \\
& -8 \mathcal{L}_{\hat{X}} q_{00} \beta^{2} y_{i} y_{j}-8 \mathcal{L}_{\hat{X}} y_{i} \beta^{2} q_{00} y_{j}-8 \mathcal{L}_{\hat{X}} y_{j} \beta^{2} q_{00} y_{i} \\
& -12 e_{00}^{2} \mathcal{L}_{\hat{X}} b_{i} \beta y_{j}-12 e_{00}^{2} \mathcal{L}_{\hat{X}} b_{j} \beta y_{i} \\
& -12 e_{00}^{2} \mathcal{L}_{\hat{X}} y_{i} \beta b_{j}-12 e_{00}^{2} \mathcal{L}_{\hat{X}} y_{j} \beta b_{i}+8 e_{00} e_{i 0} \mathcal{L}_{\hat{X}} y_{j} \beta^{2} \\
& +8 e_{00} e_{j 0} \mathcal{L}_{\hat{X}} y_{i} \beta^{2}+\left(11 / 2 a_{i j}\right) e_{00 ; 0} t_{00} \beta \\
& +36 t_{00} \beta t_{0} y_{i} y_{j}+36 t_{00} q_{00} y_{i} y_{j}+36 t_{00} s_{0}^{2} y_{i} y_{j}-112 e_{00} \mathcal{L}_{\hat{X}} \beta \beta b_{i} s_{0} y_{j} \\
& -112 e_{00} \mathcal{L}_{\hat{X}} \beta \beta b_{j} s_{0} y_{i} \\
& -72 e_{00} t_{00} \beta b_{i} b_{j} s_{0}-9 e_{i 0} t_{00} \beta^{3} s_{j}-18 e_{i j} t_{00} \beta^{3} s_{0} \\
& +10 \mathcal{L}_{\hat{X}} \beta \beta^{3} q_{i 0} y_{j}+10 \mathcal{L}_{\hat{X}} \beta \beta^{3} q_{j 0} y_{i}-18 a_{i j} t_{00} \beta^{3} t_{0} \\
& -18 a_{i j} t_{00} \beta^{2} s_{0}^{2}-18 e_{00 ; 0} \mathcal{L}_{\hat{X}} \beta y_{i} y_{j}-9 e_{00 ; 0} t_{00} b_{i} y_{j} \\
& -9 e_{00 ; 0} t_{00} b_{j} y_{i}+18 e_{i 0 ; 0} t_{00} \beta y_{j}+18 e_{j 0 ; 0} t_{00} \beta y_{i} \\
& -9 t_{00} \beta^{3}{ }^{t i y_{j}}-9 t_{00} \beta^{3} t j y_{i}-28 a_{i j} e_{00}^{2} \mathcal{L}_{\hat{X}} \beta \beta \\
& +4 a_{i j} e_{00 ; 0} \mathcal{L}_{\hat{X}} \beta \beta^{2}-18 a_{i j} t_{00} \beta^{2} q_{00}+96 e_{00}^{2} \mathcal{L}_{\hat{X}} \beta b_{i} y_{j} \\
& +96 e_{00}^{2} \mathcal{L}_{\hat{X}} \beta b_{j} y_{i}+54 e_{00}^{2} t_{00} b_{i} b_{j}+9 e_{00} e_{i j} t_{00} \beta^{2} \\
& +18 e_{i 0} e_{j 0} t_{00} \beta^{2}+12 e_{i 0 ; 0} \mathcal{L}_{\hat{X}} \beta \beta^{2} y_{j}+9 e_{i 0 ; 0} t_{00} \beta^{2} b_{j} \\
& +12 e_{j 0 ; 0} \mathcal{L}_{\hat{X}} \beta \beta^{2} y_{i}+9 e_{j 0 ; 0} t_{00} \beta^{2} b_{i}-9 e_{j 0} t_{00} \beta^{3} s_{i} \\
& A_{5}=4 a_{i j} \mathcal{L}_{\hat{X}} t_{0} \beta^{4}-10 e_{i j ; 0} \mathcal{L}_{\hat{X}} \beta \beta^{3}+5 t_{00} \beta^{3} q_{i j} \\
& 5 t_{00} \beta^{3} q_{j i}-28 a_{i j} e_{00}^{2} \mathcal{L}_{\hat{X}} \beta-21 e_{i j ; 0} t_{00} \beta^{2} \\
& +8 e_{i 0 ; 0} t_{00} y_{j}+8 e_{j 0 ; 0} t_{00} y_{i}-2 e_{i 0} \beta^{4} \eta_{j}+4 e_{i j} \mathcal{L}_{\hat{X}} s_{0} \beta^{4} \\
& -4 e_{i j} \beta^{4} \text { eta }+2 e_{j 0} \mathcal{L}_{\hat{X}} s_{i} \beta^{4}-2 e_{j 0} \beta^{4} \eta_{i} \\
& +4 \mathcal{L}_{\hat{X}} a_{i j} \beta^{4} t_{0}+4 \mathcal{L}_{\hat{X}} a_{i j} \beta^{3} s_{0}^{2}+2 \mathcal{L}_{\hat{X}} e_{i 0} \beta^{4} s_{j} \\
& +4 \mathcal{L}_{\hat{X}} e_{i j} \beta^{4} s_{0}+2 \mathcal{L}_{\hat{X}} e_{j 0} \beta^{4} s_{i}-2 e_{00} \mathcal{L}_{\hat{X}} e_{i j} \beta^{3} \\
& -4 e_{i 0} \mathcal{L}_{\hat{X}} e_{j 0} \beta^{3}+2 \mathcal{L}_{\hat{X}} t_{j} \beta^{4} y_{i}+2 \mathcal{L}_{\hat{X}} t_{j} \beta^{4} y_{j} \\
& +2 e_{i 0} \mathcal{L}_{\hat{X}} s_{j} \beta^{4}-2 e_{i j} \mathcal{L}_{\hat{X}} e_{00} \beta^{3}-4 e_{j 0} \mathcal{L}_{\hat{X}} e_{i 0} \beta^{3} \\
& -2 e_{j 0 ; 0} \mathcal{L}_{\hat{X}} b_{i} \beta^{3}+4 \mathcal{L}_{\hat{X}} a_{i j} \beta^{3} q_{00}-2 \mathcal{L}_{\hat{X}} e_{i 0 ; 0} \beta^{3} b_{j} \\
& -2 \mathcal{L}_{\hat{X}} e_{j 0 ; 0} \beta^{3} b_{i}+4 \mathcal{L}_{\hat{X}} q_{0 i} \beta^{3} y_{j}+4 \mathcal{L}_{\hat{X}} q_{0 j} \beta^{3} y_{i} \\
& +2 \mathcal{L}_{\hat{X}} y_{i} \beta^{4} t_{j}+2 \mathcal{L}_{\hat{X}} y_{j} \beta^{4} t_{i}+4 a_{i j} \mathcal{L}_{\hat{X}} q_{00} \beta^{3} \\
& -6 e_{i 0 ; 0} \mathcal{L}_{\hat{X}} y_{j} \beta^{2}-6 e_{j 0 ; 0} \mathcal{L}_{\hat{X}} y_{i} \beta^{2}-6\left(\mathcal{L}_{\hat{X}} e_{i 0}\right)_{; 0} \beta^{2} y_{j} \\
& -6\left(\mathcal{L}_{\hat{X}} e_{j 0}\right)_{; 0} \beta^{2} y_{i}+3 e_{00 ; 0} \mathcal{L}_{\hat{X}} y_{i} y_{j}+3 e_{00 ; 0} \mathcal{L}_{\hat{X}} y_{j} y_{i} \\
& +3 \mathcal{L}_{\hat{X}} e_{00 ; 0} y_{i} y_{j}+4 \mathcal{L}_{\hat{X}} y_{i} \beta^{3} q_{0 j}+4 \mathcal{L}_{\hat{X}} y_{j} \beta^{3} q_{0 i} \\
& -3 a_{i j} \mathcal{L}_{\hat{X}} e_{00 ; 0} \beta^{2}+8 e_{00}^{2} \mathcal{L}_{\hat{X}} a_{i j} \beta-12 e_{00}^{2} \mathcal{L}_{\hat{X}} b_{i} y_{j} \\
& -12 e_{00}^{2} \mathcal{L}_{\hat{X}} b_{j} y_{i}-12 e_{00}^{2} \mathcal{L}_{\hat{X}} y_{i} b_{j}-12 e_{00}^{2} \mathcal{L}_{\hat{X}} y_{j} b_{i} \\
& -3 e_{00 ; 0} \mathcal{L}_{\hat{X}} a_{i j} \beta^{2}-2 e_{i 0 ; 0} \mathcal{L}_{\hat{X}} b_{j} \beta^{3}-14 e_{00 ; 0} \mathcal{L}_{\hat{X}} \beta \beta b_{i} b_{j} \\
& +72 e_{i 0} \mathcal{L}_{\hat{X}} \beta \beta s_{0} y_{j}+45 e_{i 0} t_{00} \beta b_{j} s_{0}
\end{aligned}
$$




$$
\begin{aligned}
& +72 e_{j 0} \mathcal{L}_{\hat{X}} \beta \beta s_{0} y_{i}+45 e_{j 0} t_{00} \beta b_{i} s_{0}+32 \mathcal{L}_{\hat{X}} \beta \beta b_{i} q_{00} y_{j} \\
& +32 \mathcal{L}_{\hat{X}} \beta \beta b_{j} q_{00} y_{i}+40 \mathcal{L}_{\hat{X}} \beta \beta t_{0} y_{i} y_{j} \\
& +28 t_{00} \beta b_{i} b_{j} q_{00}+3 t_{00} \beta b_{i} t_{0} y_{j}+3 t_{00} \beta b_{j} t_{0} y_{i} \\
& -42 t_{00} \beta s_{0} s_{i} y_{j}-42 t_{00} \beta s_{0} s_{j} y_{i}+16 e_{00} \mathcal{L}_{\hat{X}} b_{i} \beta^{2} b_{j} s_{0} \\
& +16 e_{00} \mathcal{L}_{\hat{X}} b_{j} \beta^{2} b_{i} s_{0}+16 e_{00} \mathcal{L}_{\hat{X}} s_{0} \beta^{2} b_{i} b_{j}-16 e_{00} \beta^{2} b_{i} b_{j} \eta \\
& +16 t_{00} \beta^{2} b_{i} s_{j} t_{0}+16 t_{00} \beta^{2} b_{j} s_{i} t_{0} \\
& -24 e_{00} \mathcal{L}_{\hat{X}} e_{00} \beta b_{i} b_{j}+32 e_{00} \mathcal{L}_{\hat{X}} b_{i} \beta s_{0} y_{j}+16 \mathcal{L}_{\hat{X}} e_{00} \beta^{2} b_{i} b_{j} s_{0} \\
& -14 \mathcal{L}_{\hat{X}} \beta \beta^{2} s_{0} s_{i} y_{j}-14 \mathcal{L}_{\hat{X}} \beta \beta^{2} s_{0} s_{j} y_{i} \\
& +8 \mathcal{L}_{\hat{X}} \beta \beta^{2} s_{i} t_{0} y_{j}+8 \mathcal{L}_{\hat{X}} \beta \beta^{2} s_{j} t_{0} y_{i}-4 \mathcal{L}_{\hat{X}} s_{0} \beta^{2} b_{i} s_{0} y_{j} \\
& -4 \mathcal{L}_{\hat{X}} s_{0} \beta^{2} b_{j} s_{0} y_{i}+32 \mathcal{L}_{\hat{X}} e_{00} \beta b_{i} s_{0} y_{j} \\
& +32 \mathcal{L}_{\hat{X}} e_{00} \beta b_{j} s_{0} y_{i}-28 t_{00} \beta^{2} b_{i} b_{j} t_{0}-32 \mathcal{L}_{\hat{X}} s_{0} \beta s_{0} y_{i} y_{j} \\
& +14 t_{00} \beta s_{i} t_{0} y_{j}+14 t_{00} \beta s_{j} t_{0} y_{i} \\
& +32 e_{00} \mathcal{L}_{\hat{X}} b_{j} \beta s_{0} y_{i}+32 e_{00} \mathcal{L}_{\hat{X}} s_{0} \beta b_{i} y_{j}+32 e_{00} \mathcal{L}_{\hat{X}} s_{0} \beta b_{j} y_{i} \\
& +32 e_{00} \mathcal{L}_{\hat{X}} y_{i} \beta b_{j} s_{0}+32 e_{00} \mathcal{L}_{\hat{X}} y_{j} \beta b_{i} s_{0} \\
& -32 e_{00} \beta b_{i} \eta y_{j}-32 e_{00} \beta b_{j} \eta y_{i}+36 e_{i 0} \mathcal{L}_{\hat{X}} \beta \beta^{2} b_{j} s_{0} \\
& +36 e_{j 0} \mathcal{L}_{\hat{X}} \beta \beta^{2} b_{i} s_{0}+28 \mathcal{L}_{\hat{X}} \beta \beta^{2} b_{i} t_{0} y_{j} \\
& +28 \mathcal{L}_{\hat{X}} \beta \beta^{2} b_{j} t_{0} y_{i}+32 \mathcal{L}_{\hat{X}} \beta \beta b_{i} s_{0}^{2} y_{j}+32 \mathcal{L}_{\hat{X}} \beta \beta b_{j} s_{0}^{2} y_{i} \\
& -34 t_{00} \beta^{2} b_{i} s_{0} s_{j}-34 t_{00} \beta^{2} b_{j} s_{0} s_{i} \\
& +28 t_{00} \beta b_{i} b_{j} s_{0}^{2}+96 a_{i j} e_{00} \mathcal{L}_{\hat{X}} \beta \beta s_{0}-56 e_{00} e_{i 0} \mathcal{L}_{\hat{X}} \beta \beta b_{j} \\
& -56 e_{00} e_{j 0} \mathcal{L}_{\hat{X}} \beta \beta b_{i}+48 e_{00} \mathcal{L}_{\hat{X}} \beta \beta s_{i} y_{j} \\
& +48 e_{00} \mathcal{L}_{\hat{X}} \beta \beta s_{j} y_{i}-112 e_{00} \mathcal{L}_{\hat{X}} \beta b_{i} s_{0} y_{j}-112 e_{00} \mathcal{L}_{\hat{X}} \beta b_{j} s_{0} y_{i} \\
& +30 e_{00} t_{00} \beta b_{i} s_{j}+30 e_{00} t_{00} \beta b_{j} s_{i} \\
& -64 e_{00} t_{00} b_{i} b_{j} s_{0}+24 e_{00} \mathcal{L}_{\hat{X}} \beta \beta^{2} b_{i} s_{j}+24 e_{00} \mathcal{L}_{\hat{X}} \beta \beta^{2} b_{j} s_{i} \\
& -4 e_{00} \mathcal{L}_{\hat{X}} b_{j} \beta^{3} s_{i}-4 e_{00} \mathcal{L}_{\hat{X}} s_{i} \beta^{3} b_{j} \\
& -4 e_{00} \mathcal{L}_{\hat{X}} s_{j} \beta^{3} b_{i}+4 e_{00} \beta^{3} b_{i} \eta_{j}+4 e_{00} \beta^{3} b_{j} \eta_{i}-t_{00} \beta^{3} b_{i} t_{j} \\
& -t_{00} \beta^{3} b_{j} t_{i}+8 t_{00} \beta q_{j 0} y_{i}+24 t_{00} b_{i} q_{00} y_{j} \\
& +24 t_{00} b_{j} q_{00} y_{i}-6 e_{i 0} \mathcal{L}_{\hat{X}} b_{j} \beta^{3} s_{0}-6 e_{i 0} \mathcal{L}_{\hat{X}} s_{0} \beta^{3} b_{j} \\
& +6 e_{i 0} \beta^{3} b_{j} \eta-6 e_{j 0} \mathcal{L}_{\hat{X}} b_{i} \beta^{3} s_{0}-6 e_{j 0} \mathcal{L}_{\hat{X}} s_{0} \beta^{3} b_{i} \\
& +6 e_{j 0} \beta^{3} b_{i} e t a-4 \mathcal{L}_{\hat{X}} e_{00} \beta^{3} b_{i} s_{j}-4 \mathcal{L}_{\hat{X}} e_{00} \beta^{3} b_{j} s_{i} \\
& -6 \mathcal{L}_{\hat{X}} e_{i 0} \beta^{3} b_{j} s_{0}-6 \mathcal{L}_{\hat{X}} e_{j 0} \beta^{3} b_{i} s_{0}+8 a_{i j} \mathcal{L}_{\hat{X}} s_{0} \beta^{3} s_{0} \\
& -4 e_{00} \mathcal{L}_{\hat{X}} b_{i} \beta^{3} s_{j}+4 \mathcal{L}_{\hat{X}} t_{0} \beta^{3} s_{i} y_{j}+4 \mathcal{L}_{\hat{X}} t_{0} \beta^{3} s_{j} y_{i} \\
& +4 \mathcal{L}_{\hat{X}} y_{i} \beta^{3} s_{j} t_{0}-2 \mathcal{L}_{\hat{X}} y_{i} \beta^{2} b_{j} s_{0}^{2}-2 \mathcal{L}_{\hat{X}} b_{i} \beta^{2} s_{0}^{2} y_{j} \\
& -2 \mathcal{L}_{\hat{X}} b_{j} \beta^{2} s_{0}^{2} y_{i}-12 e_{00} \mathcal{L}_{\hat{X}} y_{i} \beta^{2} s_{j}-12 e_{00} \mathcal{L}_{\hat{X}} y_{j} \beta^{2} s_{i} \\
& +12 e_{00} \beta^{2} \eta_{i} y_{j}+12 e_{00} \beta^{2} \eta_{j} y_{i}+2 e_{00 ; 0} \mathcal{L}_{\hat{X}} b_{i} \beta^{2} b_{j} \\
& +2 e_{00 ; 0} \mathcal{L}_{\hat{X}} b_{j} \beta^{2} b_{i}+4 \mathcal{L}_{\hat{X}} y_{j} \beta^{3} s_{i} t_{0}-2 \mathcal{L}_{\hat{X}} y_{j} \beta^{2} b_{i} s_{0}^{2} \\
& -12 a_{i j} e_{00} \mathcal{L}_{\hat{X}} s_{0} \beta^{2}+24 a_{i j} e_{00} \beta^{2} \eta
\end{aligned}
$$




$$
\begin{aligned}
& -24 a_{i j} \mathcal{L}_{\hat{X}} e_{00} \beta^{2} s_{0}-12 e_{00}^{2} \mathcal{L}_{\hat{X}} b_{i} \beta b_{j}-12 e_{00}^{2} \mathcal{L}_{\hat{X}} b_{j} \beta b_{i} \\
& +8 e_{00} e_{i 0} \mathcal{L}_{\hat{X}} b_{j} \beta^{2}+4 \mathcal{L}_{\hat{X}} s_{i} \beta^{3} t_{0} y_{j} \\
& +4 \mathcal{L}_{\hat{X}} s_{j} \beta^{3} t_{0} y_{i}-2 \mathcal{L}_{\hat{X}} q_{00} \beta^{2} b_{i} y_{j}-2 \mathcal{L}_{\hat{X}} q_{00} \beta^{2} b_{j} y_{i} \\
& -16 \mathcal{L}_{\hat{X}} t_{0} \beta^{2} y_{i} y_{j}+8 e_{i 0} \mathcal{L}_{\hat{X}} e_{00} \beta^{2} b_{j}-18 e_{i 0} \mathcal{L}_{\hat{X}} s_{0} \beta^{2} y_{j} \\
& -18 e_{i 0} \mathcal{L}_{\hat{X}} y_{j} \beta^{2} s_{0}+18 e_{i 0} \beta^{2} \eta y_{j}+8 e_{j 0} \mathcal{L}_{\hat{X}} e_{00} \beta^{2} b_{i} \\
& -18 e_{j 0} \mathcal{L}_{\hat{X}} s_{0} \beta^{2} y_{i}-18 e_{j 0} \mathcal{L}_{\hat{X}} y_{i} \beta^{2} s_{0}+18 e_{j 0} \beta^{2} \eta y_{i} \\
& +8 e_{00} e_{j 0} \mathcal{L}_{\hat{X}} b_{i} \beta^{2}-24 e_{00} \mathcal{L}_{\hat{X}} a_{i j} \beta^{2} s_{0}+8 e_{00} \mathcal{L}_{\hat{X}} e_{i 0} \beta^{2} b_{j} \\
& +8 e_{00} \mathcal{L}_{\hat{X}} e_{j 0} \beta^{2} b_{i}-12 e_{00} \mathcal{L}_{\hat{X}} s_{i} \beta^{2} y_{j} \\
& -12 e_{00} \mathcal{L}_{\hat{X}} s_{j} \beta^{2} y_{i}+16 e_{00} \mathcal{L}_{\hat{X}} e_{j 0} \beta y_{i}+20 e_{00} \mathcal{L}_{\hat{X}} s_{0} y_{i} y_{j} \\
& +24 e_{00} \mathcal{L}_{\hat{X}} y_{i} s_{0} y_{j}+24 e_{00} \mathcal{L}_{\hat{X}} y_{j} s_{0} y_{i} \\
& -24 e_{00} \eta y_{i} y_{j}+4 e_{00 ; 0} \mathcal{L}_{\hat{X}} b_{i} \beta y_{j}+4 e_{00 ; 0} \mathcal{L}_{\hat{X}} b_{j} \beta y_{i} \\
& +4 e_{00 ; 0} \mathcal{L}_{\hat{X}} y_{i} \beta b_{j}+4 e_{00 ; 0} \mathcal{L}_{\hat{X}} y_{j} \beta b_{i}-2 \mathcal{L}_{\hat{X}} y_{i} \beta^{2} b_{j} q_{00} \\
& -16 \mathcal{L}_{\hat{X}} y_{i} \beta^{2} t_{0} y_{j}-16 \mathcal{L}_{\hat{X}} y_{i} \beta s_{0}^{2} y_{j}-2 \mathcal{L}_{\hat{X}} y_{j} \beta^{2} b_{i} q_{00} \\
& -16 \mathcal{L}_{\hat{X}} y_{j} \beta^{2} t_{0} y_{i}-16 \mathcal{L}_{\hat{X}} y_{j} \beta s_{0}^{2} y_{i}+16 a_{i j} e_{00} \mathcal{L}_{\hat{X}} e_{00} \beta \\
& -12 \mathcal{L}_{\hat{X}} e_{00} \beta^{2} s_{i} y_{j}-12 \mathcal{L}_{\hat{X}} e_{00} \beta^{2} s_{j} y_{i}+2 \mathcal{L}_{\hat{X}} e_{00 ; 0} \beta^{2} b_{i} b_{j} \\
& -18 \mathcal{L}_{\hat{X}} e_{i 0} \beta^{2} s_{0} y_{j}-18 \mathcal{L}_{\hat{X}} e_{j 0} \beta^{2} s_{0} y_{i} \\
& -2 \mathcal{L}_{\hat{X}} b_{i} \beta^{2} q_{00} y_{j}-2 \mathcal{L}_{\hat{X}} b_{j} \beta^{2} q_{00} y_{i}+16 e_{i 0} \mathcal{L}_{\hat{X}} e_{00} \beta y_{j} \\
& +16 e_{j 0} \mathcal{L}_{\hat{X}} e_{00} \beta y_{i}+24 \mathcal{L}_{\hat{X}} e_{00} s_{0} y_{i} y_{j}+4\left(\mathcal{L}_{\hat{X}} e_{00}\right)_{; 0} \beta b_{i} y_{j} \\
& +4\left(\mathcal{L}_{\hat{X}} e_{00}\right)_{; 0} \beta b_{j} y_{i}-16 \mathcal{L}_{\hat{X}} q_{00} \beta y_{i} y_{j}-16 \mathcal{L}_{\hat{X}} y_{i} \beta q_{00} y_{j} \\
& -16 \mathcal{L}_{\hat{X}} y_{j} \beta q_{00} y_{i}+16 e_{00} e_{i 0} \mathcal{L}_{\hat{X}} y_{j} \beta \\
& +16 e_{00} e_{j 0} \mathcal{L}_{\hat{X}} y_{i} \beta-24 e_{00} \mathcal{L}_{\hat{X}} e_{00} b_{i} y_{j}-24 e_{00} \mathcal{L}_{\hat{X}} e_{00} b_{j} y_{i} \\
& +16 e_{00} \mathcal{L}_{\hat{X}} e_{i 0} \beta y_{j}+\left(5 / 2 a_{i j}\right) e_{00 ; 0} t_{00} \\
& +2 \mathcal{L}_{\hat{X}} e_{i j ; 0} \beta^{4}-10 \mathcal{L}_{\hat{X}} t_{0} \beta^{3} b_{i} y_{j}-10 \mathcal{L}_{\hat{X}} t_{0} \beta^{3} b_{j} y_{i} \\
& -10 \mathcal{L}_{\hat{X}} y_{i} \beta^{3} b_{j} t_{0}-2 \mathcal{L}_{\hat{X}} y_{i} \beta^{3} s_{0} s_{j}-10 \mathcal{L}_{\hat{X}} y_{j} \beta^{3} b_{i} t_{0} \\
& -2 \mathcal{L}_{\hat{X}} y_{j} \beta^{3} s_{0} s_{i}-10 \mathcal{L}_{\hat{X}} b_{i} \beta^{3} t_{0} y_{j}-10 \mathcal{L}_{\hat{X}} b_{j} \beta^{3} t_{0} y_{i} \\
& -6 \mathcal{L}_{\hat{X}} s_{0} \beta^{3} s_{i} y_{j}-6 \mathcal{L}_{\hat{X}} s_{0} \beta^{3} s_{j} y_{i}-2 \mathcal{L}_{\hat{X}} s_{i} \beta^{3} s_{0} y_{j} \\
& -2 \mathcal{L}_{\hat{X}} s_{j} \beta^{3} s_{0} y_{i}-6 \mathcal{L}_{\hat{X}} q_{i 0} \beta^{3} y_{j}-6 \mathcal{L}_{\hat{X}} q_{j 0} \beta^{3} y_{i} \\
& -6 \mathcal{L}_{\hat{X}} y_{i} \beta^{3} q_{j 0}-6 \mathcal{L}_{\hat{X}} y_{j} \beta^{3} q_{i 0}-112 e_{00} \mathcal{L}_{\hat{X}} \beta \beta b_{i} b_{j} s_{0} \\
& -21 e_{i 0} t_{00} \beta^{2} s_{j}+12 e_{i 0 ; 0} \mathcal{L}_{\hat{X}} \beta \beta^{2} b_{j}-42 e_{i j} t_{00} \beta^{2} s_{0} \\
& -20 a_{i j} \mathcal{L}_{\hat{X}} \beta \beta^{3} t_{0}-24 a_{i j} \mathcal{L}_{\hat{X}} \beta \beta^{2} s_{0}^{2}-10 e_{i 0} \mathcal{L}_{\hat{X}} \beta \beta^{3} s_{j} \\
& -20 e_{i j} \mathcal{L}_{\hat{X}} \beta \beta^{3} s_{0}-10 e_{j 0} \mathcal{L}_{\hat{X}} \beta \beta^{3} s_{i}-10 \mathcal{L}_{\hat{X}} \beta \beta^{3} t_{i} y_{j} \\
& -10 \mathcal{L}_{\hat{X}} \beta \beta^{3} t_{j} y_{i}-28 \mathcal{L}_{\hat{X}} \beta \beta^{2} q_{0 i} y_{j}-24 \mathcal{L}_{\hat{X}} \beta \beta^{2} q_{0 j} y_{i} \\
& +18 \mathcal{L}_{\hat{X}} \beta \beta^{2} q_{i 0} y_{j}+18 \mathcal{L}_{\hat{X}} \beta \beta^{2} q_{j 0} y_{i}+56 \mathcal{L}_{\hat{X}} \beta s_{0}^{2} y_{i} y_{j} \\
& -12 t_{00} \beta^{2} b_{i} q_{0 j}-6 t_{00} \beta^{2} b_{i} q_{j 0}-20 t_{00} \beta^{2} b_{j} q_{0 i} \\
& -6 t_{00} \beta^{2} b_{j} q_{i 0}-15 t_{00} \beta^{2} t_{i} y_{j}-15 t_{00} \beta^{2} t_{j} y_{i}+24 t_{00} b_{i} s_{0}^{2} y_{j} \\
& +24 t_{00} b_{j} s_{0}^{2} y_{i}+10 t_{00} \beta^{3} s_{i} s_{j}
\end{aligned}
$$




$$
\begin{aligned}
& -30 a_{i j} t_{00} \beta^{2} t_{0}-30 a_{i j} t_{00} \beta s_{0}^{2}+96 e_{00}^{2} \mathcal{L}_{\hat{X}} \beta b_{i} b_{j} \\
& +12 e_{00} e_{i j} \mathcal{L}_{\hat{X}} \beta \beta^{2}+24 e_{i 0} e_{j 0} \mathcal{L}_{\hat{X}} \beta \beta^{2}+32 a_{i j} e_{00} t_{00} s_{0} \\
& +8 a_{i j} e_{00 ; 0} \mathcal{L}_{\hat{X}} \beta \beta-30 a_{i j} t_{00} \beta q_{00}-56 e_{00} e_{i 0} \mathcal{L}_{\hat{X}} \beta y_{j} \\
& -32 e_{00} e_{i 0} t_{00} b_{j}+15 e_{00} e_{i j} t_{00} \beta-56 e_{00} e_{j 0} \mathcal{L}_{\hat{X}} \beta y_{i} \\
& -32 e_{00} e_{j 0} t_{00} b_{i}+16 e_{00} t_{00} s_{i} y_{j}+16 e_{00} t_{00} s_{j} y_{i}-14 e_{00 ; 0} \mathcal{L}_{\hat{X}} \beta b_{i} y_{j} \\
& -14 e_{00 ; 0} \mathcal{L}_{\hat{X}} \beta b_{j} y_{i}-8 e_{00 ; 0} t_{00} b_{i} b_{j} \\
& +30 e_{i 0} e_{j 0} t_{00} \beta+24 e_{i 0} t_{00} s_{0} y_{j}+24 e_{i 0 ; 0} \mathcal{L}_{\hat{X}} \beta \beta y_{j} \\
& +15 e_{i 0 ; 0} t_{00} \beta b_{j}+24 e_{j 0} t_{00} s_{0} y_{i}+24 e_{j 0 ; 0} \mathcal{L}_{\hat{X}} \beta \beta y_{i} \\
& +15 e_{j 0 ; 0} t_{00} \beta b_{i}+56 \mathcal{L}_{\hat{X}} \beta q_{00} y_{i} y_{j}-40 t_{00} \beta q_{0 i} y_{j} \\
& -30 t_{00} \beta q_{0 j} y_{i}+8 t_{00} \beta q_{i 0} y_{j}-21 e_{j 0} t_{00} \beta^{2} s_{i} \\
& +12 e_{j 0 ; 0} \mathcal{L}_{\hat{X}} \beta \beta^{2} b_{i}-24 a_{i j} \mathcal{L}_{\hat{X}} \beta \beta^{2} q_{00} \\
& A_{6}=10 \mathcal{L}_{\hat{X}} \beta \beta^{3} q_{i j}+10 \mathcal{L}_{\hat{X}} \beta \beta^{3} q_{j i}-14 a_{i j} t_{00} s_{0}^{2} \\
& -2 \mathcal{L}_{\hat{X}} t_{i} \beta^{4} b_{j}+2 \mathcal{L}_{\hat{X}} t_{j} \beta^{4} b_{j}+12 a_{i j} \mathcal{L}_{\hat{X}} t_{0} \beta^{3} \\
& -30 e_{i j ; 0} \mathcal{L}_{\hat{X}} \beta \beta^{2}+15 t_{00} \beta^{2} q_{i j}+15 t_{00} \beta^{2} q_{j i} \\
& +4 a_{i j} e_{00 ; 0} \mathcal{L}_{\hat{X}} \beta-14 a_{i j} t_{00} q_{00}+7 e_{00} e_{i j} t_{00}+14 e_{i 0} e_{j 0} t_{00} \\
& +12 e_{i 0 ; 0} \mathcal{L}_{\hat{X}} \beta y_{j}+7 e_{i 0 ; 0} t_{00} b_{j}-19 e_{i j ; 0} t_{00} \beta \\
& +12 e_{j 0 ; 0} \mathcal{L}_{\hat{X}} \beta y_{i}+7 e_{j 0 ; 0} t_{00} b_{i}-18 t_{00} q_{0 i} y_{j}-14 t_{00} q_{0 j} y_{i} \\
& +12 a_{i j} \beta^{3} t_{0}+12 a_{i j} \beta^{2} s_{0}^{2}-4 \mathcal{L}_{\hat{X}} s_{i} \beta^{4} s_{j}-4 \mathcal{L}_{\hat{X}} s_{j} \beta^{4} s_{i} \\
& -6 e_{00} \mathcal{L}_{\hat{X}} e_{i j} \beta^{2}-12 e_{i 0} \mathcal{L}_{\hat{X}} e_{j 0} \beta^{2}-6 e_{i 0 ; 0} \mathcal{L}_{\hat{X}} b_{j} \beta^{2} \\
& +4 \mathcal{L}_{\hat{X}} b_{i} \beta^{3} q_{0 j}+2 \mathcal{L}_{\hat{X}} b_{i} \beta^{3} q_{j 0}+4 \mathcal{L}_{\hat{X}} b_{j} \beta^{3} q_{0 i} \\
& +2 \mathcal{L}_{\hat{X}} b_{j} \beta^{3} q_{i 0}+4 \mathcal{L}_{\hat{X}} q_{0 i} \beta^{3} b_{j}+4 \mathcal{L}_{\hat{X}} q_{0 j} \beta^{3} b_{i}+2 \mathcal{L}_{\hat{X}} q_{i 0} \beta^{3} b_{j} \\
& +2 \mathcal{L}_{\hat{X}} q_{j 0} \beta^{3} b_{i}+6 \mathcal{L}_{\hat{X}} t_{j} \beta^{3} y_{i}+6 \mathcal{L}_{\hat{X}} t_{j} \beta^{3} y_{j} \\
& -6 e_{i j} \mathcal{L}_{\hat{X}} e_{00} \beta^{2}-12 e_{j 0} \mathcal{L}_{\hat{X}} e_{i 0} \beta^{2}-6 e_{j 0 ; 0} \mathcal{L}_{\hat{X}} b_{i} \beta^{2} \\
& +12 a_{i j} \beta^{2} q_{00}-6 \mathcal{L}_{\hat{X}} e_{i 0 ; 0} \beta^{2} b_{j}-6 \mathcal{L}_{\hat{X}} e_{j 0 ; 0} \beta^{2} b_{i} \\
& +12 \mathcal{L}_{\hat{X}} q_{0 i} \beta^{2} y_{j}+12 \mathcal{L}_{\hat{X}} q_{0 j} \beta^{2} y_{i}-6 \mathcal{L}_{\hat{X}} q_{i 0} \beta^{2} y_{j} \\
& -6 \mathcal{L}_{\hat{X}} q_{j 0} \beta^{2} y_{i}+6 \mathcal{L}_{\hat{X}} y_{i} \beta^{3} t_{j}+6 \mathcal{L}_{\hat{X}} y_{j} \beta^{3} t_{i} \\
& +12 a_{i j} \mathcal{L}_{\hat{X}} q_{00} \beta^{2}-12 e_{00}^{2} \mathcal{L}_{\hat{X}} b_{i} b_{j}-12 e_{00}^{2} \mathcal{L}_{\hat{X}} b_{j} b_{i} \\
& -6 e_{i 0 ; 0} \mathcal{L}_{\hat{X}} y_{j} \beta-6 e_{j 0 ; 0} \mathcal{L}_{\hat{X}} y_{i} \beta+2 \mathcal{L}_{\hat{X}} e_{00 ; 0} b_{i} y_{j} \\
& +2 \mathcal{L}_{\hat{X}} e_{00 ; 0} b_{j} y_{i}-6 \mathcal{L}_{\hat{X}} e_{i 0 ; 0} \beta y_{j}-6 \mathcal{L}_{\hat{X}} e_{j 0 ; 0} \beta y_{i} \\
& -8 \mathcal{L}_{\hat{X}} q_{00} y_{i} y_{j}-8 \mathcal{L}_{\hat{X}} y_{i} q_{00} y_{j}-8 \mathcal{L}_{\hat{X}} y_{j} q_{00} y_{i} \\
& +12 \mathcal{L}_{\hat{X}} y_{i} \beta^{2} q_{0 j}-6 \mathcal{L}_{\hat{X}} y_{i} \beta^{2} q_{j 0}-8 \mathcal{L}_{\hat{X}} y_{i} s_{0}^{2} y_{j} \\
& +12 \mathcal{L}_{\hat{X}} y_{j} \beta^{2} q_{0 i}-6 \mathcal{L}_{\hat{X}} y_{j} \beta^{2} q_{i 0}-8 \mathcal{L}_{\hat{X}} y_{j} s_{0}^{2} y_{i} \\
& +8 a_{i j} e_{00} \mathcal{L}_{\hat{X}} e_{00}-3 a_{i j} \mathcal{L}_{\hat{X}} e_{00 ; 0} \beta+8 e_{00} e_{i 0} \mathcal{L}_{\hat{X}} y_{j} \\
& +8 e_{00} e_{j 0} \mathcal{L}_{\hat{X}} y_{i}+8 e_{00} \mathcal{L}_{\hat{X}} e_{i 0} y_{j}+8 e_{00} \mathcal{L}_{\hat{X}} e_{j 0} y_{i} \\
& -3 e_{00 ; 0} a_{i j} \beta+2 e_{00 ; 0} \mathcal{L}_{\hat{X}} b_{i} y_{j}+2 e_{00 ; 0} \mathcal{L}_{\hat{X}} b_{j} y_{i} \\
& +2 e_{00 ; 0} \mathcal{L}_{\hat{X}} y_{i} b_{j}+2 e_{00 ; 0} \mathcal{L}_{\hat{X}} y_{j} b_{i}+8 e_{i 0} \mathcal{L}_{\hat{X}} e_{00} y_{j}
\end{aligned}
$$




$$
\begin{aligned}
& +8 e_{j 0} \mathcal{L}_{\hat{X}} e_{00} y_{i}+8 \mathcal{L}_{\hat{X}} \beta \beta^{2} b_{j} s_{i} t_{0}-16 \mathcal{L}_{\hat{X}} s_{0} \beta^{2} b_{i} b_{j} s_{0} \\
& +32 e_{00} \mathcal{L}_{\hat{X}} b_{i} \beta b_{j} s_{0}+32 e_{00} \mathcal{L}_{\hat{X}} b_{j} \beta b_{i} s_{0}+32 e_{00} \mathcal{L}_{\hat{X}} s_{0} \beta b_{i} b_{j} \\
& -32 e_{00} \beta b_{i} b_{j} \eta+20 t_{00} \beta b_{i} s_{j} t_{0}+20 t_{00} \beta b_{j} s_{i} t_{0} \\
& +32 \mathcal{L}_{\hat{X}} e_{00} \beta b_{i} b_{j} s_{0}+16 \mathcal{L}_{\hat{X}} \beta \beta s_{i} t_{0} y_{j}+16 \mathcal{L}_{\hat{X}} \beta \beta s_{j} t_{0} y_{i} \\
& -20 \mathcal{L}_{\hat{X}} s_{0} \beta b_{i} s_{0} y_{j}-20 \mathcal{L}_{\hat{X}} s_{0} \beta b_{j} s_{0} y_{i} \\
& -16 \mathcal{L}_{\hat{X}} \beta \beta^{2} b_{i} b_{j} t_{0}+8 \mathcal{L}_{\hat{X}} \beta \beta^{2} b_{i} s_{j} t_{0}+72 e_{j 0} \mathcal{L}_{\hat{X}} \beta \beta b_{i} s_{0} \\
& +56 \mathcal{L}_{\hat{X}} \beta \beta b_{i} b_{j} q_{00}-58 \mathcal{L}_{\hat{X}} \beta \beta s_{0} s_{i} y_{j} \\
& -58 \mathcal{L}_{\hat{X}} \beta \beta s_{0} s_{j} y_{i}-60 t_{00} \beta b_{i} b_{j} t_{0}-56 t_{00} \beta b_{i} s_{0} s_{j} \\
& -56 t_{00} \beta b_{j} s_{0} s_{i}-44 \mathcal{L}_{\hat{X}} \beta \beta^{2} b_{i} s_{0} s_{j} \\
& -44 \mathcal{L}_{\hat{X}} \beta \beta^{2} b_{j} s_{0} s_{i}+56 \mathcal{L}_{\hat{X}} \beta \beta b_{i} b_{j} s_{0}^{2}+48 e_{00} \mathcal{L}_{\hat{X}} \beta \beta b_{i} s_{j} \\
& +48 e_{00} \mathcal{L}_{\hat{X}} \beta \beta b_{j} s_{i}-112 e_{00} \mathcal{L}_{\hat{X}} \beta b_{i} b_{j} s_{0} \\
& +72 e_{i 0} \mathcal{L}_{\hat{X}} \beta \beta b_{j} s_{0}-12 e_{00} \mathcal{L}_{\hat{X}} s_{i} \beta^{2} b_{j}-12 e_{00} \mathcal{L}_{\hat{X}} s_{j} \beta^{2} b_{i} \\
& +12 e_{00} \beta^{2} b_{i} \eta_{j}+12 e_{00} \beta^{2} b_{j} \eta_{i}-18 e_{i 0} \mathcal{L}_{\hat{X}} b_{j} \beta^{2} s_{0} \\
& -18 e_{i 0} \mathcal{L}_{\hat{X}} s_{0} \beta^{2} b_{j}+18 e_{i 0} \beta^{2} b_{j} \eta-18 e_{j 0} \mathcal{L}_{\hat{X}} b_{i} \beta^{2} s_{0} \\
& -18 e_{j 0} \mathcal{L}_{\hat{X}} s_{0} \beta^{2} b_{i}+18 e_{j 0} \beta^{2} b_{i} e t a-12 \mathcal{L}_{\hat{X}} e_{00} \beta^{2} b_{i} s_{j} \\
& -12 \mathcal{L}_{\hat{X}} e_{00} \beta^{2} b_{j} s_{i}-18 \mathcal{L}_{\hat{X}} e_{i 0} \beta^{2} b_{j} s_{0}-18 \mathcal{L}_{\hat{X}} e_{j 0} \beta^{2} b_{i} s_{0} \\
& +6 \mathcal{L}_{\hat{X}} s_{j} \beta^{3} b_{i} s_{0}+4 \mathcal{L}_{\hat{X}} s_{j} \beta^{3} b_{i} t_{0} \\
& -8 \mathcal{L}_{\hat{X}} t_{0} \beta^{3} b_{i} b_{j}+4 \mathcal{L}_{\hat{X}} t_{0} \beta^{3} b_{i} s_{j}+4 \mathcal{L}_{\hat{X}} t_{0} \beta^{3} b_{j} s_{i} \\
& +24 a_{i j} \mathcal{L}_{\hat{X}} s_{0} \beta^{2} s_{0}-12 e_{00} \mathcal{L}_{\hat{X}} b_{i} \beta^{2} s_{j}-12 e_{00} \mathcal{L}_{\hat{X}} b_{j} \beta^{2} s_{i} \\
& -22 \mathcal{L}_{\hat{X}} t_{0} \beta^{2} b_{j} y_{i}+12 \mathcal{L}_{\hat{X}} t_{0} \beta^{2} s_{i} y_{j}+12 \mathcal{L}_{\hat{X}} t_{0} \beta^{2} s_{j} y_{i} \\
& -22 \mathcal{L}_{\hat{X}} y_{i} \beta^{2} b_{j} t_{0}+6 \mathcal{L}_{\hat{X}} y_{i} \beta^{2} s_{0} s_{j}+12 \mathcal{L}_{\hat{X}} y_{i} \beta^{2} s_{j} t_{0} \\
& -10 \mathcal{L}_{\hat{X}} y_{i} \beta b_{j} s_{0}^{2}-22 \mathcal{L}_{\hat{X}} y_{j} \beta^{2} b_{i} t_{0}+6 \mathcal{L}_{\hat{X}} y_{j} \beta^{2} s_{0} s_{i} \\
& +12 \mathcal{L}_{\hat{X}} y_{j} \beta^{2} s_{i} t_{0}-10 \mathcal{L}_{\hat{X}} y_{j} \beta b_{i} s_{0}^{2}-8 \mathcal{L}_{\hat{X}} b_{i} \beta^{2} b_{j} q_{00} \\
& -22 \mathcal{L}_{\hat{X}} b_{i} \beta^{2} t_{0} y_{j}-10 \mathcal{L}_{\hat{X}} b_{i} \beta s_{0}^{2} y_{j}-8 \mathcal{L}_{\hat{X}} b_{j} \beta^{2} b_{i} q_{00} \\
& -22 \mathcal{L}_{\hat{X}} b_{j} \beta^{2} t_{0} y_{i}-10 \mathcal{L}_{\hat{X}} b_{j} \beta s_{0}^{2} y_{i}-8 \mathcal{L}_{\hat{X}} q_{00} \beta^{2} b_{i} b_{j} \\
& -6 \mathcal{L}_{\hat{X}} s_{0} \beta^{2} s_{i} y_{j}-6 \mathcal{L}_{\hat{X}} s_{0} \beta^{2} s_{j} y_{i}-12 e_{00} \mathcal{L}_{\hat{X}} y_{j} \beta s_{i} \\
& +16 e_{00} \mathcal{L}_{\hat{X}} y_{j} b_{i} s_{0}+12 e_{00} \beta \eta_{i} y_{j}+12 e_{00} \beta \eta_{j} y_{i} \\
& -16 e_{00} b_{i} \eta y_{j}-16 e_{00} b_{j} \eta y_{i}+4 e_{00 ; 0} \mathcal{L}_{\hat{X}} b_{i} \beta b_{j} \\
& +4 e_{00 ; 0} \mathcal{L}_{\hat{X}} b_{j} \beta b_{i}-12 a_{i j} e_{00} \mathcal{L}_{\hat{X}} s_{0} \beta+24 a_{i j} e_{00} \beta \eta \\
& -24 a_{i j} \mathcal{L}_{\hat{X}} e_{00} \beta s_{0}+16 e_{00} e_{i 0} \mathcal{L}_{\hat{X}} b_{j} \beta+6 \mathcal{L}_{\hat{X}} s_{i} \beta^{2} s_{0} y_{j} \\
& +12 \mathcal{L}_{\hat{X}} s_{i} \beta^{2} t_{0} y_{j}+6 \mathcal{L}_{\hat{X}} s_{j} \beta^{2} s_{0} y_{i}+12 \mathcal{L}_{\hat{X}} s_{j} \beta^{2} t_{0} y_{i} \\
& -22 \mathcal{L}_{\hat{X}} t_{0} \beta^{2} b_{i} y_{j}-16 \mathcal{L}_{\hat{X}} s_{0} s_{0} y_{i} y_{j}-8 \mathcal{L}_{\hat{X}} t_{0} \beta y_{i} y_{j} \\
& -10 \mathcal{L}_{\hat{X}} y_{i} \beta b_{j} q_{00}-8 \mathcal{L}_{\hat{X}} y_{i} \beta t_{0} y_{j}+16 e_{i 0} \mathcal{L}_{\hat{X}} e_{00} \beta b_{j} \\
& -18 e_{i 0} \mathcal{L}_{\hat{X}} s_{0} \beta y_{j}-18 e_{i 0} \mathcal{L}_{\hat{X}} y_{j} \beta s_{0}+18 e_{i 0} \beta \eta y_{j} \\
& +16 e_{j 0} \mathcal{L}_{\hat{X}} e_{00} \beta b_{i}-18 e_{j 0} \mathcal{L}_{\hat{X}} s_{0} \beta y_{i}-18 e_{j 0} \mathcal{L}_{\hat{X}} y_{i} \beta s_{0} \\
& +18 e_{j 0} \beta \eta y_{i}-12 \mathcal{L}_{\hat{X}} e_{00} \beta s_{i} y_{j}-12 \mathcal{L}_{\hat{X}} e_{00} \beta s_{j} y i
\end{aligned}
$$




$$
\begin{aligned}
& +16 e_{00} e_{j 0} \mathcal{L}_{\hat{X}} b_{i} \beta-24 e_{00} a_{i j} \beta s_{0}-24 e_{00} \mathcal{L}_{\hat{X}} e_{00} b_{i} b_{j} \\
& +16 e_{00} \mathcal{L}_{\hat{X}} e_{i 0} \beta b_{j}+16 e_{00} \mathcal{L}_{\hat{X}} e_{j 0} \beta b_{i}+16 e_{00} \mathcal{L}_{\hat{X}} b_{i} s_{0} y_{j} \\
& +16 e_{00} \mathcal{L}_{\hat{X}} b_{j} s_{0} y_{i}+16 e_{00} \mathcal{L}_{\hat{X}} s_{0} b_{i} y_{j} \\
& +16 e_{00} \mathcal{L}_{\hat{X}} s_{0} b_{j} y_{i}-12 e_{00} \mathcal{L}_{\hat{X}} s_{i} \beta y_{j}-12 e_{00} \mathcal{L}_{\hat{X}} s_{j} \beta y_{i} \\
& -12 e_{00} \mathcal{L}_{\hat{X}} y_{i} \beta s_{j}+16 e_{00} \mathcal{L}_{\hat{X}} y_{i} b_{j} s_{0}-10 \mathcal{L}_{\hat{X}} y_{j} \beta b_{i} q_{00} \\
& -8 \mathcal{L}_{\hat{X}} y_{j} \beta t_{0} y_{i}+6 t_{00} s_{i} t_{0} y_{j}+6 t_{00} s_{j} t_{0} y_{i} \\
& +16 \mathcal{L}_{\hat{X}} e_{00} b_{i} s_{0} y_{j}+16 \mathcal{L}_{\hat{X}} e_{00} b_{j} s_{0} y_{i}+4 \mathcal{L}_{\hat{X}} e_{00 ; 0} \beta b_{i} b_{j} \\
& -18 \mathcal{L}_{\hat{X}} e_{i 0} \beta s_{0} y_{j}-18 \mathcal{L}_{\hat{X}} e_{j 0} \beta s_{0} y_{i}-8 \mathcal{L}_{\hat{X}} \beta t_{0} y_{i} y_{j} \\
& -10 \mathcal{L}_{\hat{X}} b_{i} \beta q_{00} y_{j}-10 \mathcal{L}_{\hat{X}} b_{j} \beta q_{00} y_{i}-10 \mathcal{L}_{\hat{X}} q_{00} \beta b_{i} y_{j} \\
& -10 \mathcal{L}_{\hat{X}} q_{00} \beta b_{j} y_{i}+2 \mathcal{L}_{\hat{X}} s_{0} \beta^{3} b_{i} s_{j}+2 \mathcal{L}_{\hat{X}} s_{0} \beta^{3} b_{j} s_{i} \\
& +6 \mathcal{L}_{\hat{X}} s_{i} \beta^{3} b_{j} s_{0}+4 \mathcal{L}_{\hat{X}} s_{i} \beta^{3} b_{j} t_{0}-8 \mathcal{L}_{\hat{X}} b_{i} \beta^{3} b_{j} t_{0} \\
& +6 \mathcal{L}_{\hat{X}} b_{i} \beta^{3} s_{0} s_{j}+4 \mathcal{L}_{\hat{X}} b_{i} \beta^{3} s_{j} t_{0}-8 \mathcal{L}_{\hat{X}} b_{i} \beta^{2} b_{j} s_{0}^{2} \\
& -8 \mathcal{L}_{\hat{X}} b_{j} \beta^{3} b_{i} t_{0}+6 \mathcal{L}_{\hat{X}} b_{j} \beta^{3} s_{0} s_{i}+4 \mathcal{L}_{\hat{X}} b_{j} \beta^{3} s_{i} t_{0} \\
& -8 \mathcal{L}_{\hat{X}} b_{j} \beta^{2} b_{i} s_{0}^{2}-2 \mathcal{L}_{\hat{X}} q_{i j} \beta^{4}-2 \mathcal{L}_{\hat{X}} q_{j i} \beta^{4} \\
& +4 e_{00}^{2} \mathcal{L}_{\hat{X}} a_{i j}+8 e_{i 0} \mathcal{L}_{\hat{X}} s_{j} \beta^{3}-8 e_{i 0} \beta^{3} \eta_{j}+16 e_{i j} \mathcal{L}_{\hat{X}} s_{0} \beta^{3} \\
& -16 e_{i j} \beta^{3} \eta+8 e_{j 0} \mathcal{L}_{\hat{X}} s_{i} \beta^{3}-8 e_{j 0} \beta^{3} \eta_{i}+8 \mathcal{L}_{\hat{X}} e_{i 0} \beta^{3} s_{j} \\
& +16 \mathcal{L}_{\hat{X}} e_{i j} \beta^{3} s_{0}+8 \mathcal{L}_{\hat{X}} e_{j 0} \beta^{3} s_{i}+8 \mathcal{L}_{\hat{X}} e_{i j ; 0} \beta^{3} \\
& +21 e_{i 0} t_{00} b_{j} s_{0}+24 e_{i 0 ; 0} \mathcal{L}_{\hat{X}} \beta \beta b_{j}-38 e_{i j} t_{00} \beta s_{0} \\
& +36 e_{j 0} \mathcal{L}_{\hat{X}} \beta s_{0} y_{i}-19 e_{j 0} t_{00} \beta s_{i}+21 e_{j 0} t_{00} b_{i} s_{0} \\
& +20 \mathcal{L}_{\hat{X}} \beta \beta^{3} s_{i} s_{j}-36 a_{i j} \mathcal{L}_{\hat{X}} \beta \beta^{2} t_{0}-48 a_{i j} \mathcal{L}_{\hat{X}} \beta \beta s_{0}^{2} \\
& -30 e_{i 0} \mathcal{L}_{\hat{X}} \beta \beta^{2} s_{j}-60 e_{i j} \mathcal{L}_{\hat{X}} \beta \beta^{2} s_{0}-30 e_{j 0} \mathcal{L}_{\hat{X}} \beta \beta^{2} s_{i} \\
& -24 \mathcal{L}_{\hat{X}} \beta \beta^{2} b_{i} q_{0 j}-12 \mathcal{L}_{\hat{X}} \beta \beta^{2} b_{i} q_{j 0}-28 \mathcal{L}_{\hat{X}} \beta \beta^{2} b_{j} q_{0 i} \\
& -12 \mathcal{L}_{\hat{X}} \beta \beta^{2} b_{j} q_{i 0}-18 \mathcal{L}_{\hat{X}} \beta \beta^{2} t_{i} y_{j}-18 \mathcal{L}_{\hat{X}} \beta \beta^{2} t_{j} y_{i} \\
& +44 \mathcal{L}_{\hat{X}} \beta b_{i} s_{0}^{2} y_{j}+44 \mathcal{L}_{\hat{X}} \beta b_{j} s_{0}^{2} y_{i}+44 \mathcal{L}_{\hat{X}} \beta b_{i} q_{00} y_{j} \\
& +44 \mathcal{L}_{\hat{X}} \beta b_{j} q_{00} y_{i}-24 t_{00} \beta b_{i} q_{0 j}-12 t_{00} \beta b_{i} q_{j 0} \\
& -34 t_{00} \beta b_{j} q_{0 i}-12 t_{00} \beta b_{j} q_{i 0}-7 t_{00} \beta t_{i} y_{j}-7 t_{00} \beta t_{j} y_{i} \\
& +28 t_{00} b_{i} b_{j} q_{00}-13 t_{00} b_{i} t_{0} y_{j}-13 t_{00} b_{j} t_{0} y_{i} \\
& -22 t_{00} s_{0} s_{i} y_{j}-22 t_{00} s_{0} s_{j} y_{i}+3 t_{00} \beta^{2} b_{i} t_{j}+3 t_{00} \beta^{2} b_{j} t_{i} \\
& +30 t_{00} \beta^{2} s_{i} s_{j}+28 t_{00} b_{i} b_{j} s_{0}^{2}+48 a_{i j} e_{00} \mathcal{L}_{\hat{X}} \beta s_{0} \\
& \text { - } 48 a_{i j} \mathcal{L}_{\hat{X}} \beta \beta q_{00}-14 a_{i j} t_{00} \beta t_{0}-56 e_{00} e_{i 0} \mathcal{L}_{\hat{X}} \beta b_{j} \\
& +24 e_{00} e_{i j} \mathcal{L}_{\hat{X}} \beta \beta-56 e_{00} e_{j 0} \mathcal{L}_{\hat{X}} \beta b_{i}+24 e_{00} \mathcal{L}_{\hat{X}} \beta s_{i} y_{j} \\
& +24 e_{00} \mathcal{L}_{\hat{X}} \beta s_{j} y_{i}+14 e_{00} t_{00} b_{i} s_{j}+14 e_{00} t_{00} b_{j} s_{i} \\
& -14 e_{00 ; 0} \mathcal{L}_{\hat{X}} \beta b_{i} b_{j}+48 e_{i 0} e_{j 0} \mathcal{L}_{\hat{X}} \beta \beta+36 e_{i 0} \mathcal{L}_{\hat{X}} \beta s_{0} y_{j} \\
& -19 e_{i 0} t_{00} \beta s_{j}+24 e_{j 0 ; 0} \mathcal{L}_{\hat{X}} \beta \beta b_{i}-56 \mathcal{L}_{\hat{X}} \beta \beta q_{0 i} y_{j} \\
& -48 \mathcal{L}_{\hat{X}} \beta \beta q_{0 j} y_{i}+6 \mathcal{L}_{\hat{X}} \beta \beta q_{i 0} y_{j}+6 \mathcal{L}_{\hat{X}} \beta \beta q_{j 0} y_{i} \\
& A_{7}=30 \mathcal{L}_{\hat{X}} \beta \beta^{2} q_{i j}+30 \mathcal{L}_{\hat{X}} \beta \beta^{2} q_{j i}-24 a_{i j} \mathcal{L}_{\hat{X}} \beta q_{00}
\end{aligned}
$$




$$
\begin{aligned}
& +12 e_{00} e_{i j} \mathcal{L}_{\hat{X}} \beta+24 e_{i 0} e_{j 0} \mathcal{L}_{\hat{X}} \beta+6 \mathcal{L}_{\hat{X}} t_{j} \beta^{3} b_{j} \\
& +12 a_{i j} \mathcal{L}_{\hat{X}} t_{0} \beta^{2}+12 e_{i 0} \mathcal{L}_{\hat{X}} s_{j} \beta^{2}-6 e_{i 0} t_{00} s_{j} \\
& +12 e_{i 0 ; 0} \mathcal{L}_{\hat{X}} \beta b_{j}-12 e_{i j} t_{00} s_{0}-30 e_{i j ; 0} \mathcal{L}_{\hat{X}} \beta \beta-6 e_{j 0} t_{00} s_{i} \\
& +12 e_{j 0 ; 0} \mathcal{L}_{\hat{X}} \beta b_{i}-28 \mathcal{L}_{\hat{X}} \beta q_{0 i} y_{j}-24 \mathcal{L}_{\hat{X}} \beta q_{0 j} y_{i} \\
& +15 t_{00} \beta q_{i j}+15 t_{00} \beta q_{j i}-12 t_{00} b_{i} q_{0 j}-6 t_{00} b_{i} q_{j 0} \\
& -16 t_{00} b_{j} q_{0 i}-6 t_{00} b_{j} q_{i 0}-24 a_{i j} \mathcal{L}_{\hat{X}} \beta s_{0}^{2}-12 e_{i 0} \beta^{2} \eta_{j} \\
& +24 e_{i j} \mathcal{L}_{\hat{X}} s_{0} \beta^{2}-24 e_{i j} \beta^{2} \eta+12 e_{j 0} \mathcal{L}_{\hat{X}} s_{i} \beta^{2} \\
& -12 e_{j 0} \beta^{2} \eta_{i}+12 \mathcal{L}_{\hat{X}} a_{i j} \beta^{2} t_{0}+12 \mathcal{L}_{\hat{X}} a_{i j} \beta s_{0}^{2} \\
& +12 \mathcal{L}_{\hat{X}} e_{i 0} \beta^{2} s_{j}+24 \mathcal{L}_{\hat{X}} e_{i j} \beta^{2} s_{0}+12 \mathcal{L}_{\hat{X}} e_{j 0} \beta^{2} s_{i} \\
& +8 e_{00} \mathcal{L}_{\hat{X}} e_{j 0} b_{i}-4 e_{00} \mathcal{L}_{\hat{X}} s_{i} y_{j}-4 e_{00} \mathcal{L}_{\hat{X}} s_{j} y_{i} \\
& -4 e_{00} \mathcal{L}_{\hat{X}} y_{i} s_{j}-4 e_{00} \mathcal{L}_{\hat{X}} y_{j} s_{i}+4 e_{00} \eta_{i} y_{j}+4 e_{00} \eta_{j} y_{i} \\
& +2 e_{00 ; 0} \mathcal{L}_{\hat{X}} b_{i} b_{j}+2 e_{00 ; 0} \mathcal{L}_{\hat{X}} b_{j} b_{i}+8 e_{i 0} \mathcal{L}_{\hat{X}} e_{00} b_{j} \\
& -12 e_{i 0} \mathcal{L}_{\hat{X}} e_{j 0} \beta-6 e_{i 0} \mathcal{L}_{\hat{X}} s_{0} y_{j}-6 e_{i 0} \mathcal{L}_{\hat{X}} y_{j} s_{0} \\
& +6 e_{i 0} \eta y_{j}-6 e_{i 0 ; 0} \mathcal{L}_{\hat{X}} b_{j} \beta+12 \mathcal{L}_{\hat{X}} b_{i} \beta^{2} q_{0 j}+6 \mathcal{L}_{\hat{X}} b_{i} \beta^{2} q_{j 0} \\
& -6 \mathcal{L}_{\hat{X}} b_{i} s_{0}^{2} y_{j}+12 \mathcal{L}_{\hat{X}} b_{j} \beta^{2} q_{0 i}+6 \mathcal{L}_{\hat{X}} b_{j} \beta^{2} q_{i 0} \\
& -6 \mathcal{L}_{\hat{X}} b_{j} s_{0}^{2} y_{i}+12 \mathcal{L}_{\hat{X}} q_{0 i} \beta^{2} b_{j}+12 \mathcal{L}_{\hat{X}} q_{0 j} \beta^{2} b_{i} \\
& +6 \mathcal{L}_{\hat{X}} q_{i 0} \beta^{2} b_{j}+6 \mathcal{L}_{\hat{X}} q_{j 0} \beta^{2} b_{i}+6 \mathcal{L}_{\hat{X}} t_{j} \beta^{2} y_{i}+6 \mathcal{L}_{\hat{X}} t_{j} \beta^{2} y_{j} \\
& +8 e_{j 0} \mathcal{L}_{\hat{X}} e_{00} b_{i}-12 e_{j 0} \mathcal{L}_{\hat{X}} e_{i 0} \beta-6 e_{j 0} \mathcal{L}_{\hat{X}} s_{0} y_{i}-6 e_{j 0} \mathcal{L}_{\hat{X}} y_{i} s_{0} \\
& +6 e_{j 0} \eta y_{i}-6 e_{j 0 ; 0} \mathcal{L}_{\hat{X}} b_{i} \beta+12 \mathcal{L}_{\hat{X}} a_{i j} \beta q_{00} \\
& -4 \mathcal{L}_{\hat{X}} e_{00} s_{i} y_{j}-4 \mathcal{L}_{\hat{X}} e_{00} s_{j} y_{i}+2 \mathcal{L}_{\hat{X}} e_{00 ; 0} b_{i} b_{j} \\
& -6 \mathcal{L}_{\hat{X}} e_{i 0} s_{0} y_{j}-6 \mathcal{L}_{\hat{X}} e_{i 0 ; 0} \beta b_{j}-6 \mathcal{L}_{\hat{X}} e_{j 0} s_{0} y_{i} \\
& -6 \mathcal{L}_{\hat{X}} e_{j 0 ; 0} \beta b_{i}-2 \mathcal{L}_{\hat{X}} \beta q_{i 0} y_{j}-2 \mathcal{L}_{\hat{X}} \beta q_{j 0} y_{i}-6 \mathcal{L}_{\hat{X}} b_{i} q_{00} y_{j} \\
& -6 \mathcal{L}_{\hat{X}} b_{j} q_{00} y_{i}-6 \mathcal{L}_{\hat{X}} q_{00} b_{i} y_{j}-6 \mathcal{L}_{\hat{X}} q_{00} b_{j} y_{i} \\
& +12 \mathcal{L}_{\hat{X}} q_{0 i} \beta y_{j}+12 \mathcal{L}_{\hat{X}} q_{0 j} \beta y_{i}-2 \mathcal{L}_{\hat{X}} q_{i 0} \beta y_{j}-2 \mathcal{L}_{\hat{X}} q_{j 0} \beta y_{i} \\
& +6 \mathcal{L}_{\hat{X}} y_{i} \beta^{2} t_{j}-6 \mathcal{L}_{\hat{X}} y_{i} b_{j} s_{0}^{2}+6 \mathcal{L}_{\hat{X}} y_{j} \beta^{2} t_{i} \\
& -6 \mathcal{L}_{\hat{X}} y_{j} b_{i} s_{0}^{2}-4 a_{i j} e_{00} \mathcal{L}_{\hat{X}} s_{0}+8 a_{i j} e_{00} \eta-8 a_{i j} \mathcal{L}_{\hat{X}} e_{00} s_{0} \\
& +12 a_{i j} \mathcal{L}_{\hat{X}} q_{00} \beta+8 e_{00} e_{i 0} \mathcal{L}_{\hat{X}} b_{j}+8 e_{00} e_{j 0} \mathcal{L}_{\hat{X}} b_{i} \\
& -8 e_{00} \mathcal{L}_{\hat{X}} a_{i j} s_{0}+8 e_{00} \mathcal{L}_{\hat{X}} e_{i 0} b_{j}-6 e_{00} \mathcal{L}_{\hat{X}} e_{i j} \beta \\
& +12 \mathcal{L}_{\hat{X}} y_{i} \beta q_{0 j}-2 \mathcal{L}_{\hat{X}} y_{i} \beta q_{j 0}-6 \mathcal{L}_{\hat{X}} y_{i} b_{j} q_{00}+12 \mathcal{L}_{\hat{X}} y_{j} \beta q_{0 i} \\
& -2 \mathcal{L}_{\hat{X}} y_{j} \beta q_{i 0}-6 \mathcal{L}_{\hat{X}} y_{j} b_{i} q_{00}-6 e_{i j} \mathcal{L}_{\hat{X}} e_{00} \beta \\
& -32 \mathcal{L}_{\hat{X}} s_{0} \beta b_{i} b_{j} s_{0}+16 \mathcal{L}_{\hat{X}} \beta \beta b_{i} s_{j} t_{0}+16 \mathcal{L}_{\hat{X}} \beta \beta b_{j} s_{i} t_{0} \\
& -88 \mathcal{L}_{\hat{X}} \beta \beta b_{i} b_{j} t_{0}-88 \mathcal{L}_{\hat{X}} \beta \beta b_{i} s_{0} s_{j}-88 \mathcal{L}_{\hat{X}} \beta \beta b_{j} s_{0} s_{i} \\
& +16 e_{00} \mathcal{L}_{\hat{X}} s_{0} b_{i} b_{j}-12 e_{00} \mathcal{L}_{\hat{X}} s_{i} \beta b_{j} \\
& -12 e_{00} \mathcal{L}_{\hat{X}} s_{j} \beta b_{i}+12 e_{00} \beta b_{i} \eta_{j}+12 e_{00} \beta b_{j} \eta_{i}-16 e_{00} b_{i} b_{j} \eta \\
& +10 \mathcal{L}_{\hat{X}} s_{i} \beta s_{0} y_{j}-18 e_{i 0} \mathcal{L}_{\hat{X}} b_{j} \beta s_{0} \\
& -18 e_{i 0} \mathcal{L}_{\hat{X}} s_{0} \beta b_{j}+18 e_{i 0} \beta b_{j} \eta-18 e_{j 0} \mathcal{L}_{\hat{X}} b_{i} \beta s_{0}
\end{aligned}
$$




$$
\begin{aligned}
& -18 e_{j 0} \mathcal{L}_{\hat{X}} s_{0} \beta b_{i}+18 e_{j 0} \beta b_{i} \eta-12 \mathcal{L}_{\hat{X}} e_{00} \beta b_{i} s_{j} \\
& -12 \mathcal{L}_{\hat{X}} e_{00} \beta b_{j} s_{i}+16 \mathcal{L}_{\hat{X}} e_{00} b_{i} b_{j} s_{0}-18 \mathcal{L}_{\hat{X}} e_{i 0} \beta b_{j} s_{0} \\
& -18 \mathcal{L}_{\hat{X}} e_{j 0} \beta b_{i} s_{0}+12 \mathcal{L}_{\hat{X}} s_{j} \beta^{2} b_{i} t_{0}-16 \mathcal{L}_{\hat{X}} t_{0} \beta^{2} b_{i} b_{j} \\
& +12 \mathcal{L}_{\hat{X}} t_{0} \beta^{2} b_{i} s_{j}+12 \mathcal{L}_{\hat{X}} t_{0} \beta^{2} b_{j} s_{i}+24 a_{i j} \mathcal{L}_{\hat{X}} s_{0} \beta s_{0} \\
& -12 e_{00} \mathcal{L}_{\hat{X}} b_{i} \beta s_{j}+16 e_{00} \mathcal{L}_{\hat{X}} b_{i} b_{j} s_{0}-12 e_{00} \mathcal{L}_{\hat{X}} b_{j} \beta s_{i} \\
& +16 e_{00} \mathcal{L}_{\hat{X}} b_{j} b_{i} s_{0}-14 \mathcal{L}_{\hat{X}} y_{i} \beta b_{j} t_{0}+10 \mathcal{L}_{\hat{X}} y_{i} \beta s_{0} s_{j} \\
& +12 \mathcal{L}_{\hat{X}} y_{i} \beta s_{j} t_{0}-14 \mathcal{L}_{\hat{X}} y_{j} \beta b_{i} t_{0}+10 \mathcal{L}_{\hat{X}} y_{j} \beta s_{0} s_{i} \\
& +12 \mathcal{L}_{\hat{X}} y_{j} \beta s_{i} t_{0}+8 \mathcal{L}_{\hat{X}} \beta s_{i} t_{0} y_{j}+8 \mathcal{L}_{\hat{X}} \beta s_{j} t_{0} y_{i} \\
& -16 \mathcal{L}_{\hat{X}} b_{i} \beta b_{j} q_{00}-14 \mathcal{L}_{\hat{X}} b_{i} \beta t_{0} y_{j}-16 \mathcal{L}_{\hat{X}} b_{j} \beta b_{i} q_{00} \\
& -14 \mathcal{L}_{\hat{X}} b_{j} \beta t_{0} y_{i}-16 \mathcal{L}_{\hat{X}} q_{00} \beta b_{i} b_{j} \\
& -2 \mathcal{L}_{\hat{X}} s_{0} \beta s_{i} y_{j}-2 \mathcal{L}_{\hat{X}} s_{0} \beta s_{j} y_{i}-12 \mathcal{L}_{\hat{X}} s_{0} b_{i} s_{0} y_{j}-12 \mathcal{L}_{\hat{X}} s_{0} b_{j} s_{0} y_{i} \\
& +8 t_{00} b_{i} s_{j} t_{0}+8 t_{00} b_{j} s_{i} t_{0}+12 \mathcal{L}_{\hat{X}} s_{i} \beta t_{0} y_{j} \\
& +10 \mathcal{L}_{\hat{X}} s_{j} \beta s_{0} y_{i}+12 \mathcal{L}_{\hat{X}} s_{j} \beta t_{0} y_{i}-14 \mathcal{L}_{\hat{X}} t_{0} \beta\left(b_{i} y_{j}+b_{j} y_{i}\right) \\
& +12 \mathcal{L}_{\hat{X}} t_{0} \beta s_{i} y_{j}+12 \mathcal{L}_{\hat{X}} t_{0} \beta s_{j} y_{i}+6 \mathcal{L}_{\hat{X}} s_{0} \beta^{2} b_{i} s_{j} \\
& +6 \mathcal{L}_{\hat{X}} s_{0} \beta^{2} b_{j} s_{i}+18 \mathcal{L}_{\hat{X}} s_{i} \beta^{2} b_{j} s_{0}+12 \mathcal{L}_{\hat{X}} s_{i} \beta^{2} b_{j} t_{0} \\
& +18 \mathcal{L}_{\hat{X}} s_{j} \beta^{2} b_{i} s_{0}-16 \mathcal{L}_{\hat{X}} b_{i} \beta^{2} b_{j} t_{0}+18 \mathcal{L}_{\hat{X}} b_{i} \beta^{2} s_{0} s_{j} \\
& +12 \mathcal{L}_{\hat{X}} b_{i} \beta^{2} s_{j} t_{0}-16 \mathcal{L}_{\hat{X}} b_{i} \beta b_{j} s_{0}^{2}-16 \mathcal{L}_{\hat{X}} b_{j} \beta^{2} b_{i} t_{0} \\
& +18 \mathcal{L}_{\hat{X}} b_{j} \beta^{2} s_{0} s_{i}+12 \mathcal{L}_{\hat{X}} b_{j} \beta^{2} s_{i} t_{0}-16 \mathcal{L}_{\hat{X}} b_{j} \beta b_{i} s_{0}^{2} \\
& -6 e_{i j ; 0} t_{00}+12\left(\mathcal{L}_{\hat{X}} e_{i j}\right)_{; 0} \beta^{2}-a_{i j}\left(\mathcal{L}_{\hat{X}} e_{00}\right)_{; 0} \\
& -e_{00 ; 0} \mathcal{L}_{\hat{X}} a_{i j}-2 e_{i 0 ; 0} \mathcal{L}_{\hat{X}} y_{j}-2 e_{j 0 ; 0} \mathcal{L}_{\hat{X}} y_{i}-2\left(\mathcal{L}_{\hat{X}} e_{i 0}\right)_{; 0} y_{j} \\
& -2\left(\mathcal{L}_{\hat{X}} e_{j 0}\right)_{; 0} y_{i}-2 \mathcal{L}_{\hat{X}} b_{i} \beta^{3} t_{j}-2 \mathcal{L}_{\hat{X}} b_{j} \beta^{3} t_{i} \\
& -16 \mathcal{L}_{\hat{X}} s_{i} \beta^{3} s_{j}-16 \mathcal{L}_{\hat{X}} s_{j} \beta^{3} s_{i}-8 \mathcal{L}_{\hat{X}} t_{i} \beta^{3} b_{j}-2 \mathcal{L}_{\hat{X}} t_{j} \beta^{3} b_{i} \\
& -8 \mathcal{L}_{\hat{X}} q_{i j} \beta^{3}-8 \mathcal{L}_{\hat{X}} q_{j i} \beta^{3}-26 t_{00} b_{i} s_{0} s_{j} \\
& +12 \mathcal{L}_{\hat{X}} \beta \beta^{2} b_{i} t_{j}+12 \mathcal{L}_{\hat{X}} \beta \beta^{2} b_{j} t_{i}+60 \mathcal{L}_{\hat{X}} \beta \beta^{2} s_{i} s_{j} \\
& +56 \mathcal{L}_{\hat{X}} \beta b_{i} b_{j} s_{0}^{2}-12 a_{i j} \mathcal{L}_{\hat{X}} \beta \beta t_{0}+24 e_{00} \mathcal{L}_{\hat{X}} \beta b_{i} s_{j} \\
& +24 e_{00} \mathcal{L}_{\hat{X}} \beta b_{j} s_{i}-30 e_{i 0} \mathcal{L}_{\hat{X}} \beta \beta s_{j}+36 e_{i 0} \mathcal{L}_{\hat{X}} \beta b_{j} s_{0} \\
& -60 e_{i j} \mathcal{L}_{\hat{X}} \beta \beta s_{0}-30 e_{j 0} \mathcal{L}_{\hat{X}} \beta \beta s_{i}+36 e_{j 0} \mathcal{L}_{\hat{X}} \beta b_{i} s_{0} \\
& -48 \mathcal{L}_{\hat{X}} \beta \beta b_{i} q_{0 j}-24 \mathcal{L}_{\hat{X}} \beta \beta b_{i} q_{j 0}-56 \mathcal{L}_{\hat{X}} \beta \beta b_{j} q_{0 i} \\
& -24 \mathcal{L}_{\hat{X}} \beta \beta b_{j} q_{i 0}-6 \mathcal{L}_{\hat{X}} \beta \beta t_{i} y_{j}-6 \mathcal{L}_{\hat{X}} \beta \beta t_{j} y_{i} \\
& +56 \mathcal{L}_{\hat{X}} \beta b_{i} b_{j} q_{00}-28 \mathcal{L}_{\hat{X}} \beta b_{i} t_{0} y_{j}-28 \mathcal{L}_{\hat{X}} \beta b_{j} t_{0} y_{i}-34 \mathcal{L}_{\hat{X}} \beta s_{0} s_{i} y_{j} \\
& -34 \mathcal{L}_{\hat{X}} \beta s_{0} s_{j} y_{i}+9 t_{00} \beta b_{i} t_{j} \\
& +9 t_{00} \beta b_{j} t_{i}+30 t_{00} \beta s_{i} s_{j}-40 t_{00} b_{i} b_{j} t_{0}-26 t_{00} b_{j} s_{0} s_{i} \\
& A_{8}=-20 e_{i j} \mathcal{L}_{\hat{X}} \beta s_{0}-10 e_{j 0} \mathcal{L}_{\hat{X}} \beta s_{i}+30 \mathcal{L}_{\hat{X}} \beta \beta q_{i j} \\
& +30 \mathcal{L}_{\hat{X}} \beta \beta q_{j i}-24 \mathcal{L}_{\hat{X}} \beta b_{i} q_{0 j}-12 \mathcal{L}_{\hat{X}} \beta b_{i} q_{j 0} \\
& -28 \mathcal{L}_{\hat{X}} \beta b_{j} q_{0 i}-12 \mathcal{L}_{\hat{X}} \beta b_{j} q_{i 0}+5 t_{00} b_{i} t_{j}+5 t_{00} b_{j} t_{i} \\
& +10 t_{00} s_{i} s_{j}-12 \mathcal{L}_{\hat{X}} t_{i} \beta^{2} b_{j}-6 \mathcal{L}_{\hat{X}} t_{j} \beta^{2} b_{i}
\end{aligned}
$$




$$
\begin{aligned}
& +6 \mathcal{L}_{\hat{X}} t_{j} \beta^{2} b_{j}+4 a_{i j} \mathcal{L}_{\hat{X}} \beta t_{0}+8 a_{i j} \mathcal{L}_{\hat{X}} s_{0} s_{0}+4 a_{i j} \mathcal{L}_{\hat{X}} t_{0} \beta-4 e_{00 ; 0} \mathcal{L}_{\hat{X}} b_{i} s_{j} \\
& -4 e_{00 ; 0} \mathcal{L}_{\hat{X}} b_{j} s_{i}-4 e_{00 ; 0} \mathcal{L}_{\hat{X}} s_{i} b_{j} \\
& -4 e_{00 ; 0} \mathcal{L}_{\hat{X}} s_{j} b_{i}+4 e_{00 ; 0} b_{i} \eta_{j}+4 e_{00 ; 0} b_{j} \eta_{i}-6 e_{i 0} \mathcal{L}_{\hat{X}} b_{j} s_{0} \\
& -6 e_{i 0} \mathcal{L}_{\hat{X}} s_{0} b_{j}+8 e_{i 0} \mathcal{L}_{\hat{X}} s_{j} \beta-10 e_{i 0} \mathcal{L}_{\hat{X}} \beta s_{j} \\
& -8 e_{i 0} \beta \eta_{j}+6 e_{i 0} b_{j} \eta+16 e_{i j} \mathcal{L}_{\hat{X}} s_{0} \beta-16 e_{i j} \beta \eta \\
& -6 e_{j 0} \mathcal{L}_{\hat{X}} b_{i} s_{0}-6 e_{j 0} \mathcal{L}_{\hat{X}} s_{0} b_{i}+8 e_{j 0} \mathcal{L}_{\hat{X}} s_{i} \beta-8 e_{j 0} \beta \eta_{i} \\
& +6 e_{j 0} b_{i} \eta+4 \mathcal{L}_{\hat{X}} a_{i j} \beta t_{0}-4 \mathcal{L}_{\hat{X}} e_{00 ; 0} b_{i} s_{j} \\
& -4 \mathcal{L}_{\hat{X}} e_{00 ; 0} b_{j} s_{i}+8 \mathcal{L}_{\hat{X}} e_{i 0} \beta s_{j}-6 \mathcal{L}_{\hat{X}} e_{i 0} b_{j} s_{0}+16 \mathcal{L}_{\hat{X}} e_{i j} \beta s_{0} \\
& +8 \mathcal{L}_{\hat{X}} e_{j 0} \beta s_{i}-6 \mathcal{L}_{\hat{X}} e_{j 0} b_{i} s_{0}-6 \mathcal{L}_{\hat{X}} b_{i} \beta^{2} t_{j}-8 \mathcal{L}_{\hat{X}} b_{i} b_{j} s_{0}^{2} \\
& -6 \mathcal{L}_{\hat{X}} b_{j} \beta^{2} t_{i}-8 \mathcal{L}_{\hat{X}} b_{j} b_{i} s_{0}^{2}-24 \mathcal{L}_{\hat{X}} s_{i} \beta^{2} s_{j} \\
& -24 \mathcal{L}_{\hat{X}} s_{j} \beta^{2} s_{i}+2 \mathcal{L}_{\hat{X}} \beta t_{i} y_{j}+2 \mathcal{L}_{\hat{X}} \beta t_{j} y_{i}+12 \mathcal{L}_{\hat{X}} b_{i} \beta q_{0 j} \\
& +6 \mathcal{L}_{\hat{X}} b_{i} \beta q_{j 0}-8 \mathcal{L}_{\hat{X}} b_{i} b_{j} q_{00}-2 \mathcal{L}_{\hat{X}} b_{i} t_{0} y_{j} \\
& +12 \mathcal{L}_{\hat{X}} b_{j} \beta q_{0 i}+6 \mathcal{L}_{\hat{X}} b_{j} \beta q_{i 0}-8 \mathcal{L}_{\hat{X}} b_{j} b_{i} q_{00}-2 \mathcal{L}_{\hat{X}} b_{j} t_{0} y_{i} \\
& -8 \mathcal{L}_{\hat{X}} q_{00} b_{i} b_{j}+12 \mathcal{L}_{\hat{X}} q_{0 i} \beta b_{j}+12 \mathcal{L}_{\hat{X}} q_{0 j} \beta b_{i} \\
& +6 \mathcal{L}_{\hat{X}} q_{i 0} \beta b_{j}+6 \mathcal{L}_{\hat{X}} q_{j 0} \beta b_{i}+4 \mathcal{L}_{\hat{X}} s_{i} s_{0} y_{j}+4 \mathcal{L}_{\hat{X}} s_{i} t_{0} y_{j} \\
& +4 \mathcal{L}_{\hat{X}} s_{j} s_{0} y_{i}+4 \mathcal{L}_{\hat{X}} s_{j} t_{0} y_{i}-2 \mathcal{L}_{\hat{X}} t_{0} b_{i} y_{j} \\
& -2 \mathcal{L}_{\hat{X}} t_{0} b_{j} y_{i}+4 \mathcal{L}_{\hat{X}} t_{0} s_{i} y_{j}+4 \mathcal{L}_{\hat{X}} t_{0} s_{j} y_{i}+2 \mathcal{L}_{\hat{X}} t_{j} \beta y_{i} \\
& +2 \mathcal{L}_{\hat{X}} t_{j} \beta y_{j}+2 \mathcal{L}_{\hat{X}} y_{i} \beta t_{j}-2 \mathcal{L}_{\hat{X}} y_{i} b_{j} t_{0} \\
& +4 \mathcal{L}_{\hat{X}} y_{i} s_{0} s_{j}+4 \mathcal{L}_{\hat{X}} y_{i} s_{j} t_{0}+2 \mathcal{L}_{\hat{X}} y_{j} \beta t_{i}-2 \mathcal{L}_{\hat{X}} y_{j} b_{i} t_{0} \\
& +4 \mathcal{L}_{\hat{X}} y_{j} s_{0} s_{i}+4 \mathcal{L}_{\hat{X}} y_{j} s_{i} t_{0}-8 \mathcal{L}_{\hat{X}} t_{0} \beta b_{i} b_{j} \\
& +12 \mathcal{L}_{\hat{X}} t_{0} \beta b_{i} s_{j}+12 \mathcal{L}_{\hat{X}} t_{0} \beta b_{j} s_{i}+6 \mathcal{L}_{\hat{X}} s_{0} \beta b_{i} s_{j} \\
& +6 \mathcal{L}_{\hat{X}} s_{0} \beta b_{j} s_{i}-16 \mathcal{L}_{\hat{X}} s_{0} b_{i} b_{j} s_{0}+18 \mathcal{L}_{\hat{X}} s_{i} \beta b_{j} s_{0} \\
& +12 \mathcal{L}_{\hat{X}} s_{i} \beta b_{j} t_{0}+18 \mathcal{L}_{\hat{X}} s_{j} \beta b_{i} s_{0}+12 \mathcal{L}_{\hat{X}} s_{j} \beta b_{i} t_{0} \\
& +8 \mathcal{L}_{\hat{X}} \beta b_{i} s_{j} t_{0}+8 \mathcal{L}_{\hat{X}} \beta b_{j} s_{i} t_{0}-8 \mathcal{L}_{\hat{X}} b_{i} \beta b_{j} t_{0} \\
& +18 \mathcal{L}_{\hat{X}} b_{i} \beta s_{0} s_{j}+12 \mathcal{L}_{\hat{X}} b_{i} \beta s_{j} t_{0}-8 \mathcal{L}_{\hat{X}} b_{j} \beta b_{i} t_{0} \\
& +18 \mathcal{L}_{\hat{X}} b_{j} \beta s_{0} s_{i}+12 \mathcal{L}_{\hat{X}} b_{j} \beta s_{i} t_{0}+4 \mathcal{L}_{\hat{X}} a_{i j} s_{0}^{2} \\
& -12 \mathcal{L}_{\hat{X}} q_{i j} \beta^{2}-12 \mathcal{L}_{\hat{X}} q_{j i} \beta^{2}+4 a_{i j} \mathcal{L}_{\hat{X}} q_{00}-2 e_{00 ; 0} \mathcal{L}_{\hat{X}} e_{i j} \\
& -2 e_{i 0 ; 0} \mathcal{L}_{\hat{X}} b_{j}-2 e_{i j} \mathcal{L}_{\hat{X}} e_{00 ; 0}-2 e_{j 0 ; 0} \mathcal{L}_{\hat{X}} b_{i} \\
& +4 \mathcal{L}_{\hat{X}} a_{i j} q_{00}-2 \mathcal{L}_{\hat{X}} e_{i 0 ; 0} b_{j}-10 e_{i j ; 0} \mathcal{L}_{\hat{X}} \beta+5 t_{00} q_{i j}+5 t_{00} q_{j i} \\
& +8 \mathcal{L}_{\hat{X}} e_{i j ; 0} \beta-2 \mathcal{L}_{\hat{X}} e_{j 0 ; 0} b_{i}+4 \mathcal{L}_{\hat{X}} q_{0 i} y_{j} \\
& +4 \mathcal{L}_{\hat{X}} q_{0 j} y_{i}+4 \mathcal{L}_{\hat{X}} y_{i} q_{0 j}+4 \mathcal{L}_{\hat{X}} y_{j} q_{0 i} \\
& +24 \mathcal{L}_{\hat{X}} \beta \beta b_{i} t_{j}+24 \mathcal{L}_{\hat{X}} \beta \beta b_{j} t_{i}+60 \mathcal{L}_{\hat{X}} \beta \beta s_{i} s_{j} \\
& -72 \mathcal{L}_{\hat{X}} \beta b_{i} b_{j} t_{0}-44 \mathcal{L}_{\hat{X}} \beta b_{i} s_{0} s_{j}-44 \mathcal{L}_{\hat{X}} \beta b_{j} s_{0} s_{i} \\
& -4 \mathcal{L}_{\hat{X}} e_{i 0} e_{j 0}-4 \mathcal{L}_{\hat{X}} e_{j 0} e_{i 0} \\
& A_{9}=4 \mathcal{L}_{\hat{X}} t_{0} b_{j} s_{i}-8 \mathcal{L}_{\hat{X}} t_{i} \beta b_{j}-6 \mathcal{L}_{\hat{X}} t_{j} \beta b_{i}+2 \mathcal{L}_{\hat{X}} t_{j} \beta b_{j} \\
& -6 \mathcal{L}_{\hat{X}} b_{i} \beta t_{j}+12 \mathcal{L}_{\hat{X}} \beta b_{i} t_{j}+12 \mathcal{L}_{\hat{X}} \beta b_{j} t_{i}
\end{aligned}
$$




$$
\begin{aligned}
+ & +20 \mathcal{L}_{\hat{X}} \beta s_{i} s_{j}+6 \mathcal{L}_{\hat{X}} b_{i} s_{0} s_{j}+4 \mathcal{L}_{\hat{X}} b_{i} s_{j} t_{0}-6 \mathcal{L}_{\hat{X}} b_{j} \beta t_{i} \\
& +6 \mathcal{L}_{\hat{X}} b_{j} s_{0} s_{i}+4 \mathcal{L}_{\hat{X}} b_{j} s_{i} t_{0}+2 \mathcal{L}_{\hat{X}} s_{0} b_{i} s_{j} \\
& +2 \mathcal{L}_{\hat{X}} s_{0} b_{j} s_{i}-16 \mathcal{L}_{\hat{X}} s_{i} \beta s_{j}+6 \mathcal{L}_{\hat{X}} s_{i} b_{j} s_{0}+4 \mathcal{L}_{\hat{X}} s_{i} b_{j} t_{0} \\
& -16 \mathcal{L}_{\hat{X}} s_{j} \beta s_{i}+6 \mathcal{L}_{\hat{X}} s_{j} b_{i} s_{0}+4 \mathcal{L}_{\hat{X}} s_{j} b_{i} t_{0} \\
& +4 \mathcal{L}_{\hat{X}} t_{0} b_{i} s_{j}+2 \mathcal{L}_{\hat{X}} e_{j 0} s_{i}+2 e_{j 0} \mathcal{L}_{\hat{X}} s_{i}+4 \mathcal{L}_{\hat{X}} e_{i j} s_{0} \\
& +4 e_{i j} \mathcal{L}_{\hat{X}} s_{0}+2 \mathcal{L}_{\hat{X}} e_{i 0} s_{j}+2 e_{i 0} \mathcal{L}_{\hat{X}} s_{j}-4 e_{i j} \eta \\
& -2 e_{j 0} \eta_{i}+4 \mathcal{L}_{\hat{X}} b_{i} q_{0 j}+2 \mathcal{L}_{\hat{X}} b_{i} q_{j 0}+4 \mathcal{L}_{\hat{X}} b_{j} q_{0 i} \\
& +2 \mathcal{L}_{\hat{X}} b_{j} q_{i 0}+4 \mathcal{L}_{\hat{X}} q_{0 i} b_{j}+4 \mathcal{L}_{\hat{X}} q_{0 j} b_{i}+2 \mathcal{L}_{\hat{X}} q_{i 0} b_{j} \\
& -8 \mathcal{L}_{\hat{X}} q_{i j} \beta+2 \mathcal{L}_{\hat{X}} q_{j 0} b_{i}-8 \mathcal{L}_{\hat{X}} q j i \beta+10 \mathcal{L}_{\hat{X}} \beta q_{i j} \\
A_{10}= & +10 \mathcal{L}_{\hat{X}} \beta q_{j i}-2 e_{i 0} \eta_{j}+2 \mathcal{L}_{\hat{X}} e_{i j ; 0} \\
= & -2\left(\mathcal{L}_{\hat{X}} b_{i} t_{j}+\mathcal{L}_{\hat{X}} b_{j} t_{i}+2 \mathcal{L}_{\hat{X}} s_{i} s_{j}+2 \mathcal{L}_{\hat{X}} s_{j} s_{i}\right. \\
& \left.+\mathcal{L}_{\hat{X}} t_{i} b_{j}+\mathcal{L}_{\hat{X}} t_{j} b_{i}+\mathcal{L}_{\hat{X}} q_{i j}+\mathcal{L}_{\hat{X}} q_{j i}\right) .
\end{aligned}
$$

\section{R E F E R E N C E S}

1. H. Akbar-Zadeh, Initiation to Global Finslerian Geometry, North Holland, 2006.

2. H. Akbar-Zadeh, Champs de vecteurs projectifs sur le fibré unitaire, J. Math. Pures Appl. 65 (1986) 47-79.

3. E. Beltrami, Resoluzione del problema: riportari $i$ punti di una superficie sopra un piano in modo che le linee geodetische vengano rappresentante da linee rette, Ann. Mat., 1(7) (1865) 185-204.

4. B. Bidabad and M. SePasi, On a Projectively Invariant Pseudo-Distance in Finsler Geometry, International Journal Of Geometric Methods In Modern Physics, 12(2015), $1-12$.

5. B. Chen and L. ZhaO, A note on Randers metrics of scalar flag curvature, Canad. Math. Bull., 55(2012), 474-486.

6. X. Cheng and Z. Shen, Finsler Geometry, An Approach via Randers Space, Science Press, Beijing (2012).

7. V. S. Matveev, On projective equivalence andpointwise projective relation of Randers metrics, Int. J. Math. 23(9)(2012) 1250093 (14 pages).

8. V. S. Matveev, Proof of projective Lichnerowicz-Obata conjecture, J. Differential Geom. 75 (2007) 459-502, arXiv:math/0407337.

9. B. NAJAFi and A. TAYEBI, A new quantity in Finsler geometry, C. R. Acad. Sci. Paris, Ser. I 349 (1-2) (2011) 81-83.

10. M. RAFIE-RAD, Some new characterizations of projective Randers metrics whit constant $S$ - curvature, J. Geome. Phys., (2011), 7 pages.

11. M. RAfIE-RAD, Special projective Lichnrowicz-Obata theorem for Randers spaces, C. R. Acad. Sci. Paris, Ser. I 351 (2013), 927-930.

12. M. RAFIE-RAD, B. ReZAeI, On the projective algebra of Randers metrics of constant flag curvature SIGMA, 7 (2011) 085, 12 pages 
13. A. ShIRAFKAn and M. RAFIE-RAD, On the C-projective vector fields on Randers spaces, arXiv:1811.02181v1.

14. Z. SHen, On Some Non-Riemannian Quantities in Finsler Geometry, Canad. Math. Bull. 56(1) (2013), 184-193.

15. A. TAYEBi and T. TABATABAeifar, Unicorn metrics with almost vanishing $\mathbf{H}$ - and $\Xi$ curvatures, Turkish. J. Math, 41 (2017), 998-1008.

16. A. TAyebi and M. RazGordani, On H-curvature of $(\alpha, \beta)$-metrics, Turkish J. Math, 2020, DOI:10.3906/mat-1805-130.

Tayebeh Tabatabaeifar and Behzad Najafi

Faculty of Mathematical Sciences

Department of Mathematics and Computer Sciences

Amirkabir University (Tehran Polytechnic)

t.tabatabaeifar@aut.ac.ir

behzad.najafi@aut.ac.ir

Mehdi Rafie-Rad

Department of Mathematics

Faculty of Mathematical Sciences

University of Mazandaran

Babolsar, Iran

rafie-rad@umz.ac.ir 